\title{
HUMAN MEIOSIS II. CHROMOSOME PAIRING AND RECOMBINATION NODULES IN HUMAN SPERMATOCYTES
}

by

\author{
SøREN W. RASMUSSEN and PREBEN B. HOLM
}

Department of Physiology, Carlsberg Laboratory

Gamle Carlsberg Vej 10, DK-2500 Copenhagen Valby

Keywords: Synaptonemal complex, crossing over, chromosome breaks, three dimensional reconstruction, interlocking

\begin{abstract}
Meiosis in human spermatocytes has been analyzed by three dimensional reconstructions of 4 leptotene, 4 earlymid zygotene, 10 late zygotene, and 21 early pachytene nuclei. At leptotene, a lateral component is organized along each chromosome and the telomeres attach to the nuclear envelope. At early zygotene, the attachment sites aggregate and a chromosome bouquet is formed. Pairing and synaptonemal complex formation are initiated from the telomeres by binding of precursor material for the central region to the lateral components of the aligned homologues. In the 10 late zygotene nuclei, on the average $72 \%$ of the autosomal complement had been paired. Synaptonemal complex formation is in most cases initiated from both ends of the homologues and only in 5 cases was initiation of complex formation interstitial. Pairing of the short arms of the acrocentric bivalents and the $\mathrm{X}$ and $\mathrm{Y}$ chromosomes is delayed compared to the remainder of the genome. Irregularities such as interlockings and breaks of the lateral components of chromosomes or breaks of the synaptonemal complexes of bivalents are observed in 8 out of the 10 nuclei. Most of the breaks appeared to be the result of a resolution of interlockings. At early pachytene, all bivalents are fully paired the only exception being the secondary constrictions on bivalents 1 and 9 which frequently remain unpaired. In all nuclei, a short stretch of synaptonemal complex is present between the $X$ and $Y$ chromosomes. Only two interlockings and three breaks of lateral components of chromosomes were found at this stage. Recombination nodules are present in or at the central region of the synaptonemal complex from early zygotene and evidence has been obtained in favor of an attachment of nodules to precursor material for the central region at the pairing fork. Nodules can, however, also attach to a fully formed synaptonemal complex. At late zygotene, an average number of 101 nodules per nucleus is present. Assuming that all regions of the complex have equal probabilities of receiving a nodule about 144 nodules are expected to be present at the moment of complete pairing. At early pachytene, the mean number of nodules is 75 . Generally, nodules appear to be distributed evenly along the bivalent arms. Nodules are present, however, in excess in the telomere regions and in the $\mathrm{XY}$ bivalent while the centromere regions, the secondary constrictions, and the short arms of the acrocentric bivalents are relatively depleted of nodules. Measurements of the distances between adjacent nodules and a comparison with a theoretical distribution of such distances assuming a random positioning of nodules have demonstrated that at late zygotene nodules are more frequently clustered than would be expected if they were distributed independently. At early pachytene, the reverse pattern is observed.
\end{abstract}




\section{INTRODUCTION}

A regular disjunction of homologous chromosomes during the meiotic division is a fundamental prerequisite for sexual reproduction in eukaryotes. Nondisjunction is usually fatal for the gamete, zygote or embryo. In cases of viable progeny it always leads to a reduction in viability. It is, therefore, not surprising that mechanisms have evolved to ensure regular disjoining of bivalents. With the exception of the Drosophila male (and probably other Diptera males as well) wich lack synaptonemal complexes, the regular disjunction of homologues appears to be closely connected with the presence of synaptonemal complexes. Although alignment of homologous chromosomes prior to meiosis (e.g., at the last premeiotic mitosis) has been proposed by several authors $(6,7,29,30$, 43) also for organisms lacking somatic pairing, the majority of data support the concept that chromosome pairing and synaptonemal complex formation are intimately related events at meiotic prophase $(13,35,51)$.

In organisms with achiasmatic meiosis and/or lacking crossing over the entire synaptonemal complex complement is retained until the separation of the homologous chromosomes at anaphase I $(10,41)$. In most organisms, however, the bulk of synaptonemal complexes are shed from the bivalents after pachytene leaving only shorter fragments behind $(14,15,21,27,49,53)$. It is generally believed that these retained fragments are the ultrastructural counterparts of sites where crossing over has occurred - the chiasmata - and that the fragments stabilize the crossovers, thereby holding the two homologues of each bivalent together. Crossing over thus serves two major purposes: it provides for reassortment of linked genes but also ensures the retention of a proper co-orientation of homologous chromosomes from diplotene to anaphase $I$. The mechanisms which regulate crossing over are accordingly of critical importance for both events. It is a common observation that crossovers and chiasmata are not distributed at random among the bivalents, there being a much lower frequency of bivalents without exchanges or with multiple exchanges than would be expected if crossovers and chiasmata were distributed at random. In addition, crossing over is not distributed entirely at random along the individual bivalents. Crossing over seldom occurs in the centromeric heterochromatin. Within the arms the distribution of crossovers, with respect to each other, exhibits positive interference. The observation that nonreciprocal events, as measured by gene conversion without exchange of flanking markers, occur independently of reciprocal events is intriguing, since a unitary mechanism probably accounts for both types of intrachromosomal recombination (cf. 18, 34).

The discovery of dense nodes in (or associated with) the central region of the synaptonemal complex (cf. 11, 51) and the proposal by CARPENTER (4) that the nodes may be involved in the recombination process, have provided an incitement to study the relationship between these "recombination nodules" (4) and chromosome pairing, crossing over, gene conversion, chiasmata, and disjunction.

The analyses of the number and the distribution of recombination nodules on individual bivalents have supported the proposed involvement of the nodules in recombination. In Drosophila melanogaster, the number and the distribution of recombination nodules resemble the number and the distribution of crossovers ( $a$ possible role of the nodules in gene conversion was not considered) (4). Similar agreement between distribution of crossing over or chiasmata and recombination nodules has been reported in yeast (1), Sordaria (53), and Neurospora $(11,15)$.

The present report describes in detail the morphology of human spermatocyte nuclei from leptotene to the end of early pachytene and focuses upon the pairing behaviour of the homologous chromosomes, i.e., how the homologues during zygotene become aligned into pairs, yielding at pachytene a highly regular pairing with continuous synaptonemal complexes from telomere to telomere. It also describes a mechanism whereby interlockings can become untangled. Finally, the number and distribution of recombination nodules are analyzed from early zygotene through early pachytene and are compared with theoretical distributions based on the assumption that recombination nodules become associated at random 
with the central region of the synaptonemal complex.

\section{MATERIALS AND METHODS}

\subsection{Materials}

Testicular biopsies were obtained through the courtesy of Dr. J. LINDENBERG, Dept. of Surgery K, Frederiksberg Hospital, Copenhagen. The material consists of biopsies from three normal fertile men undergoing vasectomy. A detailed description of the mid-late pachytene stage in the spermatocytes of these men has been presented in a previous publication (cases 3-5, 20). In addition, a testicular biopsy from a man carrying a balanced translocation was analyzed. This biopsy was obtained through the courtesy of Dr. P. FOGH ANDERSEN, Diakonisse Stiftelsens Hospital, Copenhagen. The translocation has been analyzed cytologically and designated as $46, X Y, t(5 ; 22)$, (p14; p12) by NiEBUHR (38, case 4). The meiotic behaviour of the translocation in early and mid pachytene is presented in a separate publication (22). Of the early pachytene nuclei reconstructed those with the numbers 39.41 and 47-50 are from the translocation heterozygote, as are the four leptotene nuclei. All zygotene nuclei are from case 5 . The early pachytene nuclei with the numbers 54-61 are from case 3 , numbers 43-44 from case 4 , and numbers $62-65$ from case 5 . In the analysis of the XY bivalents of early pachytene (Table VIII) a reconstruction was included from a partially analyzed nucleus of the translocation heterozygote otherwise not considered.

\subsection{Electron microscopy}

Immediately after removal, the testicular biopsies were placed in a $4 \%$ phosphate buffered $(0.06 \mathrm{M}, \mathrm{pH} 7.2)$ glutaraldehyde solution with a purification index of about 0.2 , cut in smaller pieces, transferred to fresh glutaraldehyde and fixed for $3 \frac{1}{2}$ hours at room temperature. After two rinses in phosphate buffer, the tissue was postfixed in $2 \%$ osmiumtetroxide in phosphate buffer at room temperature for two hours, washed twice in buffer, 4-5 times in distilled water, and left overnight in distilled water at $4^{\circ} \mathrm{C}$. The biopsies were then fixed/stained in a
$2 \%$ aqueous uranyl acetate solution at $60^{\circ} \mathrm{C}$ for 3 hours. After cooling to room temperature, the tissue was dehydrated in a graded alcohol series, embedded in SPURR's low viscosity resin and polymerized for 24 hours at $70^{\circ} \mathrm{C}$.

Thick sections (about $1 \mu \mathrm{m}$ ) of the seminiferous tubules were examined in the light microscope for a preliminary identification of meiotic stages. Nuclei in the relevant stages of meiotic prophase were serially sectioned on a Reichert Om U3 ultramicrotome equipped with a diamond knife. The serial sections were picked up on single slot grids without formvar film and thereafter transferred onto grids with supporting film, using a micromanipulator for maneuvering the grids during the transfer process. Sections were stained in a $5 \%$ aqueous uranyl acetate solution for $30 \mathrm{~min}$ at $50^{\circ} \mathrm{C}$, rinsed, and poststained in lead citrate for $20 \mathrm{~min}$. The staining was carried out in an automatic staining apparatus. Sections were examined, and selected nuclei were photographed in a Siemens Elmiscope 102 at a primary magnification of 5,000 times.

\subsection{Three dimensional reconstruction}

Three dimensional reconstructions were performed in the following way: The synaptonemal complex segments from groups of ten sections were traced onto transparent plastic film. After having encompassed the whole nucleus, the tracings of all synaptonemal complex segments of each bivalent were redrawn on a new sheet of plastic film together with the relevant section numbers. The projected lengths were measured on a Hewlett Packard digitizer (HP 9864A) and the absolute length in $\mu \mathrm{m}$ automatically calculated with the aid of a Hewlett Packard calculator (HP 9825A) using the Pythagorean Theorem $a^{2}+b^{2}=c^{2}$ (where $a$ is the projected length, $b$ the section thickness, and $c$ the absolute length). The estimated section thickness was $0.08 \mu \mathrm{m}$. After having measured all bivalents in a nucleus, the autosomes were automatically classified by the computer according to length and centromere indices following the criteria previously described (20). Finally, an idiogram for each nucleus was automatically drawn by a HP 9872A four colour plotter. 


\subsection{Calculations}

In order to analyze the distribution of recombination nodules on the different bivalent arms, the length of each arm of a bivalent was adjusted to the mean length of that particular bivalent arm and the relative position of the recombination nodule(s) was determined for the normalized arm. These normalized bivalent arms were then divided into segments of 0.67 $\mu \mathrm{m}$. The mean number of nodules per segment of each bivalent arm was calculated and used to illustrate the distribution of nodules along the synaptonemal complex. The theoretical distributions of nodule-nodule, nodule-telomere, and nodule-centromere distances were constructed with the aid of a Honeywell Bull $\mathrm{H}$ 6000 computer.

\section{RESULTS}

\subsection{Leptotene}

The leptotene stage of meiosis is at the ultrastructural level characterized by the organization of a lateral component along the two sister chromatids of the individual chromosome (Figure 1). The fully organized lateral component is an electron dense rod, $30 \mathrm{~nm}$ in diameter with a filamentous substructure and with a round to ovoid shape in cross section. As shown in Figure 1, the chromatin is evenly distributed around the lateral components at this stage. In the four serially sectioned nuclei 76 , 82,88 , and 83 of the 92 telomeres are attached to the nuclear envelope through a conical thickening of the lateral component. In some cases the acrocentric chromosomes can be identified by their association with nucleoli. This association is mediated by a body of low electron density which probably marks the position of the nucleolus organizer region. Nucleoli associated with the lateral components are frequently present in the middle of the nucleus suggesting that not all the short arms of the acrocentric chromosomes are attached to the nuclear envelope despite their apparently completed lateral components (Figure 2). In the middle regions of all chromosomes, the lateral components are frequently either diffuse or apparently lacking over larger distances making reconstructions of entire chromosomes ambiguous or impossible. Although the majority of the chromosomes are present as univalents, chromosome pairing and synaptonemal complex formation have started, thus demonstrating that completion of lateral component organization is not a prerequisite for synaptonemal complex formation. The attachment sites of the telomeres are distributed over most of the inner membrane of the nuclear envelope and the distinct chromosome bouquet characteristic of the zygotene stage (cf. Figures 4 and 7) has not yet formed. Outside the nucleus two centrioles with their procentrioles are present surrounded by Golgi apparatuses. The ultrastructure of the centrioles and the size of the procentrioles are indistinguishable from those seen at the zygotene and pachytene stages.

\subsection{Early-mid zygotene}

Two nuclei in early zygotene and two in mid zygotene were serially sectioned and reconstructed (Figure 3 and 4). The establishment of the temporal relationship between these nuclei was based on the degree of pairing of the chromosome complement. In the two early zygotene nuclei, the length of the region paired with a synaptonemal complex was $44.1 \mu \mathrm{m}$ and in the two mid zygotene nuclei $145.1 \mu \mathrm{m}$ (Table VI). This corresponds to $19 \%$ and $62 \%$ of the complement length recorded at late zygotene (see section 3.3.3., Table VI). The lateral components of the unpaired chromosome regions

Figure 1. Two consecutive sections through a nucleus at the transition between leptotene and zygotene. The nucleus has a circular outline with a group of Golgi apparatuses (GA) lying close to the outer nuclear membrane. The chromatin appears evenly distributed in the karyoplasm. Several stretches of lateral components (LC) in cross and longitudinal sections are present in the micrographs. Telomeres attached to the nuclear envelope are denoted by arrows.

$(\mathrm{Bar}=2 \mu \mathrm{m})$ 

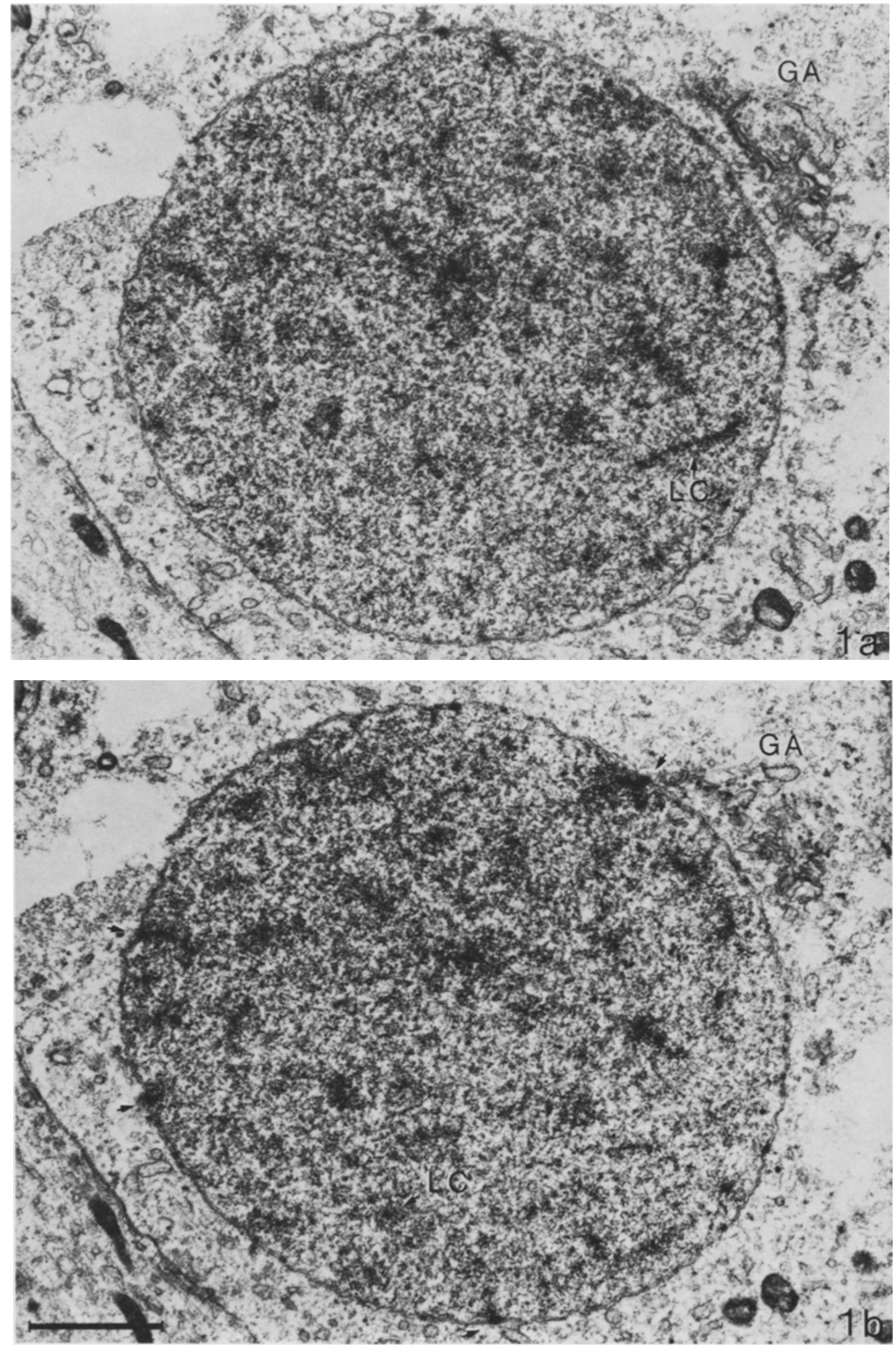


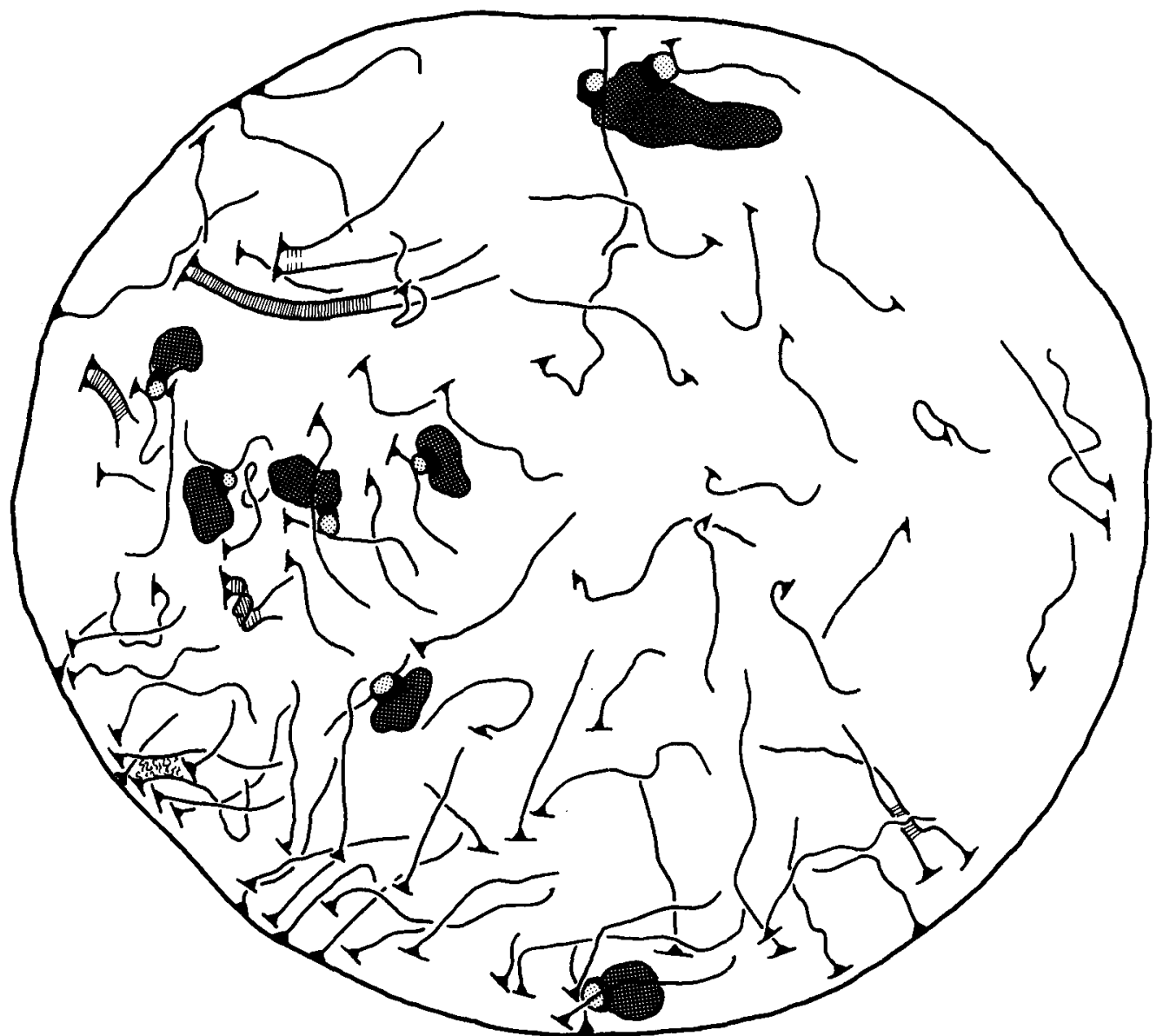

Figure 2. A partial reconstruction of a human spermatocyte nucleus at the transition between leptotene and zygotene (nucleus number 32). Most of the chromosomes are not aligned with their homologue. Chromosome pairing with synaptonemal complex formation has started in five chromosome arms, In one of these arms, the central region has not yet attained its final organization. Synaptonemal complex formation between two homologous lateral components starts with one exception from the nuclear envelope. Precursor material for the central region is frequently present between the grossly aligned lateral components of two homologous chromosomes (see also Figure 5). Only 82 telomeres are anchored at the inner membrane of the nuclear envelope and a weak polarization of the telomere attachment sites can be seen. Eight of the ten nucleolus organizing chromosomes have been identified in this partial reconstruction. In two of these the lateral components of the short arms are not attached to the nuclear envelope.

were discontinuous in all four nuclei making reconstructions of the entire complement impossible. The majority of the telomeres was attached to the inner membrane of the nuclear envelope ( 85 and 90 in the two early zygotene nuclei and 86 and 91 in the mid zygotene nuclei) within a limited area thus giving rise to a prominent chromosome bouquet (Figure 4).

The individual phases of chromosome pairing and synaptonemal complex organization are clearly illustrated by these nuclei. Prior to the synaptonemal complex formation, homologous telomere regions are grossly aligned and aggregates of fibrillar material assumed to be precursors of the central region accumulate between the two homologues (Figures 4 and 5). Material with a similar ultrastructure frequently binds to unpaired lateral components including those of the differential segment of the $X$ chromosome. Following the alignment of 


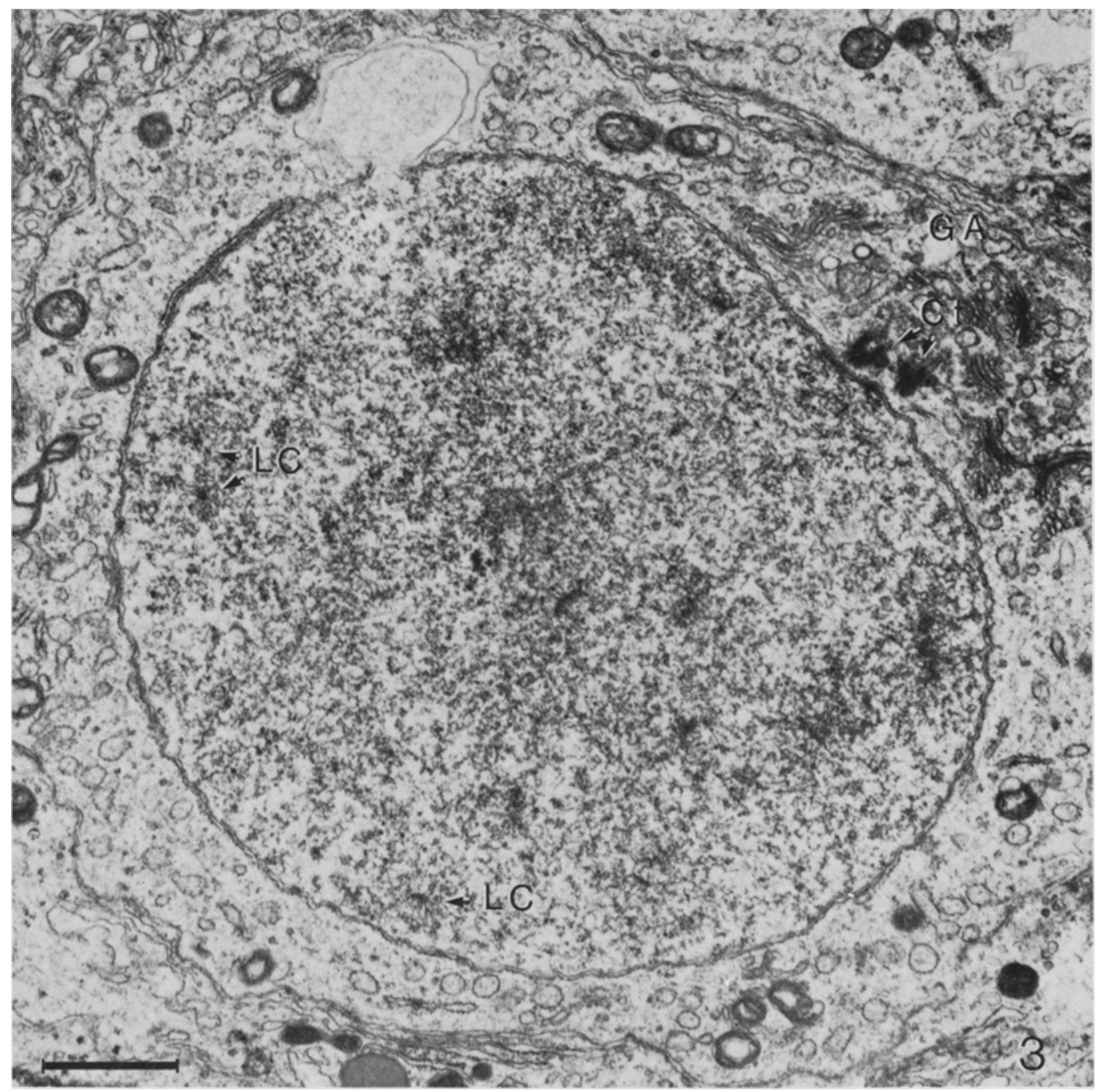

Figure 3. Survey micrograph of an early zygotene nucleus. The nucleus is kidney-shaped with the flattened side facing an aggregate of Golgi apparatuses (GA). The two centrioles (Ct) and their procentrioles are present within this aggregate. Several unpaired lateral components (LC) are seen in cross section. An evagination of the nuclear envelope is present in the upper half of the nucleus.

$($ Bar $=2 \mu \mathrm{m})$

homologous lateral components, the central region with its medially positioned central component is organized either along only one of the two lateral components or possibly along both lateral components simultaneously. In the first case organization of the synaptonemal complex is completed by the association of the second lateral component to the free face of the central region. In the middle region of the chromo- somes, the organization of the lateral components appears to coincide with the intimate pairing and synaptonemal complex formation.

In all bivalents pairing and synaptonemal complex formation are initiated from the nuclear envelope before a complete gross alignment in the middle regions of the homologous has taken place. Within a nucleus chromosome pairing is highly asynchronous as illustrated in 


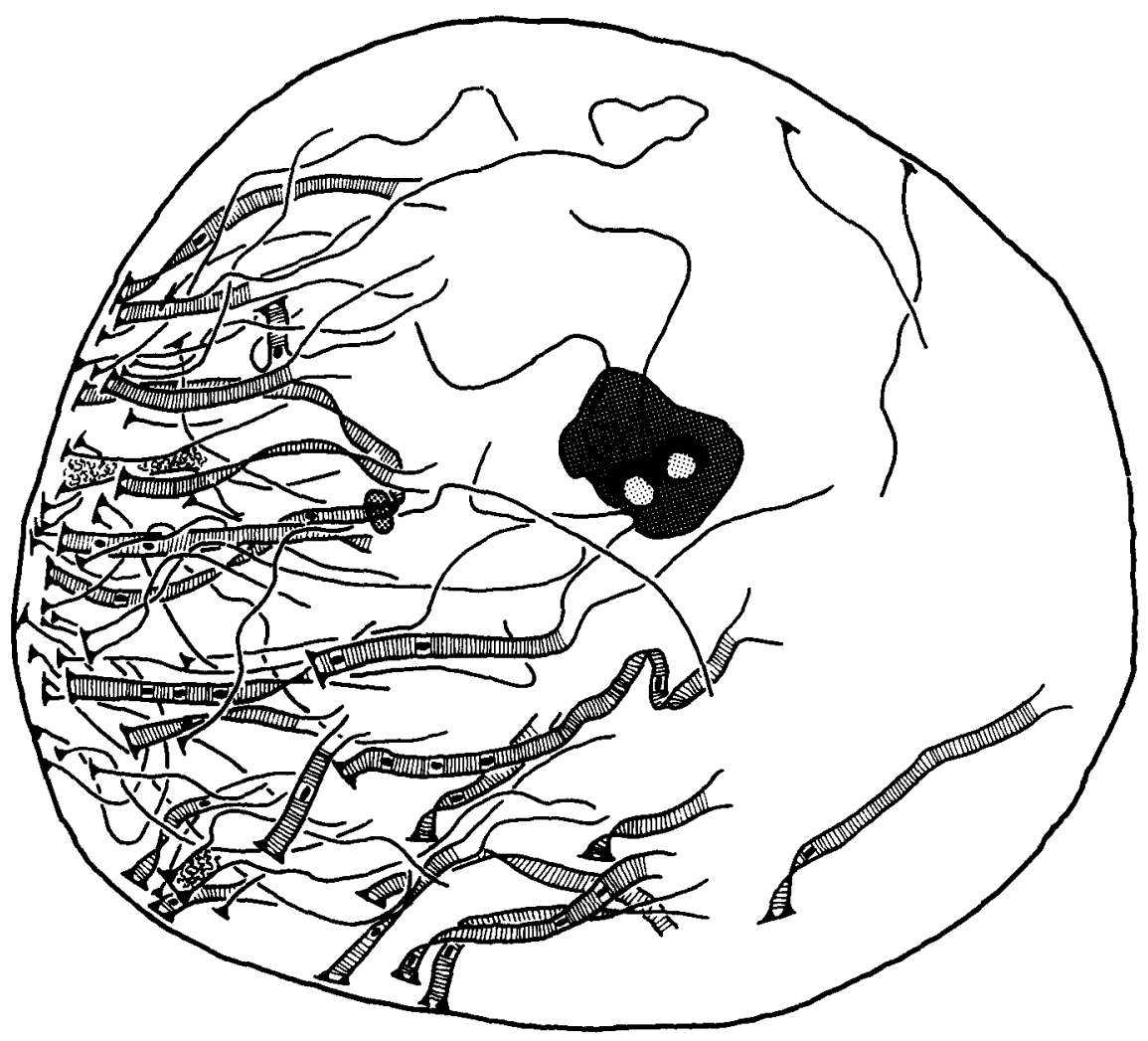

Figure 4. A reconstruction of an early zygotene nucleus. The majority of the chromosomes are arranged in a bouquet with their telomeres attached within a limited region of the nuclear envelope. The pairing behaviour is highly asynchronous. Two homologous chromosomes are completely paired with a synaptonemal complex from telomere to telomere. (The cross-hatched area denotes the centromeric heterochromatin.) Several pairs of chromosomes have synapsed over a smaller part of their lenght with synaptonemal complex formation progressing from the nuclear envelope. Twenty-five recombination nodules, all associated with the central region of the synaptonemal complex, are present in this nucleus. Most of the unpaired chromosomes have their telomeres close together on the nuclear envelope and precursor material for the central region is present between the homologues. Of the 92 telomeres 85 are attached to the nuclear envelope. Note the free telomeres of two nucleolus organizing chromosomes in the middle of the nucleus. The other nucleoli found in this nucleus are not included in the reconstruction for the sake of clarity.

Figure 4, where only one bivalent is fully paired with a synaptonemal complex extending from telomere to telomere, while the remaining chromosomes are either completely unpaired or only partly paired. The completely paired bivalent possesses one region of condensed chromatin. The present analysis of late zygotene and early pachytene nuclei as well as previous investigations (20) have demonstrated that these regions of precociously condensed chromatin are the counterparts of the centromeric heterochromatin of mitotic chromo- somes (39) and diakinesis bivalents (24). In the majority of the partly paired bivalents electron dense nodes, recombination nodules, are associated with the central region of the synaptonemal complex (see section 3,7. for a description of recombination nodules during early and mid zygotene).

\subsection{Late zygotene \\ 3.3.1. General observations}

Ten nuclei in late zygotene, all from the same male $(20$, case number 5$)$ were serially section- 

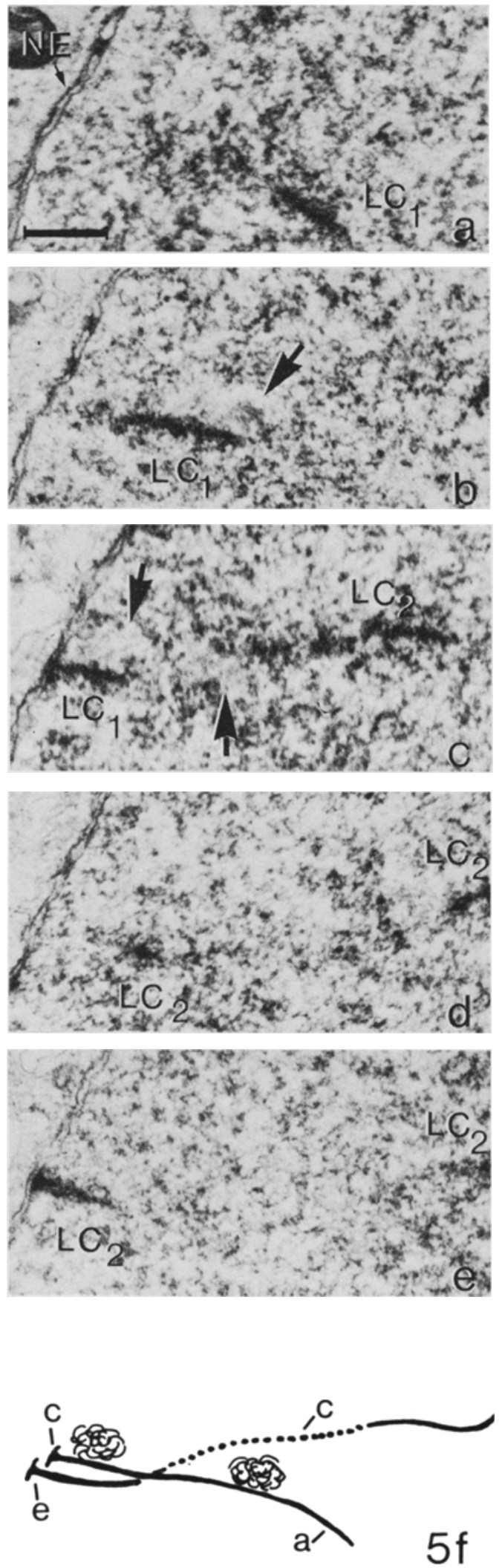

ed and completely reconstructed. The stage is characterized by the late phases of pairing and synaptonemal complex formation. The ultrastructure of a late zygotene nucleus is illustrated in Figure 6. The lateral components of the unpaired chromosome regions are distinct and continuous. Each chromosome contains one small region of centromeric heterochromatin intimately associated with the lateral component. In the completely paired bivalents, chromatin fibers penetrate the central region of the complex in these regions in contrast to mid and late pachytene bivalents, where the central region is devoid of chromatin in the centromere region (20).

At late zygotene, the telomeres of all chromosomes are attached to the nuclear envelope, most of them within the region facing the centrioles and the Golgi apparatuses, thereby forming the chromosome bouquet (Figure 7). The increase in the amount of synaptonemal complex from early to late zygotene is accompanied by an increase in the number of recombination nodules (see section 3.7. and Table VI).

Large in- and evaginations of one or both membranes of the nuclear envelope are frequently present and form electron translucent vacuole-like areas in the cytoplasm and the karyoplasm.

\subsubsection{The late zygotene karyotype}

In previous investigations in mid-late pachytene nuclei (20), it was demonstrated that 14 of the 22 autosomal bivalents could be identified and the remaining 8 bivalents assigned to a major group on the basis of chromosome mar-

Figure 5. Five consecutive sections illustrating the alignment of two homologous chromosomes in an early zygotene nucleus. A reconstruction of these five sections is shown in Figure $5 f$. In Figures $5 b$ and $c$ filamentous precursor material for the central region is present (arrows). One of the homologues has not yet organized a continuous lateral component (Figures $5 c$ and $5 f$ ).

$\mathrm{NE}$, nuclear envelope

$(\mathrm{Bar}=0.5 \mu \mathrm{m})$ 

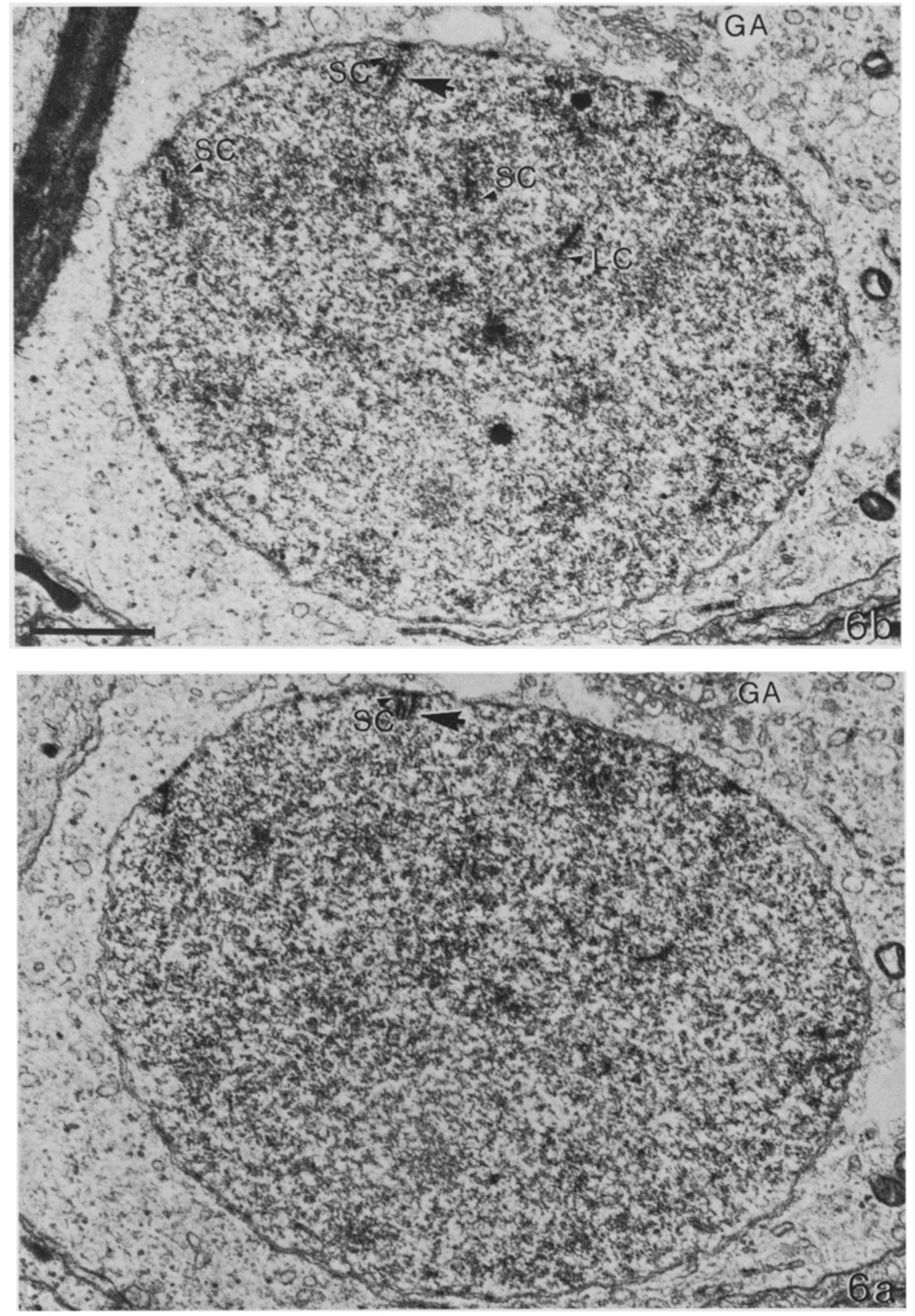

Figure 6. Two consecutive sections through a late zygotene nucleus. The nucleus is kidney-shaped with the flattened side facing an aggregate of Golgi apparatuses (GA). The nucleus contains several stretches of synaptonemal complex (SC) and unpaired lateral components (LC). Note the two recombination nodules close to the telomere of one bivalent (arrows). 


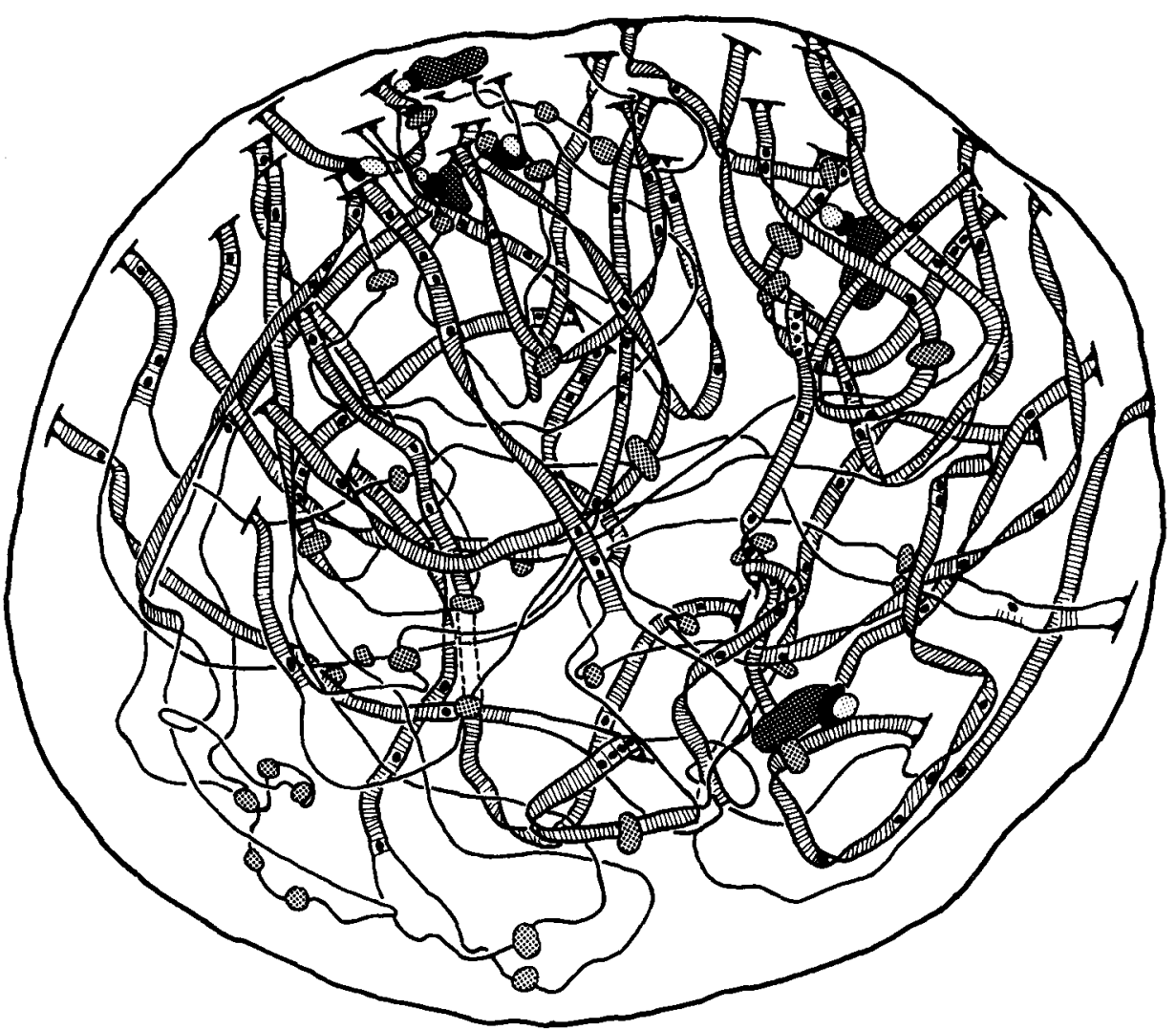

Figure 7. A complete reconstruction of a late zygotene nucleus (nucleus number 67). Most of the chromosome complement $(71 \%$, Table II) is paired with synaptonemal complexes. Note the chromosome bouquet and the large number of recombination nodules (113, TableII). Centromeric heterochromatin and knobs are shown with an intermediate grade of hatching, nucleoli with a dark hatching and the filamentous body at the nucleolus organizing region with a light hatching. The nucleolus is connected to the filamentous body through material of very high electron density (black). See also Figure 13.

kers and measurements of length and centromere indices. The so-called secondary constrictions distal to the centromeric heterochromatin in the long arms served as markers on bivalents 1,9 , and 16 . The criteria used in the classification by length and centromere indices were based on the data by HuLTÉN (24) for diakinesis bivalents. The analysis of a translocation between chromosomes 5 and 22 (22) has, however, demonstrated that the criteria previously used to distinguish between bivalents 4 and 5 were incorrect. The two bivalents cannot be separated by length, whereas in most cases bivalent 5 possesses a lower centromere index than bivalent 4.
The two unpaired lateral components of a partially paired bivalent are generally of the same length, the variation seldom exceeding $10 \%$ of their length. This difference is considered to be insignificant as it equals the maximum error of the method of reconstruction. Using the mean lateral component length of unpaired regions as an estimate of the corresponding length of the synaptonemal complex, the partially paired bivalents in late zygotene can be classified to the same extent as in mid-late pachytene by synaptonemal complex length and centromere indices. Furthermore, the secondary constriction on bivalents 1,9 , and 16 can be indentified at this stage by their indistinct un- 
paired lateral components. Bivalents 1-5, 9, 12 and 16-22 can thus be identified unequivocally while the classification of bivalents 6-8, 10-11, and 13-15 beyond an assignment to the $C$ and $D$ groups is somewhat ambiguous due to similarities in lengths and centromere indices. The mean absolute lengths, relative lengths and the centromere indices for the 22 autosomes are given in Table I.

\subsubsection{Total complement length per nucleus during zygotene}

The total length per nucleus of the autosomes is presented in Table II. The lengths of the partially paired bivalents are calculated by adding the synaptonemal complex length of the paired region and the mean lateral component length of the unpaired region. The lengths of the complement vary from $191.9 \mu \mathrm{m}$ to $271.3 \mu \mathrm{m}$ with a mean value of $235 \pm 26 \mu \mathrm{m}$. The ten nuclei listed in Table II are in a similar stage of pairing as judged by the percent of the complement paired with a synaptonemal complex, the values ranging from $67 \%$ pairing to $80 \%$ pairing with a mean value of $72 \pm 5 \%$.

\subsubsection{The $X$ and $Y$ chromosomes}

At late zygotene, the lateral components of the $\mathrm{X}$ and $\mathrm{Y}$ chromosomes are indistinguishable in ultrastructure from unpaired lateral com-

\section{Table I}

Mean values of absolute length, relative length, centromere index, and number of recombination nodules for the 22 bivalents of 10 zygotene nuclei.

\begin{tabular}{|c|c|c|c|c|c|c|c|c|c|}
\hline $\begin{array}{l}\text { Bivalent } \\
\text { number }\end{array}$ & $\begin{array}{l}\text { Absolute } \\
\text { length }(\mu \mathrm{m})\end{array}$ & $\begin{array}{l}\text { Standard } \\
\text { deviation }\end{array}$ & $\begin{array}{l}\text { Relative } \\
\text { length(\%) }\end{array}$ & $\begin{array}{l}\text { Standard } \\
\text { deviation }\end{array}$ & $\begin{array}{l}\text { Cen- } \\
\text { tromere } \\
\text { index }\end{array}$ & $\begin{array}{l}\text { Standard } \\
\text { deviation }\end{array}$ & $\begin{array}{l}\text { Number } \\
\text { of } \\
\text { nodules }\end{array}$ & $\begin{array}{l}\text { Standard } \\
\text { deviation }\end{array}$ & $\begin{array}{l}\text { Expected } \\
\text { number of } \\
\text { nodules }^{\text {a) }}\end{array}$ \\
\hline 1 & 19.2 & 2.1 & 8.2 & 2.5 & 51 & 5 & 6.2 & 2.5 & 11.9 \\
\hline 2 & 17.4 & 1.9 & 7.5 & 0.5 & 40 & 4 & 4.7 & 2.3 & 9.8 \\
\hline 3 & 15.4 & 2.1 & 6.6 & 0.6 & 45 & 4 & 6.5 & 4.0 & 9.8 \\
\hline 4 & 14.1 & 1.4 & 6.0 & 2.3 & 36 & 5 & 5.3 & 1.6 & 8.4 \\
\hline 5 & 13.7 & 1.6 & 5.8 & 0.1 & 30 & 4 & 5.3 & 3.5 & 9.3 \\
\hline 6 & 13.2 & 1.5 & 5.5 & 0.4 & 31 & 7 & 6.6 & 2.2 & 11.4 \\
\hline 7 & 12.6 & 1.4 & 5.4 & 0.2 & 37 & 7 & 3.6 & 1.4 & 5.1 \\
\hline 8 & 11.1 & 1.3 & 4.7 & 1.9 & 36 & 4 & 6.9 & 3.1 & 8.4 \\
\hline 9 & 11.8 & 1.0 & 5.1 & 0.3 & 37 & 3 & 5.0 & 2.8 & 6.3 \\
\hline 10 & 11.8 & 1.3 & 5.0 & 0.3 & 34 & 5 & 5.1 & 2.3 & 5.6 \\
\hline 11 & 10.5 & 1.7 & 4.5 & 0.4 & 38 & 6 & 5.3 & 1.9 & 6.0 \\
\hline 12 & 11.4 & 1.4 & 4.9 & 0.3 & 28 & 3 & 4.9 & 2.3 & $5: 8$ \\
\hline 13 & 9.9 & 2.1 & 4.2 & 0.6 & 14 & 4 & 4.2 & 2.5 & 5.3 \\
\hline 14 & 8.4 & 1.2 & 3.6 & 0.3 & 14 & 5 & 3.2 & 2.1 & 4.2 \\
\hline 15 & 8.0 & 1.3 & 3.4 & 0.2 & 15 & 3 & 3.6 & 3.1 & 5.7 \\
\hline 16 & 8.4 & 1.0 & 3.6 & 0.3 & 4.3 & 3 & 3.8 & 1.3 & 4.4 \\
\hline 17 & 8.5 & 1.1 & 3.6 & 0.3 & 33 & 3 & 5.4 & 2.5 & 5.7 \\
\hline 18 & 6.3 & 0.7 & 2.6 & 0.2 & 26 & 3 & 4.6 & 1.6 & 4.6 \\
\hline 19 & 6.2 & 0.7 & 2.7 & 0.3 & 43 & 4 & 4.4 & 3.2 & 4.4 \\
\hline 20 & 8.0 & 1.1 & 3.4 & 0.3 & 43 & 4 & 4.0 & 1.6 & 4.4 \\
\hline 21 & 3.6 & 0.6 & 1.6 & 0.1 & 26 & 5 & 2.4 & 2.2 & 2.8 \\
\hline 22 & 5.4 & 1.4 & 2.3 & 0.4 & 19 & 4 & 2.7 & 1.7 & 2.9 \\
\hline
\end{tabular}

a) The expected number of nodules at completion of synapotonemal complex formation is calculated with the assumption that nodules remain in their original position and that all regions of the synaptonemal complex have equal possibilities of receiving a nodule. These values are calculated as number of nodules/fraction of pairing. 
Table II

Total complement length, percent lateral component paired into synaptonemal complex, total number of recombination nodules, and synaptonemal complex length per recombination nodule for 10 zygotene nuclei.

\begin{tabular}{cccccc}
\hline $\begin{array}{c}\text { Nucleus } \\
\text { number }\end{array}$ & $\begin{array}{c}\text { Total } \\
\text { complement } \\
\text { length }(\mu \mathrm{m}) \\
\text { a) }\end{array}$ & $\begin{array}{c}\text { Percent } \\
\text { complement } \\
\text { paired into } \\
\text { SC }\end{array}$ & $\begin{array}{c}\text { Total } \\
\text { number of } \\
\text { nodules }\end{array}$ & $\begin{array}{c}\text { SClength } \\
(\mu \mathrm{m}) \text { per } \\
\text { nodule }\end{array}$ & $\begin{array}{c}\text { Expected } \\
\text { total } \\
\text { number of } \\
\text { nodules b) }\end{array}$ \\
\hline 66 & 234.1 & 67 & 88 & 1.8 & 131 \\
67 & 267.0 & 71 & 113 & 1.7 & 159 \\
68 & 271.3 & 75 & 161 & 1.3 & 215 \\
69 & 250.8 & 71 & 113 & 1.6 & 159 \\
70 & 229.7 & 66 & 75 & 2.0 & 114 \\
71 & 214.5 & 80 & 110 & 1.6 & 138 \\
72 & 240.3 & 76 & 95 & 1.9 & 125 \\
73 & 204.9 & 69 & 107 & 1.3 & 135 \\
74 & 191.9 & 69 & 96 & 1.3 & 103 \\
75 & 242.3 & 79 & 81 & 2.4 & 144 \\
\hline Mean & 234.7 & 72 & 101 & 1.7 & 32 \\
Standard & 25.6 & 5 & 25 & & 0.3 \\
deviation & & & & & \\
\hline
\end{tabular}

a) Synaptonemal complex length plus the mean lateral component length of the unpaired segments

b) The expected number of nodules at completion of synaptonemal complex formation is calculated by dividing the total number of nodules with the fraction of pairing, see footnote to Table I.

ponents of the autosomes. The lateral component of the $\mathrm{Y}$ chromosome is associated with two heterochromatic knobs, one of which probably constitutes the centromeric heterochromatin. Up to five knobs of similar ultrastructure are present on the $\mathrm{X}$ chromosome. The nucleolus- like structures which are associated with the $\mathrm{Y}$ chromosome at early pachytene (see section 3.4.4.) have not been found at this stage. The absolute lengths of the $\mathrm{X}$ and $\mathrm{Y}$ chromosomes at late zygotene are given in Table VIII.

\subsubsection{General aspects of chromosome pairing}

With a few exceptions (see section 3.3.6.) pairing of autosomal homologous chromosomes into bivalents was highly regular. Among the 10 nuclei completely unpaired homologues were found in only two cases (nucleus number 70 , chromosome 14 ; nucleus number 75 , chromosome 15). In 23 cases pairing of the two homologues had started from only one end, 22 of these being acrocentric bivalents with unpaired short arms. Initiation of pairing in a non-telomeric segment was revealed in 5 bi- valents (cf. Figure 10b, bivalent 4; Figure 10c, bivalent 9). The remaining bivalents were either completely paired or were partially paired with pairing progressing from both telomeres. The unpaired lateral components generally diverge at various angles from the point of synaptonemal complex formation - the pairing fork and a gross alignment similar to that of the telomere regions is seldom seen (Figures $\mathbf{8 , 9}$ ).

Figures $10 \mathrm{a}-\mathrm{d}$ contain a reconstruction of the whole chromosome complement of a late zygotene nucleus and the autosomal complement of this nucleus is shown in Figure 11 as an idiogram. The histograms in Figure 12 summarize the extent of synaptonemal complex formation in the 22 autosomal chromosome pairs in the 10 late zygotene nuclei. The following general characteristics of chromosome pairing at late zygotene can be deduced from this diagram: 1) The shorter bivalents (numbers 17-20) are completely paired or nearly so at this stage; 2 ) Pairing of the short arms of the acrocentric chromosomes is incomplete, only $40 \%$ of the arms being paired with a synaptonemal complex; 3) Pairing of the homologues of bivalents $1-12$ and 

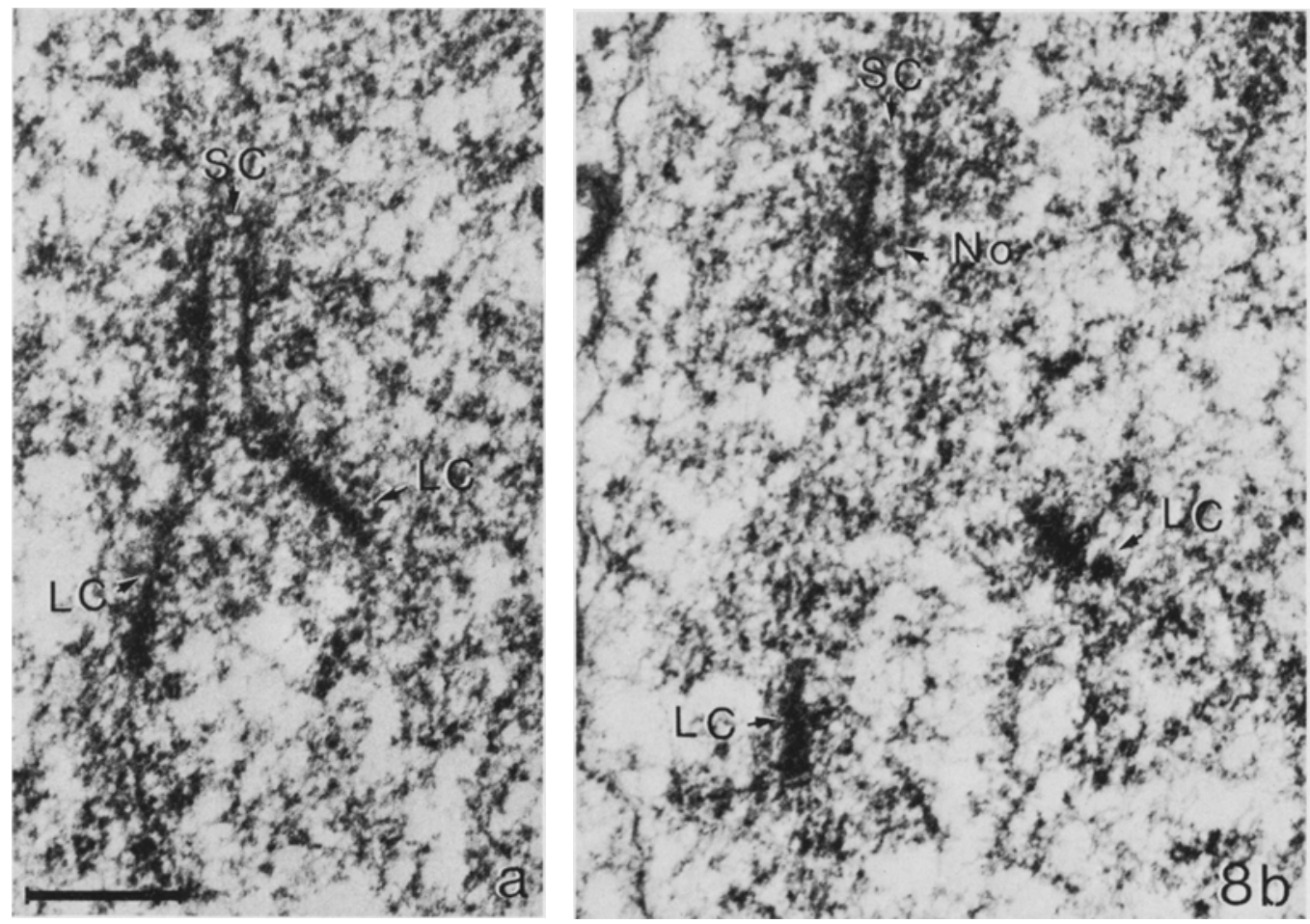

Figure 8. Two consecutive sections through a pairing fork of a late zygotene bivalent (long arm of bivalent 2, nucleus number 73). The space between the two unpaired lateral components (LC) is filled with chromatin. A recombination nodule (No) is present in Figure 8b.

$\mathrm{SC}$, synaptonemal complex

$($ Bar $=0.5 \mu \mathrm{m})$

16 is in most cases initiated from both ends and occasionally traverses the centromeric heterochromatin; 4) The secondary constrictions of bivalents 1,9 , and 16 (the segment to the left of the centromere) is in most cases unpaired; 5) The histograms for bivalents $5,7,9,10,12$, and 16 exhibit a sharp decrease in the frequency of pairing across the centromere, with maximum pairing in the short arms of these bivalents, suggesting a delay in the rate of synaptonemal complex formation in the centromeric region.

In 5 out of 10 nuclei, the $X$ and $Y$ chromosomes were unpaired although their homologous telomeres, with one exception, were attached to the nuclear envelope in the vicinity of each other (Tables VIII and IX). This indicates a delayed pairing and synaptonemal complex formation for the $\mathrm{X}$ and $\mathrm{Y}$ chromosomes.

\subsubsection{Interlockings and breaks}

The results presented in section 3.3.5. have demonstrated a highly regular pairing of most of the bivalents during zygotene. In 8 of the 10 nuclei irregularities were, however, noted either in the form of chromosome or bivalent interlockings or as breaks of the lateral components of chromosomes or the synaptonemal complex of the bivalents (Table III). Entrapment of one homologue of a partially paired bivalent between an unpaired segment of another bivalent (chromosome interlocking) was observed in three cases (Figures 10b, 13, and 14). Bivalent interlocking, i.e., the entrapment of a bivalent between the partially paired homologues of a different bivalent, was observed twice (Figures 14 and 15). One of these cases was interpreted as a double interlocking in 

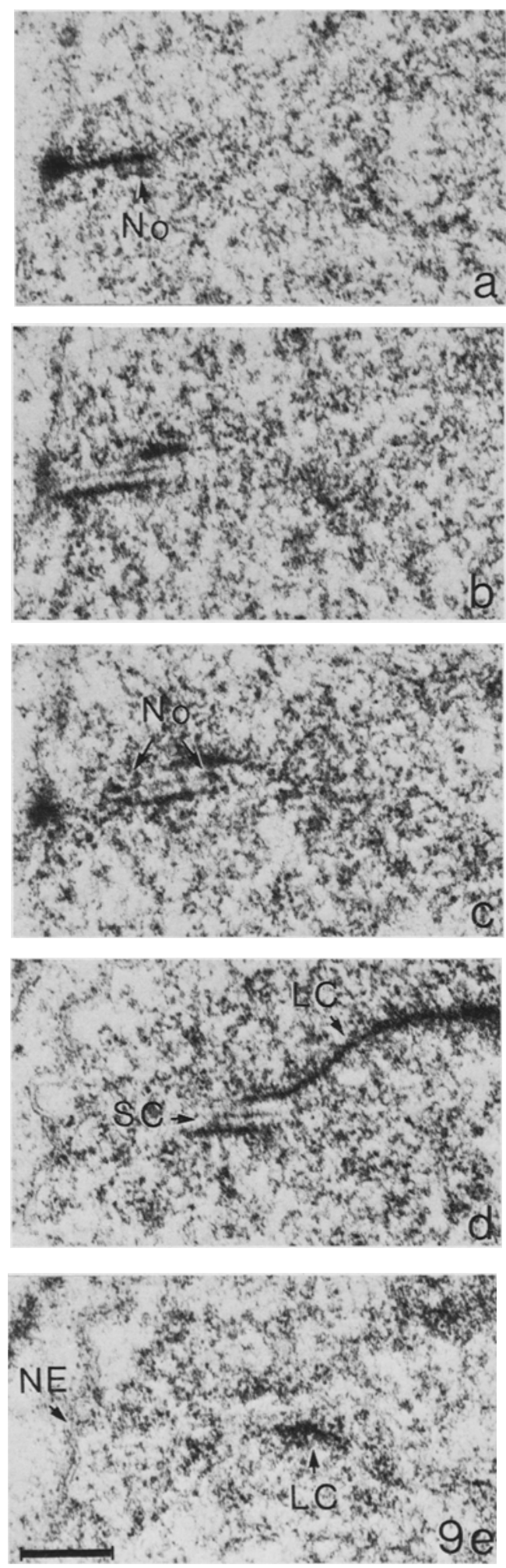

Figure 9. Five consecutive sections through a pairing fork of a late zygotene bivalent (short arm of bivalent 6 , nucleus number 66, Figure 10c). The lateral components (LC) of the synaptonemal complex (SC) are anchored at the inner membrane of the nuclear envelope (NE) (Figures 9a and 9c). Three recombination nodules (No) are attached to the central region of the complex, two in Figure $9 c$ and one in Figure 9a. The latter is less electron dense and appears to be double. The two lateral components diverging from the paired region are included in Figures $9 \mathrm{~d}$ and $\mathrm{e}$. $(B a r=0.5 \mu \mathrm{m})$

which a paired segment of bivalent 2 was entrapped between the homologues of bivalent 16 , and one homologue of bivalent 2 was interlocked between the homologues of bivalent 4 (Figure 14).

A chromosome or bivalent break is assumed to have occurred when the lateral component or the synaptonemal complex was absent within a well-defined segment, limited by two distinct end points of lateral components or synaptonemal complexes, and when the combined lengths of the two segments with continuous lateral components or synaptonemal complexes were equal to the estimated length of that particular chromosome or bivalent. Five chromosome and 2 bivalent breaks were revealed, most of them probably being the result of a resolution of interlockings. A broken chromosome, namely a subterminal break of the $\mathrm{X}$ chromosome (Figure 16), not located in the vicinity of a partially paired bivalent, was revealed only once in the 10 nuclei. The remaining 6 cases are illustrated in Figures 17-20 and 10d. Case 1: The reconstruction shown in Figure 17 is particularly suggestive of an interpretation of the break in the long arm of chromosome 13 as being the result of a resolution of a former interlocking, one of the breaks being located between the homologues of bivalent 5. Case 2: As illustrated in Figure 10d, the long arm of one homologue of bivalent 7 has probably been interlocked between the two homologues of bivalent 1 , the interlocking being resolved by a subterminal break of chromosome 7 (Figures 10d and 22). Cases 3,4: The broken end of one homologue of chromosome 14 (Figure 18) is positioned between the two homologues of bivalent 5 . The 

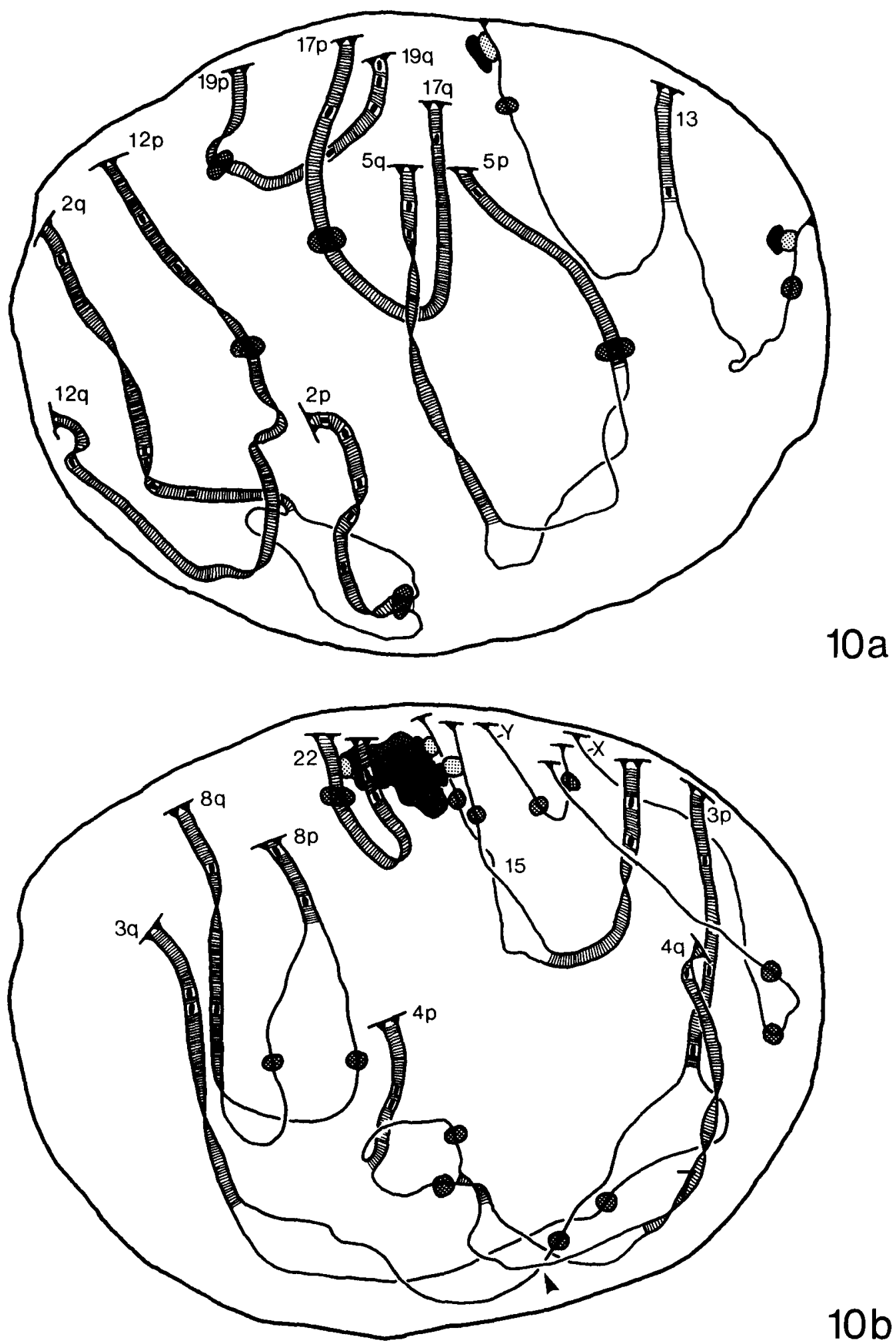

$10 b$

Figure 10. A complete reconstruction of a late zygotene nucleus (nucleus number 66). The completely or partially paired homologues (67\% paired, Table 11 ) are organized in a bouquet. The two homologues of bivalent numbers 11,12 , and 17-22 are fully paired with continuous synaptonemal complexes throughout their entire lenghts. Bivalent 16 is fully paired except for the secondary constriction next to the centromere in the long arm. The lateral components of this region are discontinuous as indicated by broken lines (Figure 10c). The secondary constrictions of bivalents 1 and 9 are likewise unpaired with discontinuous lateral components (Figures 10c and d). Synaptonemal complex formation appears to be initiated from the telomeres in the majority of the bivalents. In this nucleus only bivalents 4 and 9 have formed a synaptonemal complex internally in the long arms proximal to the centromere (Figures $10 \mathrm{~b}$ and $\mathrm{c}$ ). In bivalents $2,5,7,9,10$ and 16 the short arms are paired to or just beyond the 

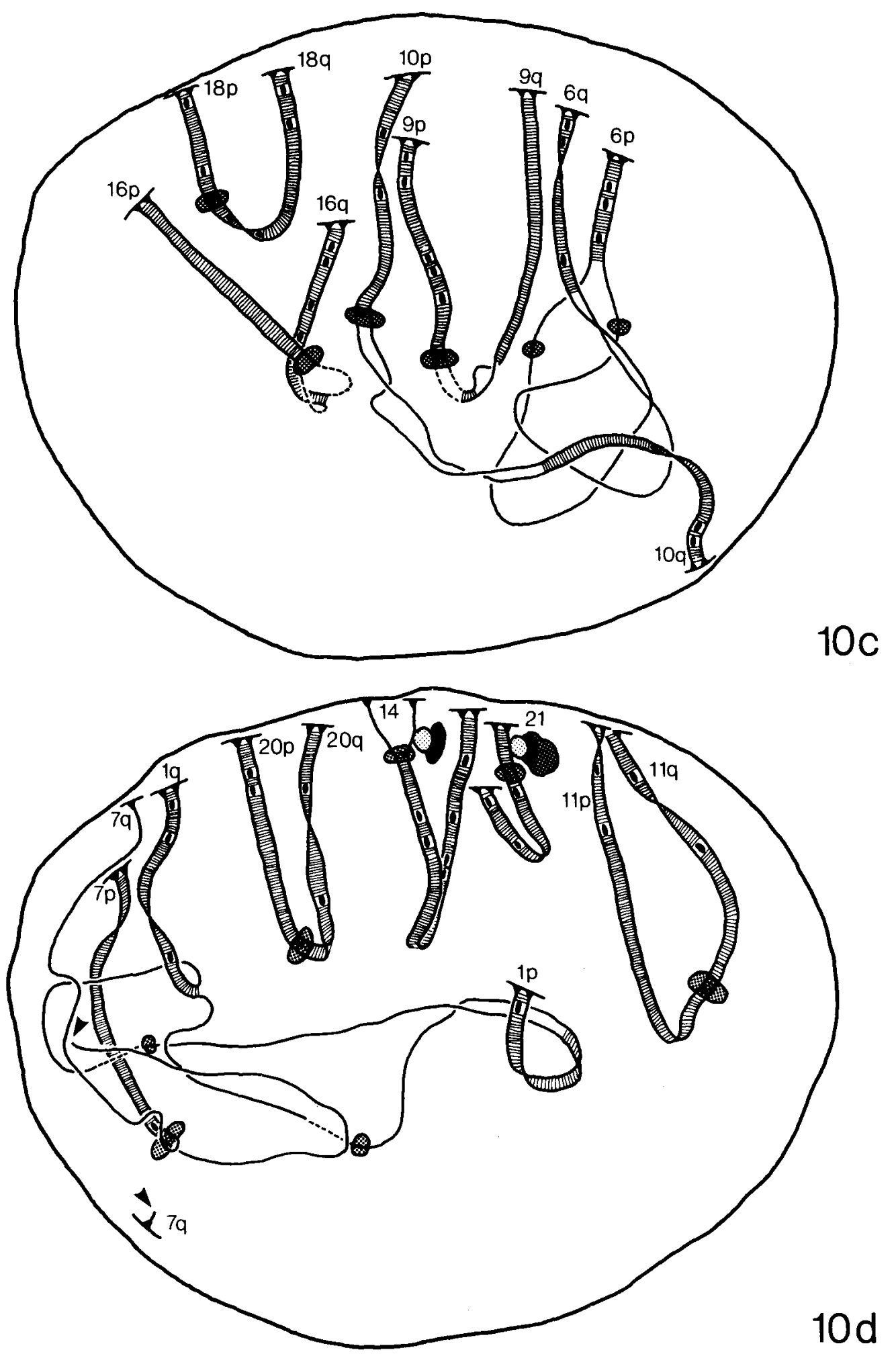

centromere. The short arms of chromosomes 13,14 and 15 are not yet paired. The homologous regions of the $X$ and $Y$ chromosomes are also unpaired (Figure $10 \mathrm{~b}$ ). Note that the $\mathrm{X}$ chromosome forms a loop around bivalents 3 and 15 and that the $X Y$ body characteristic of the pachytene stage has not yet formed. Figure 10b shows an interlocking of chromosome 3 between the unpaired middle segments of bivalent 4 (arrow). A subterminal break of the lateral component of chromosome 7 is shown in Figure 10d. The broken lateral component of chromosome 7 is located in a loop of an unpaired region of the short arm of chromosome 1 (arrow). The other arrow denotes the telomeric fragment of chromosome 7 , anchored at the nuclear envelope. The two ends of chromosome 7 are separated by a distance of $4.0 \mu \mathrm{m}$. (See also Figure 22.) 


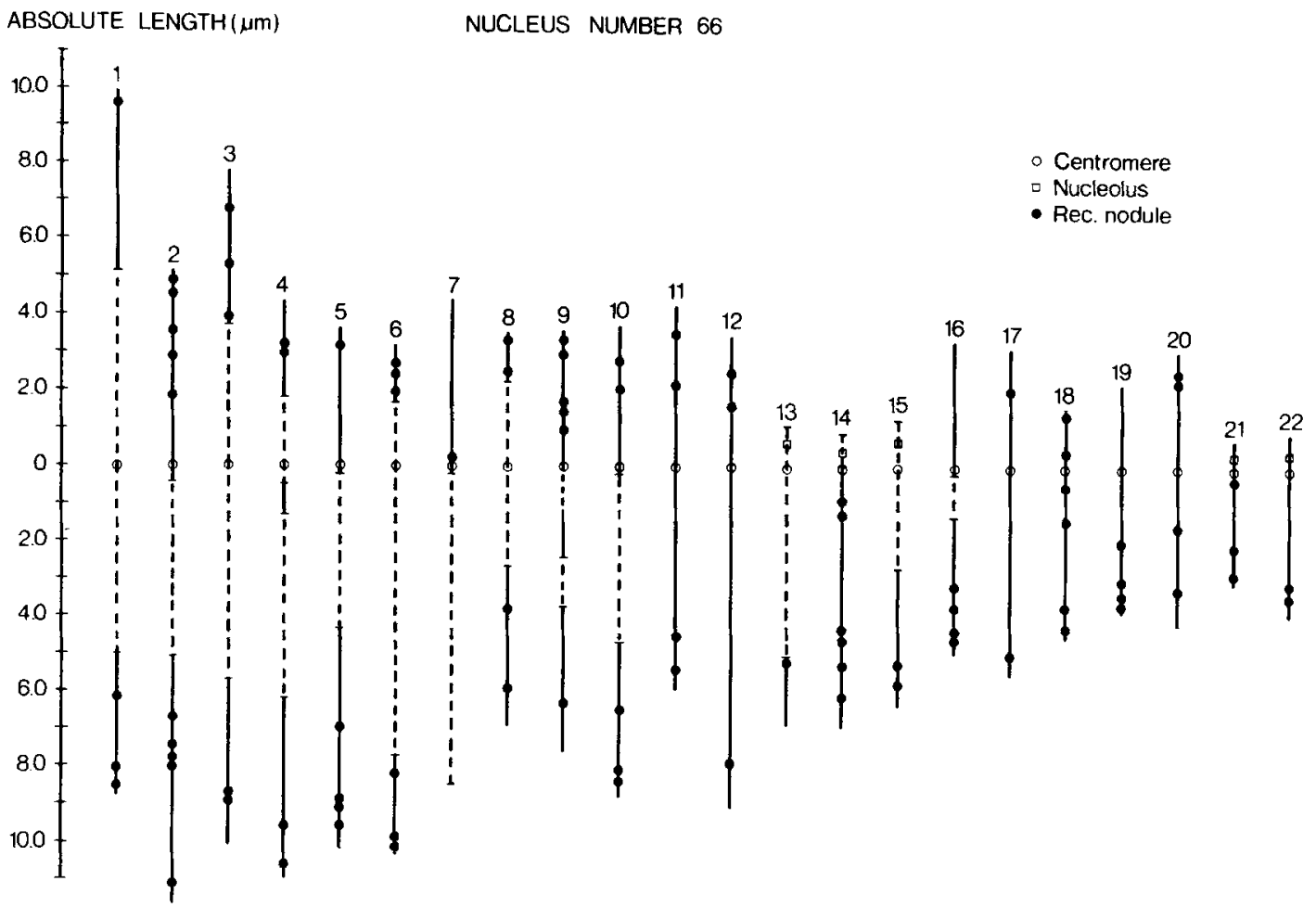

Figure 11. Idiogram of the autosome complement of a late zygotene nucleus (nucleus number 66, Figures 10a-d). The paired regions are shown as continuous lines, and unpaired regions as broken lines. Note that the recombination nodules frequently occur in small clusters.

breakage of bivalent 5 may result from the resolution of a second interlocking between chromosomes 5 and 14 . In the two remaining cases, the free ends of broken chromosomes and bivalents are all located in the vicinity of paired regions of other bivalents. Case 5: As illustrated in Figure 19, the free end of bivalent 22 is close to the synaptonemal complex of the fully paired bivalent 3 , suggesting that bivalent 22 has previously been interlocked between the homologues of bivalent 3. Case 6: In Figure 20 the broken ends of one chromosome 14 are in the proximity of the pairing fork of bivalent 12 . The two broken ends appear to be nonhomologously paired by a short region of a synaptonemal complex-like structure, which contains a dense node resembling a recombination nodule.

The telomeres of interlocked chromosomes and bivalents as well as of the chromosome and bivalent fragments were with one exception attached to the inner membrane of the nuclear envelope. In only one case were the ends of apparently intact chromosomes free in the nucleoplasm (Figure 21). It was, however, not possible to determine unequivocally whether the three free ends of the two homologues of bivalent 22 possessed intact telomeres or whether subterminal breaks had occurred. In the latter case, the telomeric fragments may have been lost as such fragments were not found attached to the nuclear envelope as was the case with the subterminal break in chromosome 7 in Figure 10d.

The number of interlockings and breaks recorded in the 10 zygotene nuclei can be regarded as a minimum number. In some cases two nonhomologous chromosomes or bivalents were located in the vicinity of each other, but the unpaired lateral components or the synaptonemal complexes were diffuse or not recognizable, and it was then not possible to reconstruct the chromosomes in these regions (Figure 14). Some of these configurations may represent additional situations of resolution of 

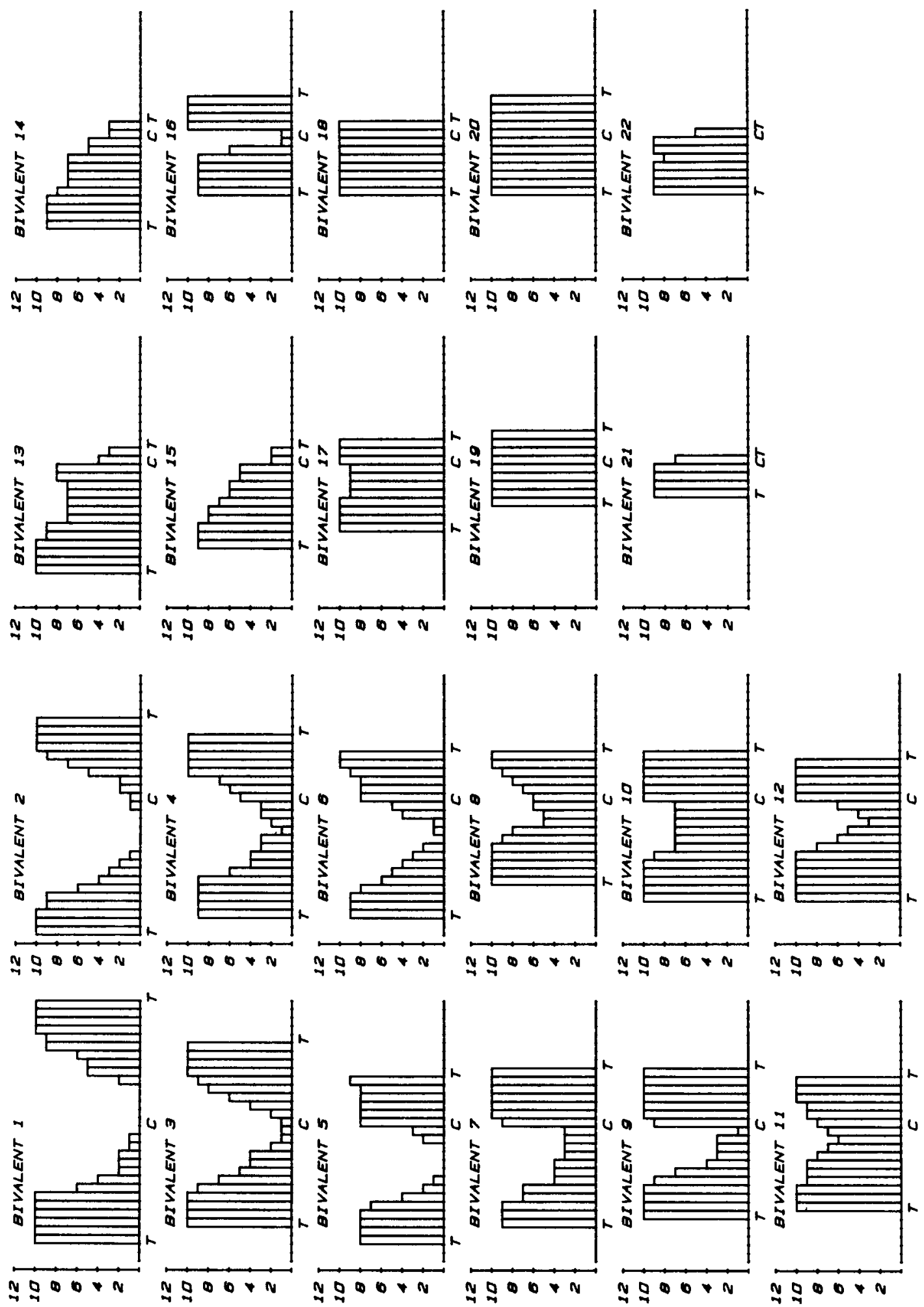

Figure 12. Histograms illustrating the extent of chromosome pairing in 10 late zygotene nuclei. The mean lengths of the partially paired bivalents are shown on the $\mathrm{X}$-axis with $\mathrm{T}$ and $\mathrm{C}$ denoting the position of the telomeres and the centromere respectively. The short arms of the bivalents are shown to the right and the long arms to the left, each arm being divided into $0.67 \mu \mathrm{m}$ intervals. The scale on the $Y$-axis shows the number of nuclei in which a particular chromosome segment is paired. Taking the long arm of bivalent 1 as an example, it can be seen that the six segments of bivalent 1 are paired in all 10 nuclei. In segment number 7, pairing closest to the telomere of the long arm was only observed in 6 nuclei. 
Table III

Chromosome aberrations and interlockings in 10 zygotene nuclei.

\begin{tabular}{|c|c|c|}
\hline $\begin{array}{l}\text { Nucleus } \\
\text { number }\end{array}$ & $\begin{array}{l}\text { Chromosomes } \\
\text { involved }\end{array}$ & Type of aberration \\
\hline \multirow[t]{2}{*}{66} & 1,7 & $\begin{array}{l}\text { One homologue of bivalent } 7 \text { broken and trapped between the two unpaired } \\
\text { lateral components of bivalent } 1 \text {. }\end{array}$ \\
\hline & 3,4 & The homologues of bivalents 3 and 4 interlocked \\
\hline 67 & 1,6 & The homologues of bivalents 1 and 6 interlocked \\
\hline 68 & $\begin{array}{c}2,10 \\
14\end{array}$ & $\begin{array}{l}\text { Bivalent } 10 \text { interlocked between the homologues of bivalent } 2 \text {. } \\
\text { One homologue of bivalent } 14 \text { broken and possibly nonhomologously paired } \\
\text { with itself. }\end{array}$ \\
\hline 69 & & No aberrations \\
\hline 70 & 5,14 & Bivalent 5 broken. Bivalent 14 interlocked by the homologues of bivalent 5 . \\
\hline 71 & & No aberrations \\
\hline 72 & 5,13 & $\begin{array}{l}\text { Resolving interlocking between one homologue of bivalent } 13 \text { and the unpaired } \\
\text { homologues of bivalent } 5 \text {. The interlocked homologue of bivalent } 13 \text { broken. }\end{array}$ \\
\hline 73 & $\begin{array}{c}21 \\
2,16 \\
2,4\end{array}$ & $\begin{array}{l}\text { Three of the four telomeres of bivalent } 21 \text { free. } \\
\text { Bivalent } 2 \text { interlocked by the homologues of bivalent } 16 \text {. } \\
\text { The homologues of bivalents } 2 \text { and } 4 \text { interlocked. }\end{array}$ \\
\hline 74 & $\mathrm{x}$ & $\mathrm{X}$ broken. \\
\hline 75 & 22 & Bivalent 22 broken. \\
\hline
\end{tabular}

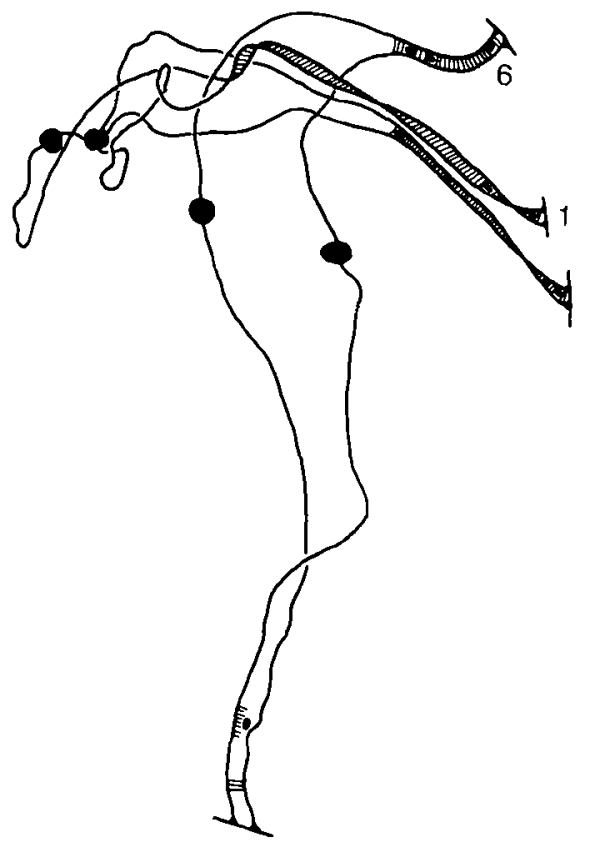

interlockings by breakage of lateral components of chromosomes and synaptonemal complexes of bivalents.

\subsection{Early pachytene}

\subsubsection{General observations}

The results presented below are based on complete reconstructions of 14 nuclei from three normal males and 7 nuclei (cf. section 2.1.) from a translocation heterozygote (22). Differences in ultrastructure of early pachytene nuclei from the three normal males and the

Figure 13. Reconstruction of an interlocking involving partially paired chromosomes 1 and 6 in a late zygotene nucleus (nucleus number 67 , Table III and Figure 7). The drawing is turned $90^{\circ}$ when compared to the reconstruction of the whole nucleus in Figure 7. 


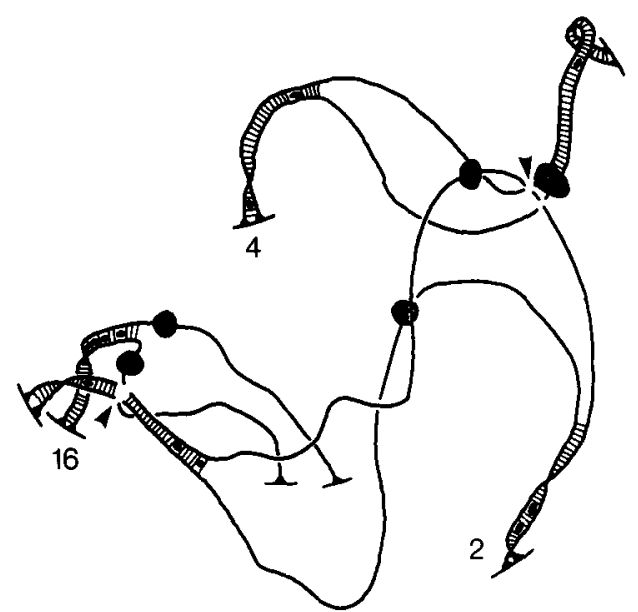

Figure 14. Reconstruction of a possible double interlocking involving bivalent 2 with bivalents 4 and 16 in a late zygotene nucleus (nucleus number 73 , Table III). Bivalent 2 appears to be interlocked between the lateral components of bivalent 16 . In addition, one of the lateral components of bivalent 2 is probably interlocked between the lateral components of bivalent 4 . The discontinuities of synaptonemal complexes and lateral components denoted by arrows may indicate the first step in the resolution of the interlocking by breakage of chromosomes and/or bivalents.

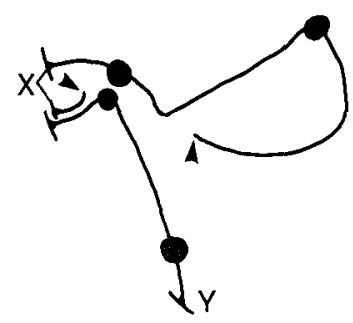

Figure 16. Reconstruction of the lateral component of the $\mathrm{X}$ chromosome in a late zygotene nucleus (nucleus number 74 , Table III) showing a subterminal break. The ends denoted by arrows are separated by a distance of $1.7 \mu \mathrm{m}$. The $X$ and $Y$ chromosomes are completely unpaired. The knob on the $\mathrm{Y}$ chromosome closest to the $\mathrm{X}$ chromosome marks the position of the centromere.

Figure 18. Reconstruction of the broken parts of the synaptonemal complex in bivalent 5 and one broken lateral component of chromosome 14 in a late zygotene nucleus (nucleus number 70 , Table III). The broken ends of bivalent 5 are separated by a distance of $0.5 \mu \mathrm{m}$ and the broken ends of chromosome 14 by a distance of $3.1 \mu \mathrm{m}$. The broken end of chromosome 14 is located between two unpaired segments of the homologues of bivalent 5 .

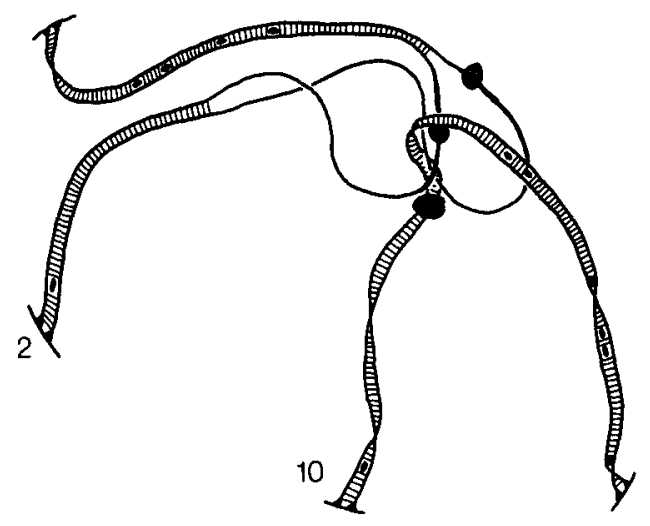

Figure 15. Reconstruction of an interlocking of bivalent 10 between the lateral components of bivalent 2 in a late zygotene nucleus (nucleus number 68, Table III).

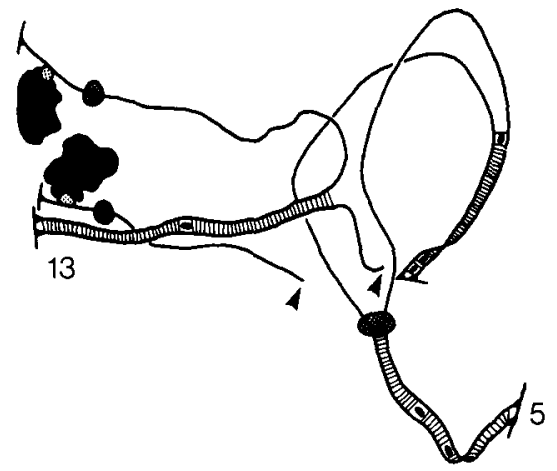

Figure 17. Reconstruction of chromosomes 5 and 13 (nucleus number 72, Table III). One lateral component of bivalent 13 is broken (arrows) and one of the broken ends is located between the unpaired lateral components of bivalent 5 .

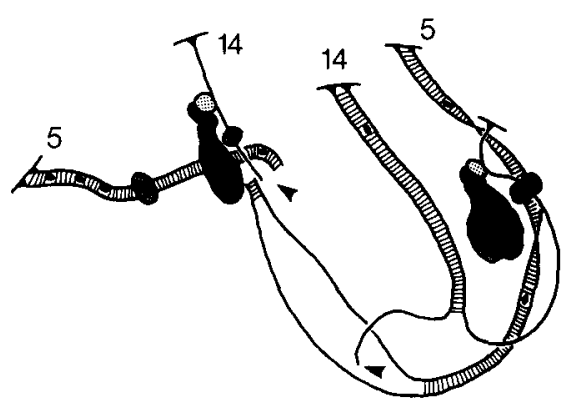




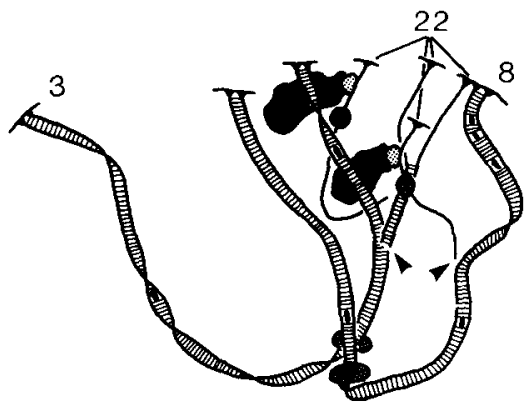

Figure 19. Reconstruction of the broken parts of the synaptonemal complex in bivalent 22 in a late zygotene nucleus (nucleus number 75, Table III). A segment of the long arm of bivalent 22 is paired interstitially and both homologues are broken (arrows). The bivalent has possibly been interlocked with bivalent number 3 . In addition, one of the chromosomes 22 may also have been interlocked with bivalent 8 . The ends denoted by arrows are lying very close to the synaptonemal complexes of bivalents 3 and 8 .

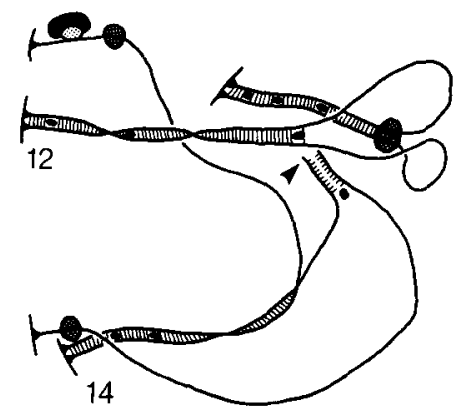

Figure 20. Reconstruction of chromosomes 14 in a late zygotene nucleus (nucleus number 68 , Table III). One lateral component of bivalent 14 is broken (arrow) and possibly nonhomologously paired with itself. The break may be caused by a resolution of an interlocking between bivalents 12 and 14 .

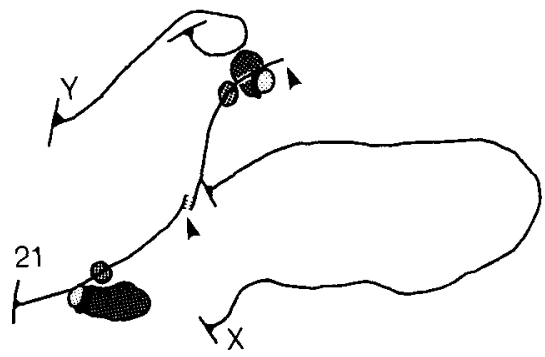

Figure 21. Reconstruction of chromosomes 21 in a late zygotene nucleus (nucleus number 73, Table III). The telomeres of both long arms and one of the short arms are free in the nucleoplasm (arrows). The terminal segments of the long arms are possibly paired. The $\mathrm{X}$ and $\mathrm{Y}$ chromosomes are located far apart.
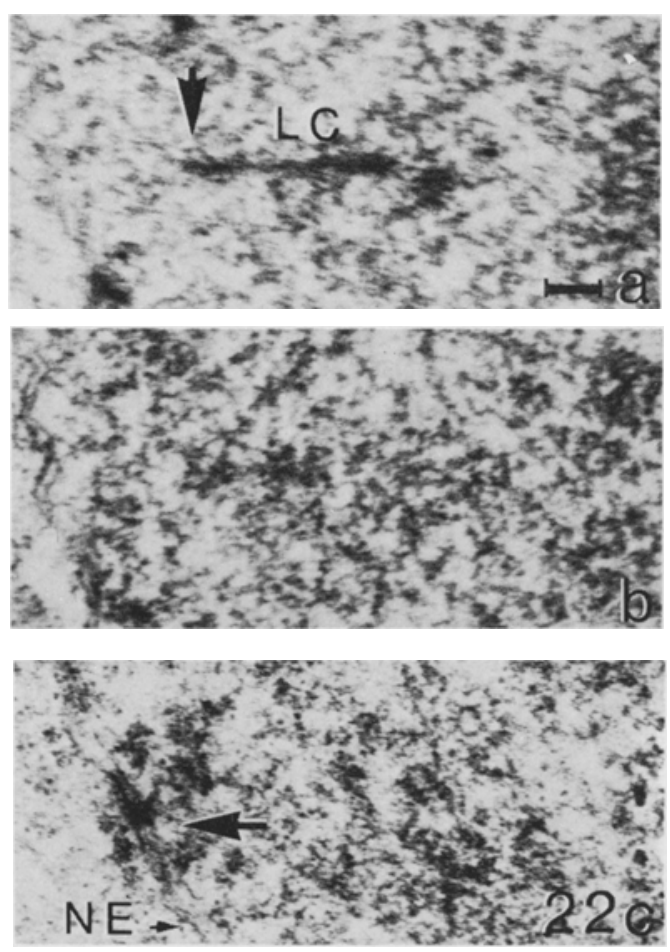

Figure 22. Micrographs showing a subterminal break of the lateral component of chromosome 7 in a late zygotene nucleus (nucleus number 66, Table III, and Figure 10d). Figures 22a and $b$ are micrographs of two consecutive sections showing the free end of the lateral component of chromosome 7 (arrow). Figure $22 \mathrm{c}$ shows the telomere region of chromosome 7 , attached to the nuclear envelope at a distance of $4.0 \mu \mathrm{m}$ from the end depicted in Figures 22a and $b$. The lateral component of the fragment extends $0.1 \mu \mathrm{m}$ into the nucleoplasm. LC, lateral component; NE, nuclear envelope.

$($ Bar $=0.2 \mu \mathrm{m})$

translocation heterozygote were not found - excepting the translocation chromosomes - and a comparison of the mean lengths and centromere indices of the bivalents from the two groups of nuclei revealed no significant differences (two-sample t-test, 5\% probability level). The total length of the synaptonemal complex complement amounted to $217 \pm 21 \mu \mathrm{m}$ in the translocation heterozygote as compared to 203 $\pm 23 \mu \mathrm{m}$ in the normal males, the difference being nonsignificant. Finally, a comparison between the number of recombination nodules per bivalent in the two groups did not reveal 
significant differences at a $2 \%$ probability level except for bivalent 5 , one of the translocation chromosomes. The mean number of recombination nodules in bivalent 5 in normal spermatocytes was $3.4 \pm 2.1$ whereas $5.6 \pm 1.9$ nodules were present in the translocation heterozygote. The distribution of recombination nodules along the individual bivalents appeared to be similar in the two groups although the number of nuclei was insufficient for a reliable statistical analysis. The total number of recombination nodules per nucleus in early pachytene was $74 \pm 11$ in the translocation heterozygote and $75 \pm 24$ in normal spermatocytes. This difference is not significant. It thus appears that the presence of the translocation between chromosomes 5 and 22 does not significantly change the bivalent lengths and centromere indices, nor does it alter the number and distribution of recombination nodules in the remainder of the genome. The ultrastructure of an early pachytene nucleus is shown in Figures 23a and $b$ and a reconstruction of the bivalents of another nucleus is shown in Figure 24. An idiogram of the autosomal bivalents of this nucleus is presented in Figure 25.

The early pachytene stage, or more correctly the zygotene-pachytene transition, is characterized by an almost complete pairing of all bivalents with a synaptonemal complex. The region of centromeric heterochromatin is characterized by presence of chromatin inside the synaptonemal complex as in late zygotene bivalents. Only the secondary constrictions of bivalents 1 and 9 are frequently without a complex at this stage (Figure 26), while that of bivalent 16 has a completed complex in all 21 nuclei. The secondary constrictions of bivalents 9 and 1 lacked complete complexes in 12 nuclei and 9 nuclei, respectively.

The telomeres of the bivalents are with one exception (Figure 29) anchored at the inner membrane of the nuclear envelope and the attachment sites are generally exhibiting a polarized distribution on the nuclear envelope. A clear chromosome bouquet was present in 14 nuclei, in 4 nuclei the bouquet was less pronounced, while in three nuclei the attachment sites appeared to be evenly distributed (compare Figures 24 and 29).
Each of the acrocentric bivalents were at this stage associated with a nucleolus. In addition, 0-3 nucleoli were present in the nucleoplasm and were occasionally located in the vicinity of the secondary constrictions of bivalents 1,9 , and 16. As illustrated in Figures 23, 24, 27 and 28 the chromatin of the XY bivalent has condensed giving rise to a typical $X Y$ body, while the modification of the lateral components of the sex chromosomes characteristic of mid-late pachytene is not yet evident. Recombination nodules are prevalent in the central region of the autosomal synaptonemal complexes (Figure 39 and section 3.7.) as well as in the paired region of $X Y$ bivalents.

\subsubsection{The early pachytene karyotype}

The criteria used in the classification of the autosomal bivalents are the same as those used for late zygotene in section 3.3.2. The mean values for absolute and relative lengths and centromere indices are given in Table IV.

\subsubsection{Total length of autosomes}

The total length of the autosomal synaptonemal complexes from each of the 21 nuclei are given in Table $V$. The nuclei numbered 39-41 and 47-50 are from the translocation heterozygote and the remaining nuclei are from the three normal males. The total length varies between $165.1 \mu \mathrm{m}$ and $245.8 \mu \mathrm{m}$ with a mean value of $207 \pm 23 \mu \mathrm{m}$.

\subsubsection{The XY bivalent}

In all early pachytene nuclei, the four telomeres of the $\mathrm{X}$ and $\mathrm{Y}$ chromosomes are close together on the nuclear envelope and a piece of synaptonemal complex is present between the homologous regions of the $\mathrm{X}$ and $\mathrm{Y}$ chromosomes (Figures 23, 24, 27 and 28). The length of the synaptonemal complex varies from 0.4-1.9 $\mu \mathrm{m}$ (Table VIII) with a mean value of $0.9 \pm 0.4$ $\mu \mathrm{m}$. In one of the nuclei the central region of the complex had not yet attained its normal ultrastructural characteristics, the two lateral components being separated by a distance of $150 \mathrm{~nm}$ (Figure 29). The synaptonemal complex 


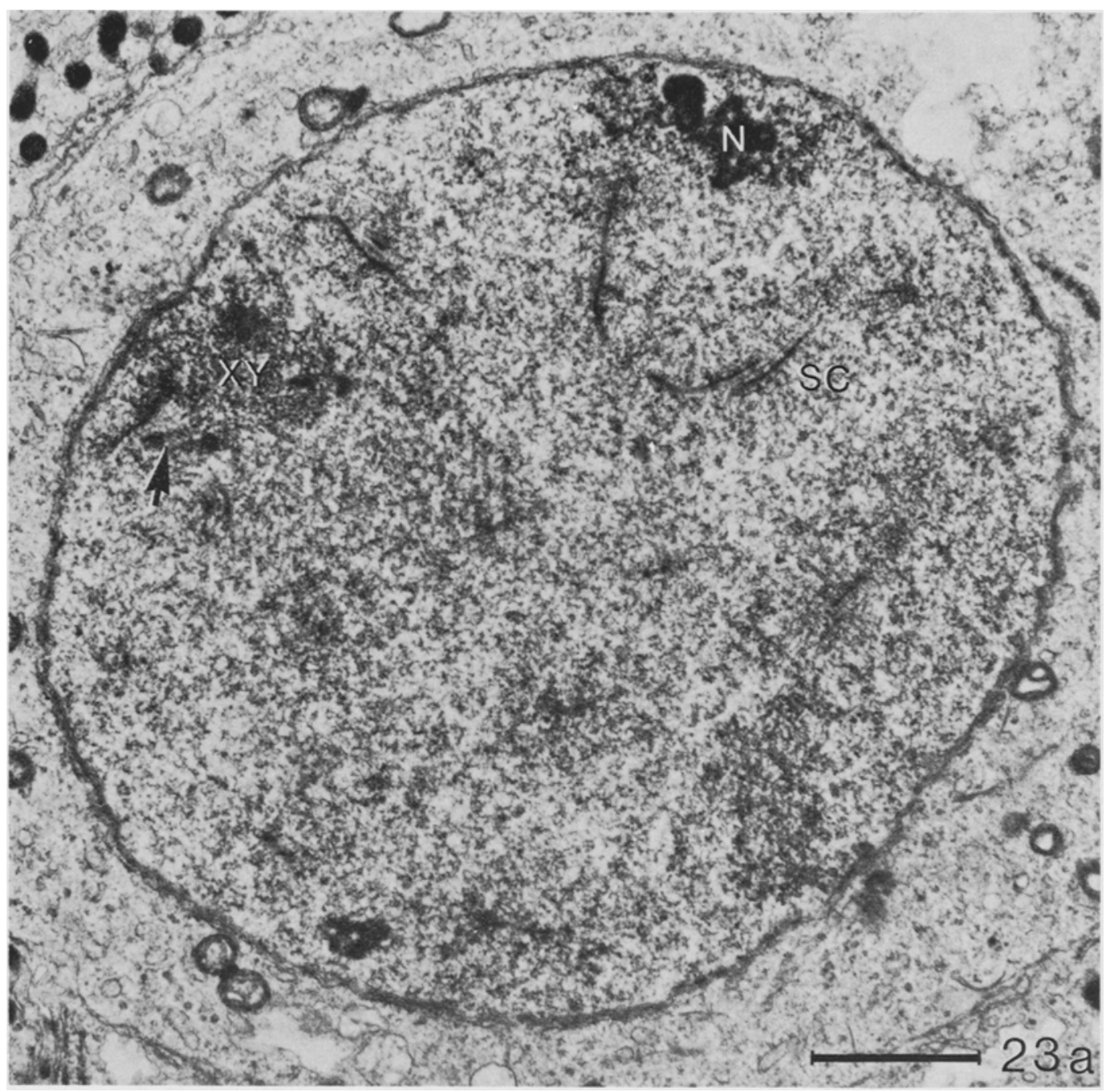

Figure 23. Survey micrographs of two consecutive sections of an early pachytene nucleus (nucleus number 54). Long stretches of synaptonemal complex (SC) are present whereas unpaired lateral components of autosomes cannot be recognized. The $\mathrm{X}$ and $\mathrm{Y}$ chromosomes are paired over a short distance with a synaptonemal complex (arrow) while the unpaired segments of the sex chromosomes have condensed into a discrete $X Y$ body $(X Y)$. The nucleolus $(\mathrm{N})$ is connected to the short arm of an acrocentric bivalent through a region of high electron density and a filamentous body $(\mathrm{FB})$.

$(\mathrm{Bar}=2 \mu \mathrm{m})$

was in 16 of the 22 cases associated with 1-2 recombination nodules, while in the remainder of the nuclei nodules were not identified in the paired region of the $\mathrm{X}$ and $\mathrm{Y}$ chromosomes.

The unpaired segments of the $\mathrm{X}$ and $\mathrm{Y}$ chromosomes possess continuous lateral components (Figure 28) about $60 \mathrm{~nm}$ in diameter which in cross and longitudinal sections oc- casionally appear double. The lateral component of the $\mathrm{Y}$ chromosome is associated with a heterochromatic knob proximal to the paired region, the knob marking the extreme limit of synaptonemal complex formation observed. It is assumed without direct evidence that this knob corresponds to the centromeric heterochromatin of the $\mathrm{Y}$ chromosomie. The centro- 


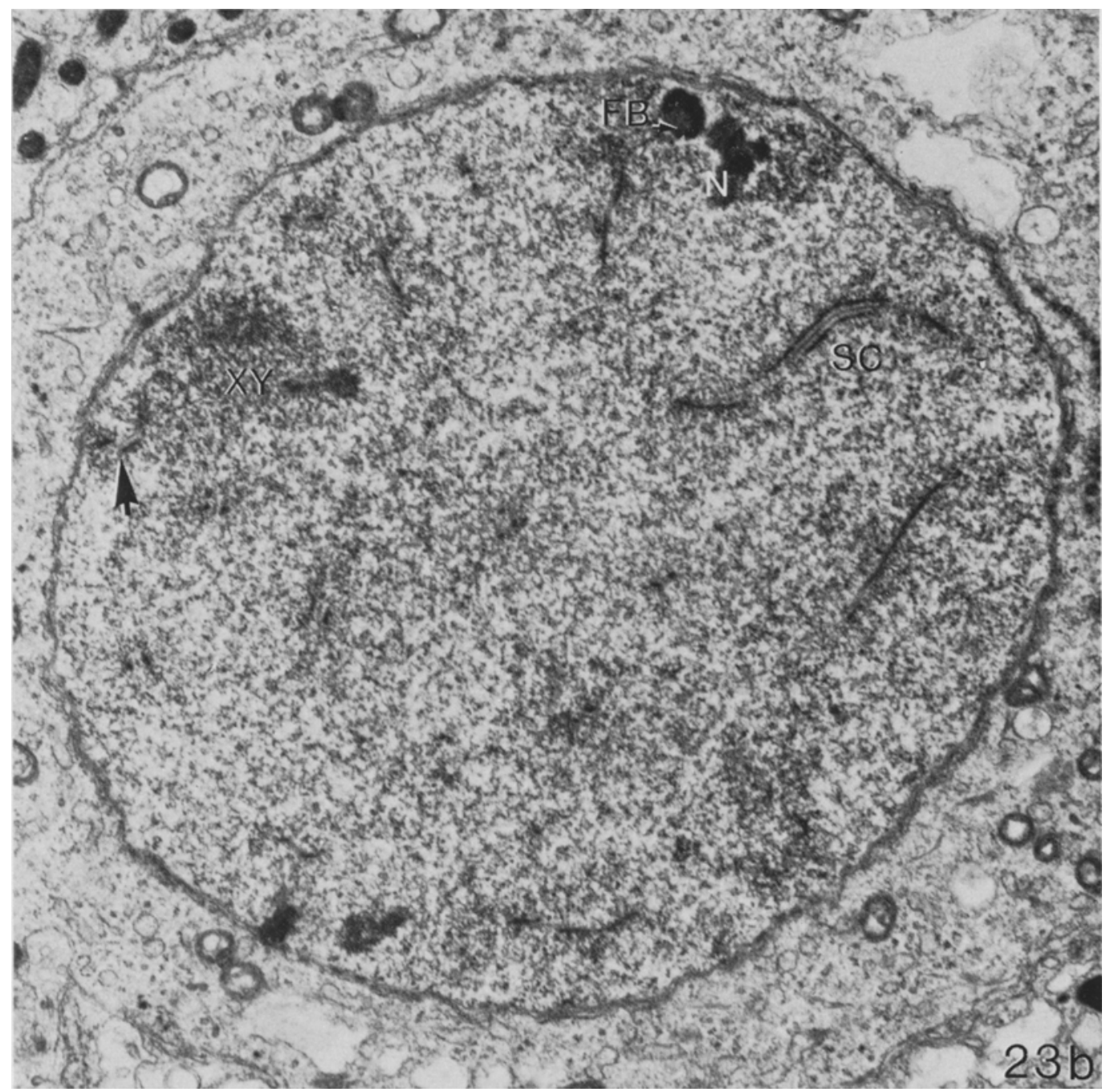

mere index calculated on this assumption is 30 as compared to a centromere index of 23-27 reported at the Paris Conference (39) on the basis of light microscopical identification of the centromere region of mitotic chromosomes. The remaining part of the $\mathrm{Y}$ chromosome, the long arm, was with one exception associated with a large nucleolus-like structure, as illustrated in Figure 28, being in contact with the lateral component approximately in the middle of the long arm.

The ultrastructure of the $\mathrm{X}$ chromosome is more variable and up to six heterochromatic knobs may be present on the lateral component. The morphological similarity of the knobs and the centromeric heterochromatin makes an identification of the position of the centromere ambiguous.

In one nucleus (number 63, Figure 29) the chromatin has not yet attained the ultrastructure seen in the other early pachytene nuclei and the $\mathrm{Y}$ chromosome is not associated with nucleolus-like material. As in late zygotene, a heterochromatic knob is present in the middle of the long arm. 


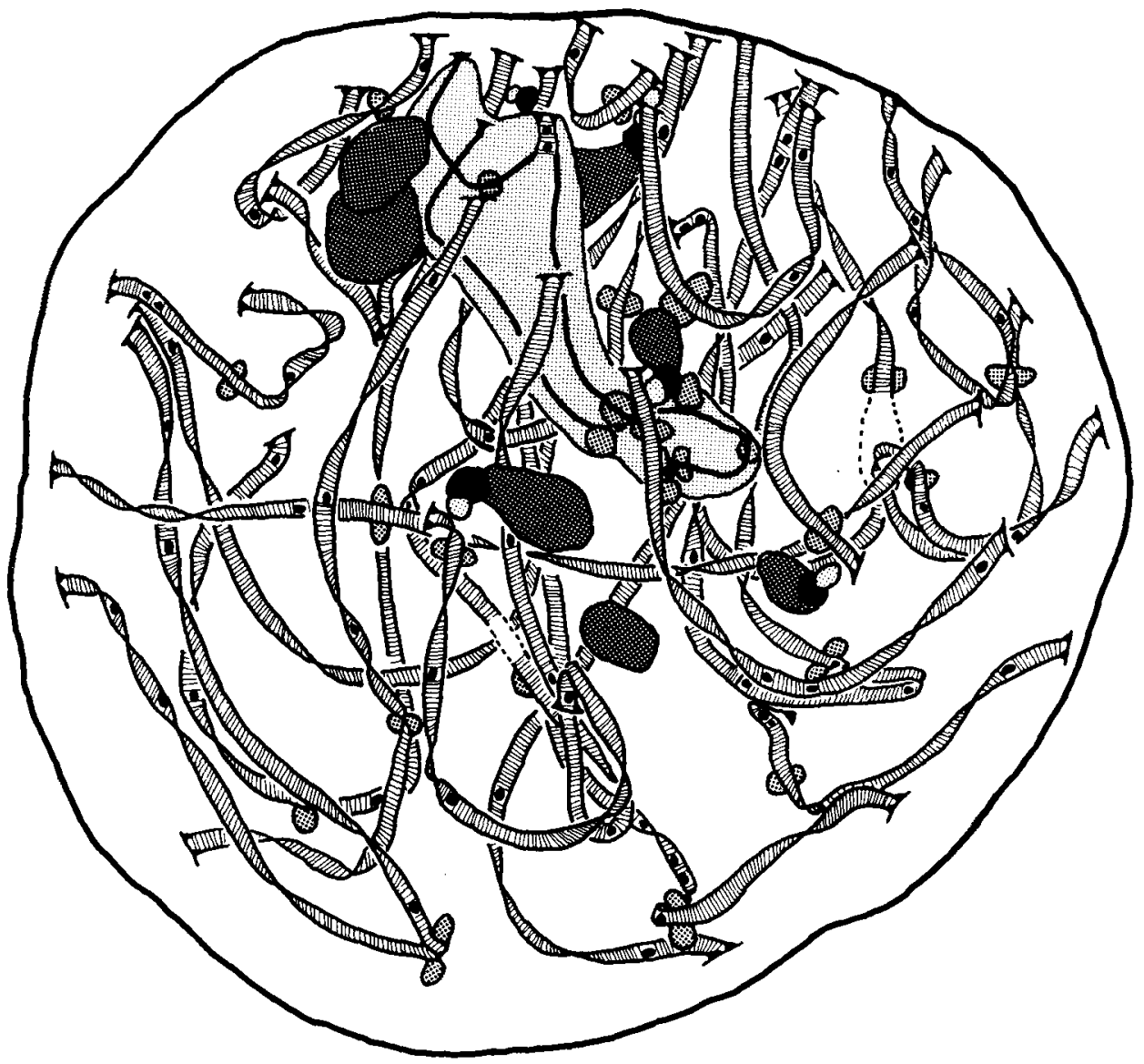

Figure 24. A complete reconstruction of an early pachytene nucleus (nucleus number 59). With the exceptions of the secondary constrictions of bivalents 1 and 9 the autosomal bivalents are all paired with continuous synaptonemal complexes from telomere to telomere. The broken lines in the reconstructions indicate the discontinuous lateral components of bivalents 1 and 9 . Note the bouquet configuration and the large number of recombination nodules (99, Table $\mathrm{V}$ ). The $\mathrm{X}$ and $\mathrm{Y}$ chromosomes are paired with a synaptonemal complex in a short region near the telomere. The paired segment contains two recombination nodules attached to the central region of the complex. The chromatin of the XY bivalent is shown with a light hatching, centromeric heterochromatin with an intermediate hatching and nucleoli with a dark hatching (see also Figures 25 and 28).

\subsubsection{Interlockings and breaks}

Two cases of bivalent interlocking were revealed in one of the 21 early pachytene nuclei (number 63, Figures 29-33). As illustrated in Figure 33, bivalent 4 is interlocked between the homologues of bivalent 1 , the long unpaired region of the latter exhibiting the morphological characteristics of unpaired regions of the $X Y$ bivalent of early pachytene, i.e., thickening of the lateral components and relatively condensed chromatin. These modifications of the lateral components and the chromatin were lacking in the second interlocking in this nucleus (Figures 30-32). In nucleus number 62 the homologues of bivalent 2 were unpaired in a long region in the middle of the bivalent. The lateral components and the chromatin exhibited the same ultrastructural modifications as those described for bivalent 1. Apparently the mechanism responsible for the change in ultrastructure of the unpaired regions of the $\mathrm{XY}$ bivalent may also act on unpaired regions of the autosomes at this stage of meiosis.

Three cases of chromosome breakage $(1$ in 


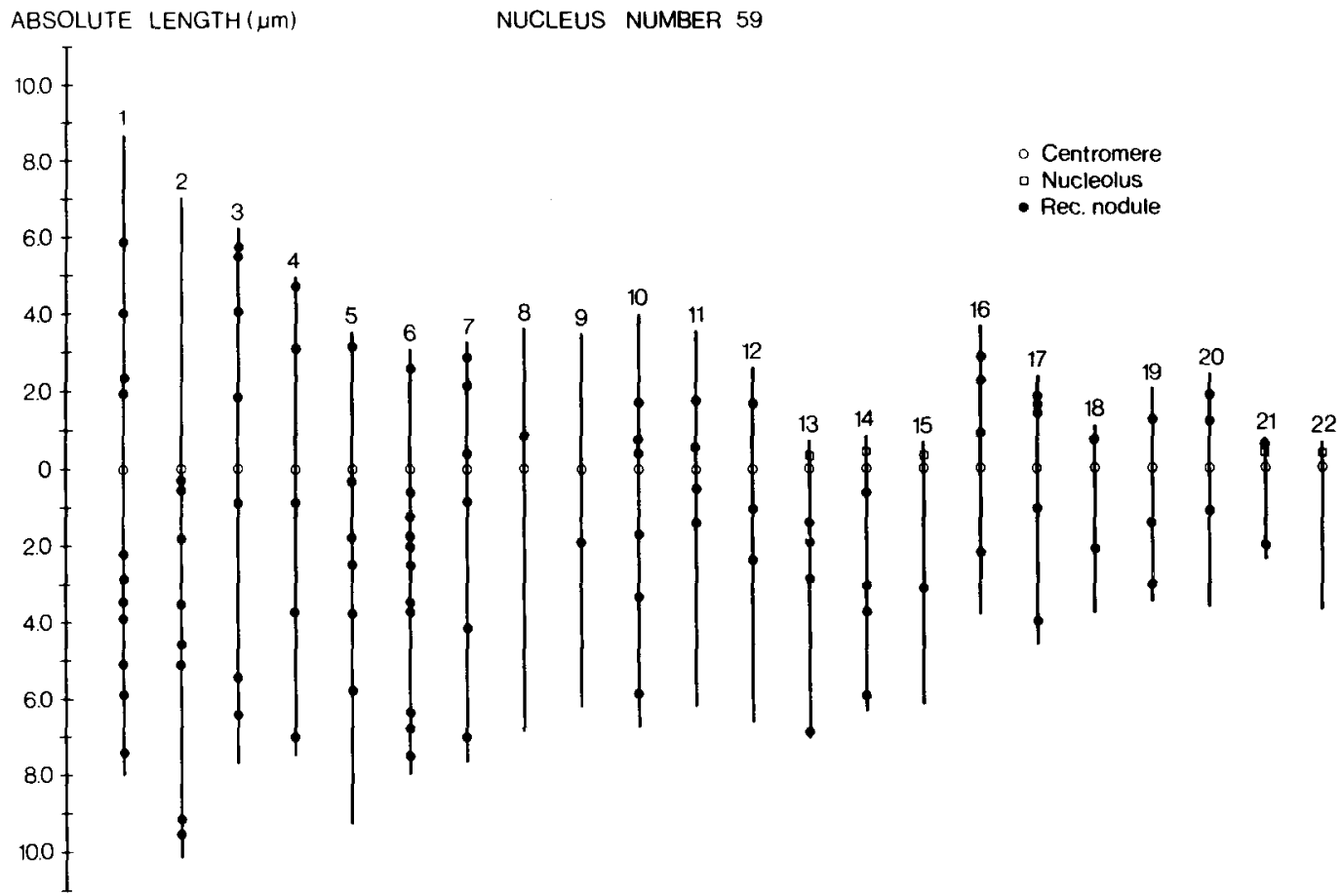

Figure 25. Idiogram of the autosomal complement of an early pachytene nucleus (nucleus number 59, Figure 24). Note the absence of recombination nodules in the short arms of bivalents 2 and 9 , and in the long arm of bivalent 8. Bivalent 22 is completely devoid of nodules. A recombination nodule is present in the short arm of bivalent 21 . The short unpaired regions of the secondary constrictions in bivalents 1 and 9 are not shown in the idiogram.

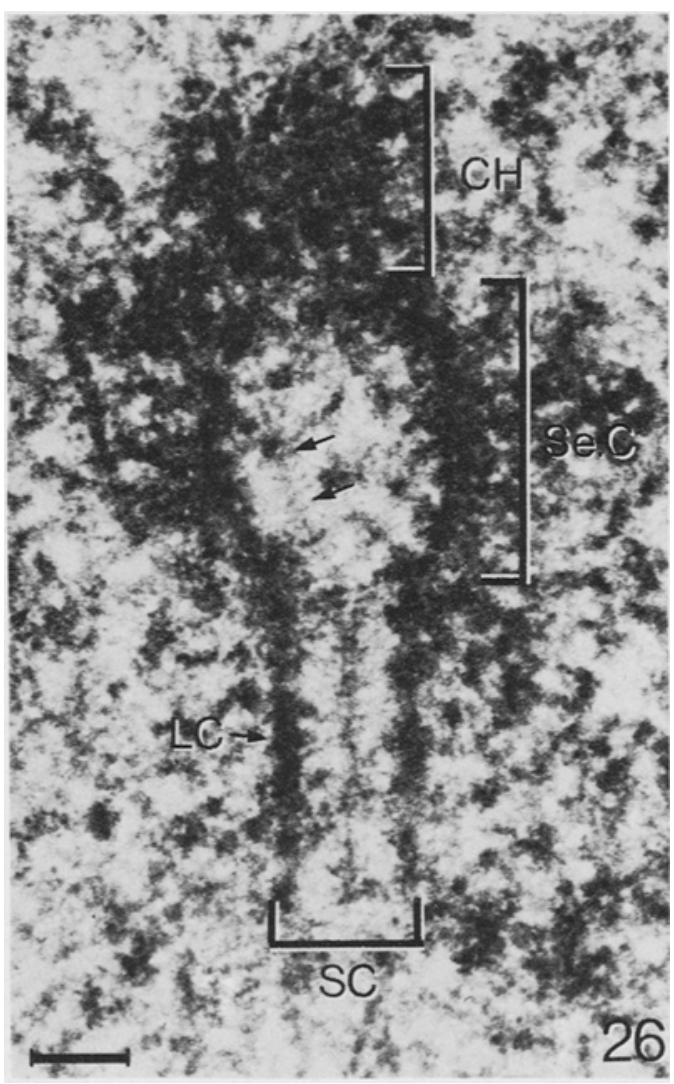

nucleus number 60 and 2 in nucleus number 58) have been identified in the 21 early pachytene nuclei. The break in the $Y$ chromosome (nucleus number 60 , Figure 34 ) is in the long arm between the centromeric heterochromatin and the point where the lateral component is associated with the nucleolus-like material. The combined length of the two fragments is $3.2 \mu \mathrm{m}$ compared to a mean length of $3.6 \pm 0.7 \mu \mathrm{m}$ for the $\mathrm{Y}$ chromosome (Table VIII). In nucleus number 58, two chromosome breaks were found, one in the long arm of one homologue of bivalent 1 and the other in the $\mathrm{Y}$ chromosome.

Figure 26. Electron micrograph showing the unpaired secondary constriction of bivalent 1 from an early pachytene nucleus. The two homologous chromosomes loop out and are unpaired in the region proximal to the centromeric heterochromatin $(\mathrm{CH})$. Note that a central region $(\mathrm{CR})$ is organized and associated with only one of the lateral components within the unpaired region (arrow).

Se. C., secondary constriction

LC, lateral component

$\mathrm{SC}$, synaptonemal complex

$(\mathrm{Bar}=0.1 \mu \mathrm{m})$ 
Table IV

Mean values of absolute length, relative length, centromere index, and number of recombination nodules for the 22 autosomal bivalents of 21 pachytene nuclei.

\begin{tabular}{|c|c|c|c|c|c|c|c|c|}
\hline $\begin{array}{l}\text { Bivalent } \\
\text { number }\end{array}$ & $\begin{array}{l}\text { Absolute } \\
\text { length }(\mu \mathrm{m})\end{array}$ & $\begin{array}{l}\text { Standard } \\
\text { deviation }\end{array}$ & $\begin{array}{l}\text { Relative } \\
\text { length (\%) }\end{array}$ & $\begin{array}{l}\text { Standard } \\
\text { deviation }\end{array}$ & $\begin{array}{c}\text { Centromere } \\
\text { index }\end{array}$ & $\begin{array}{l}\text { Standard } \\
\text { deviation }\end{array}$ & $\begin{array}{c}\text { Number of } \\
\text { nodules }\end{array}$ & $\begin{array}{l}\text { Standard } \\
\text { deviation }\end{array}$ \\
\hline 1 & 17.4 & 1.9 & 8.4 & 0.4 & 51 & 4 & 7.2 & 3.1 \\
\hline 2 & 15.8 & 2.6 & 7.9 & 0.3 & 40 & 3 & 6.0 & 2.6 \\
\hline 3 & 13.7 & 1.9 & 6.6 & 0.4 & 47 & 2 & 4.4 & 1.4 \\
\hline 4 & 12.3 & 1.5 & 5.9 & 0.4 & 36 & 5 & 3.4 & 1.8 \\
\hline 5 & 12.4 & 1.5 & 5.9 & 0.3 & 29 & 4 & 4.2 & 2.2 \\
\hline 6 & 11.3 & 1.3 & 5.4 & 0.3 & 37 & 5 & 4.6 & 2.5 \\
\hline 7 & 10.9 & 1.4 & 5.3 & 0.3 & 38 & 6 & 4.5 & 2.7 \\
\hline 8 & 9.9 & 1.2 & 4.8 & 0.2 & 35 & 4 & 3.2 & 1.5 \\
\hline 9 & 10.3 & 1.4 & 5.0 & 0.4 & 37 & 6 & 3.2 & 1.9 \\
\hline 10 & 10.3 & 1.2 & 4.9 & 0.2 & 36 & 4 & 3.6 & 1.7 \\
\hline 11 & 9.3 & 1.3 & 4.5 & 0.3 & 36 & 6 & 3.2 & 1.7 \\
\hline 12 & 9.9 & 1.3 & 4.8 & 0.3 & 28 & 3 & 3.4 & 2.0 \\
\hline 13 & 8.0 & 1.1 & 3.8 & 0.3 & 12 & 5 & 2.9 & 2.2 \\
\hline 14 & 7.4 & 0.9 & 3.6 & 0.2 & 13 & 3 & 2.2 & 1.4 \\
\hline 15 & 6.9 & 0.8 & 3.3 & 0.2 & 11 & 2 & 2.6 & 1.7 \\
\hline 16 & 7.5 & 1.0 & 3.6 & 0.3 & 44 & 4 & 2.6 & 1.4 \\
\hline 17 & 7.8 & 1.3 & 3.8 & 0.3 & 33 & 3 & 2.8 & 1.7 \\
\hline 18 & 5.5 & 0.8 & 2.6 & 0.2 & 27 & 5 & 2.2 & 1.3 \\
\hline 19 & 5.4 & 0.7 & 2.7 & 0.3 & 42 & 4 & 2.0 & 1.0 \\
\hline 20 & 7.0 & 0.8 & 3.4 & 0.3 & 43 & 4 & 2.5 & 1.4 \\
\hline 21 & 3.5 & 0.5 & 1.7 & 0.1 & 23 & 6 & 1.0 & 0.7 \\
\hline 22 & 4.5 & 0.8 & 2.1 & 0.2 & 21 & 7 & 1.6 & 1.1 \\
\hline
\end{tabular}

The break in the $\mathrm{Y}$ chromosome is proximal to the centromeric heterochromatin and a synaptonemal complex is absent between the $\mathrm{X}$ and $Y$ chromosomes, suggesting a loss of the homologous segment, i.e., the short arm of the $Y$ chromosome. The length of the remainder of the $\mathrm{Y}$ chromosome is $2.5 \mu \mathrm{m}$ and is identical to the mean length of the long arm. The broken arm of chromosome 1 was not anchored at the nuclear envelope and was $1.1 \mu \mathrm{m}$ shorter than its homologue. A fragment was not found elsewhere in the nucleus, suggesting that the breakage had occurred before or during the last premeiotic mitosis with subsequent loss of the fragment. This break in the long arm of chromosome 1 appears also to have created a genuine terminal deletion. The unpaired regions of the homologues of bivalent 1 were intermingled with the $X$ and $Y$ chromosomes and have attained the ultrastructural characteristics of the latter with respect to chromatin and diameter of the lateral components (Figure 35).

\subsection{Comparison of the total lengths of the autosomes}

The mean total lengths of the autosomal complements at late zygotene, early pachytene and mid-late pachytene are presented in Table VI. The lengths were $235 \pm 26,207 \pm 23$, and $231 \pm 16 \mu \mathrm{m}$.

A comparison using a two-sample t-test revealed a significant difference in length at a $1 \%$ probability level between late zygotene and early pachytene as well as between early pachytene and mid-late pachytene. 


\subsection{Comparison of bivalent lengths}

Idiograms illustrating the mean values for absolute lengths and centromere indices of the 22 autosomal bivalents at late zygotene, early pachytene and mid-late pachytene are presented in Figure 36. The centromere indices at the different stages appear very similar. Furthermore, all early pachytene bivalents are shorter than the ones at late zygotene and mid-late pachytene, the bivalents of the latter stages being very similar in length. The $12 \%$ decrease in length of the total autosomal complement noted at early pachytene is accordingly distributed proportionately among all bivalents.

A small decrease in length from late zygotene to early pachytene is noted for the $X$ and $Y$ chromosomes (Table VIII). Data are not avail-

\section{Table V}

Total complement length, total number of recombination nodules, and synaptonemal complex length per recombination nodule for 21 pachytene nuclei.

\begin{tabular}{|c|c|c|c|}
\hline $\begin{array}{l}\text { Nucleus } \\
\text { number }\end{array}$ & $\begin{array}{c}\text { Total } \\
\text { complement } \\
\text { length }(\mu \mathrm{m})\end{array}$ & $\begin{array}{c}\text { Total } \\
\text { number of } \\
\text { nodules }\end{array}$ & $\begin{array}{c}\text { SC length } \\
(\mu \mathrm{m}) \text { per } \\
\text { nodule }\end{array}$ \\
\hline 39 & 232.8 & 93 & 2.5 \\
\hline 40 & 212.2 & 71 & 3.0 \\
\hline 41 & 245.8 & 71 & 3.5 \\
\hline 47 & 179.6 & 80 & 2.2 \\
\hline 48 & 215.9 & 59 & 3.7 \\
\hline 49 & 209.6 & 68 & 3.1 \\
\hline 50 & 220.1 & 75 & 2.9 \\
\hline 43 & 201.7 & 65 & 3.1 \\
\hline 44 & 229.2 & 114 & 2.0 \\
\hline 54 & 205.6 & 40 & 5.1 \\
\hline 55 & 227.0 & 56 & 4.1 \\
\hline 56 & 206.9 & 58 & 3.6 \\
\hline 57 & 193.5 & 59 & 3.3 \\
\hline 58 & 183.6 & 107 & 1.7 \\
\hline 59 & 204.8 & 99 & 2.1 \\
\hline 60 & 211,6 & 69 & 3.1 \\
\hline 61 & 173.8 & 84 & 2.1 \\
\hline 62 & 168.8 & 57 & 3.0 \\
\hline 63 & 165.1 & 51 & 3.2 \\
\hline 64 & 228.7 & 79 & 2.9 \\
\hline 65 & 234.7 & 110 & 2.1 \\
\hline Mean & 207.2 & 75 & 3.0 \\
\hline $\begin{array}{l}\text { Standard } \\
\text { deviation }\end{array}$ & 22.8 & 20 & 0.8 \\
\hline
\end{tabular}

able for the mid-late pachytene nuclei as the lateral components of the sex chromosomes cannot be completely reconstructed at this stage (20). The length of the synaptonemal complex between the $\mathrm{X}$ and $\mathrm{Y}$ chromosomes at mid-late pachytene amounts to $0.4 \pm 0.4 \mu \mathrm{m}$ compared to $0.9 \pm 0.4 \mu \mathrm{m}$ at early pachytene. This difference is significant at $0.1 \%$ probability level and in agreement with the proposal by TREs (47). A temporal sequence of individual nuclei established on this criterium alone (47) is, however, unreliable as the length of this synaptonemal complex varies considerably in early pachytene (between 0.4 and $1.9 \mu \mathrm{m}$ ) as well as in mid-late pachytene (between 0.1 and $1.0 \mu \mathrm{m})$.

\subsection{Recombination nodules}

\subsubsection{Ultrastructure}

Recombination nodules are associated with the central region of the synaptonemal complex at all substages of zygotene as well as at early pachytene. At early zygotene structures resembling recombination nodules have been observed associated with unpaired lateral components or connected to the precursor material of the central region between grossly aligned lateral components (Figure 37a). Furthermore, recombination nodules are frequently present in or near the pairing fork (Figure 37b).

It is evident from Figures 37-39 that the dimensions and ultrastructure of recombination nodules are highly variable at all substages of zygotene as well as at early pachytene. In shape the nodules can range from spheres to rods and dumbbells. The shorter diameter varied from $30-60 \mathrm{~nm}$ and the longer from $30-150 \mathrm{~nm}$. The nodules were located either within the central region of the synaptonemal complex (Figure $38 \mathrm{f}$ ) or associated with the complex outside the plane defined by the two lateral components (Figures 37c, 38g and 39d). In both cases nodules appear to be connected to the lateral components by fibers (Figures 39a and d). The nodules seem to be composed of fibers with a density varying from that of the chromatin to a density similar to that of the lateral components. One gets the impression that nodules at early pachytene are frequently larger and more 

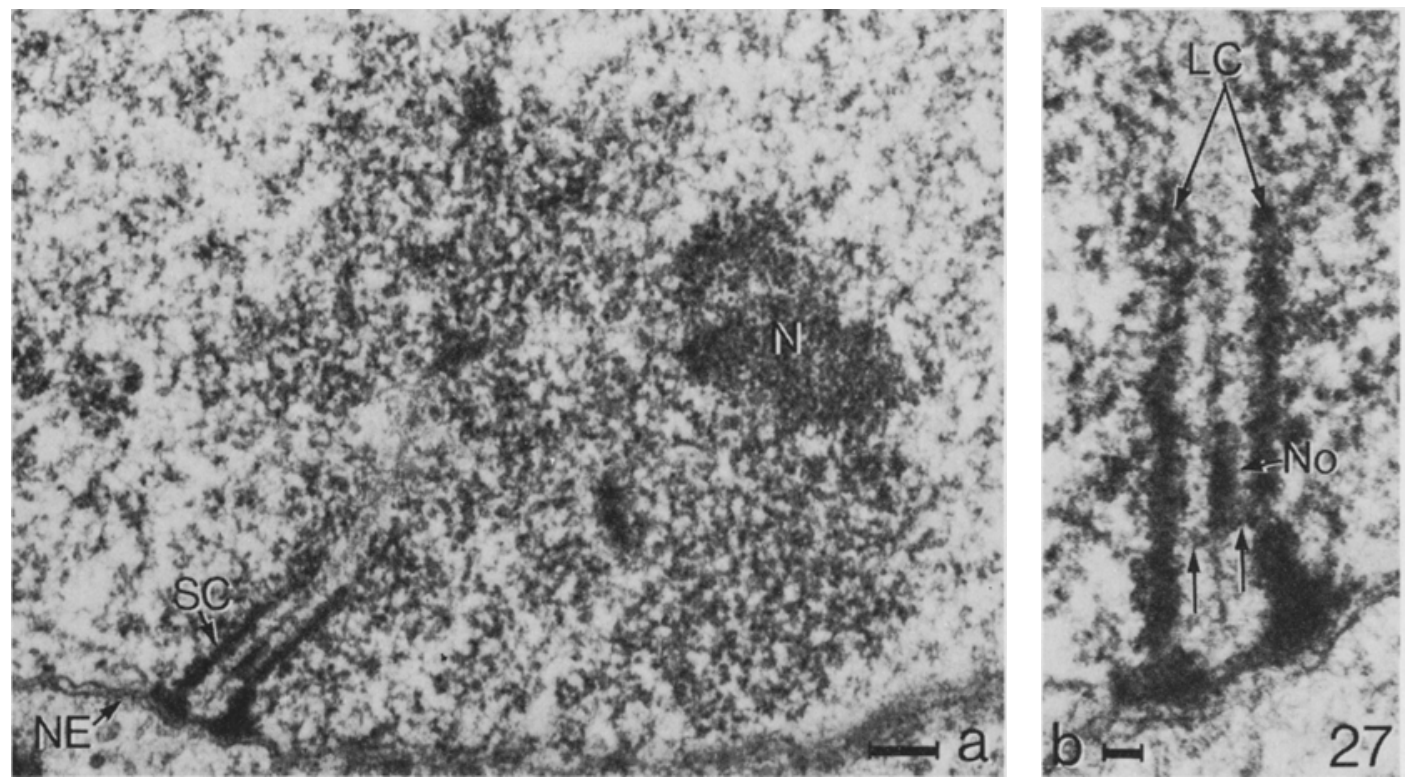

Figure 27. Electron micrographs of the $X Y$ bivalent at early pachytene. The $X$ and $Y$ chromosomes are paired over a short distance with a synaptonemal complex (SC) and attached to the inner membrane of the nuclear envelope (NE). Nucleolus-like (N) material is associated with the $\mathrm{Y}$ chromosome (see also Figure 28). Figure 27b is a higher magnification of the synaptonemal complex in Figure 27a. Note that the recombination nodule (No) is associated with the lateral components (LC) through fine fibers (arrows).

Figure 27a $(\mathrm{Bar}=0.2 \mu \mathrm{m})$

Figure $27 \mathrm{~b}(\mathrm{Bar}=0.05 \mu \mathrm{m})$

electron dense than the ones at earlier stages and that nodules more often are located outside the central region at early pachytene. It is unlikely that the differences in morphology and density of the recombination nodules can be accounted for by different planes of sectioning relative to the synaptonemal complex.

Figure 28. A reconstruction of the $X Y$ bivalent in an early pachytene nucleus (nucleus number 59, Figure 24). The telomeric regions of the $X$ and $Y$ chromosomes are paired with a synaptonemal complex over a distance of $0.4 \mu \mathrm{m}$. Two recombination nodules are present in the central region of the complex. A large block of nucleolus-like material (dark hatching) is associated with the $\mathrm{Y}$ chromosome. The small heterochromatic region marks the position of the centromere region of the $Y$ chromosome. Six regions of similar ultrastructure are associated with the lateral component of the $\mathrm{X}$ chromosome.

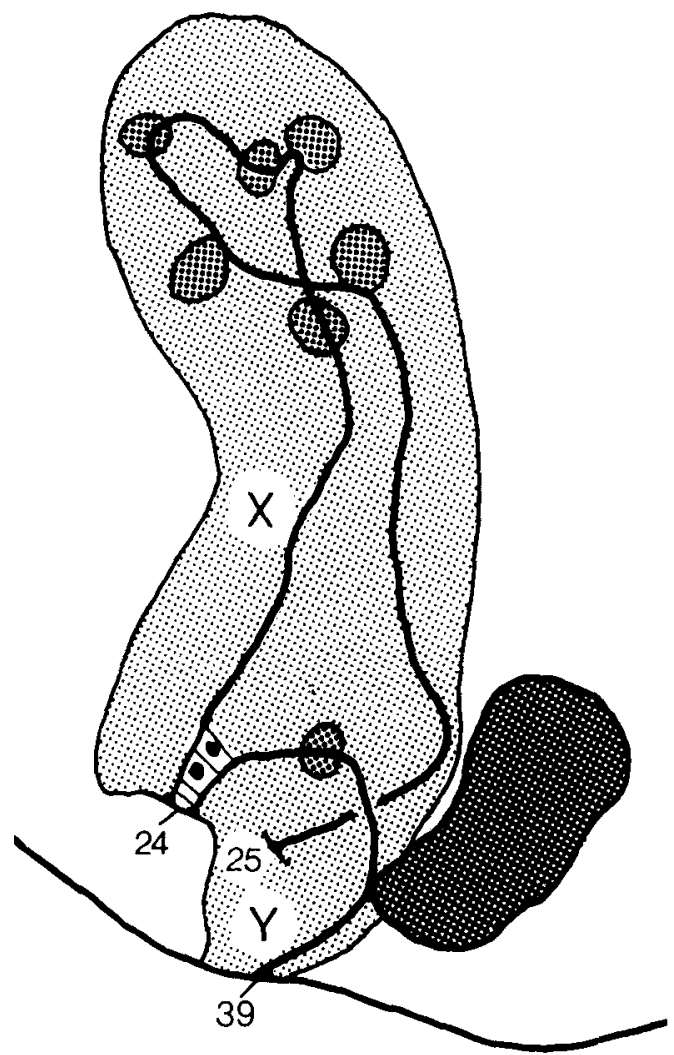




\subsubsection{Total number of recombination nodules}

The total number of recombination nodules per nucleus at early, mid and late zygotene as well as early pachytene is given in Tables II, $\mathrm{V}$ and VI. At late zygotene, the number of nodules varied from 75-161 and at early pachytene from 40-114, the mean values for the four stages being $21,60,101 \pm 25$ and $75 \pm 20$, respectively. The number of nodules per nucleus at late zygotene and early pachytene is significantly different at the $1 \%$ probability level.

At late zygotene only $72 \%$ of the complement is paired with a synaptonemal complex. The number of nodules per nucleus expected when all synaptonemal complexes have just been completed at the end of zygotene were calculated from the number observed at late zygotene assuming that all regions of the synaptonemal complex have equal possibilities of receiving a nodule (Table II). The variation in this expected number of nodules ranges from 102-215 per nucleus with a mean number of $144 \pm 32$. This number is approximately twice as large as the number of nodules present at early pachytene.

A more direct comparison of the nodule frequencies between zygotene and early pachytene was obtained by calculating the length of the synaptonemal complex per recombination nodule, the values being $2.3,2.5,1.7$ and $3.0 \mu \mathrm{m}$ of synaptonemal complex per nodule for the

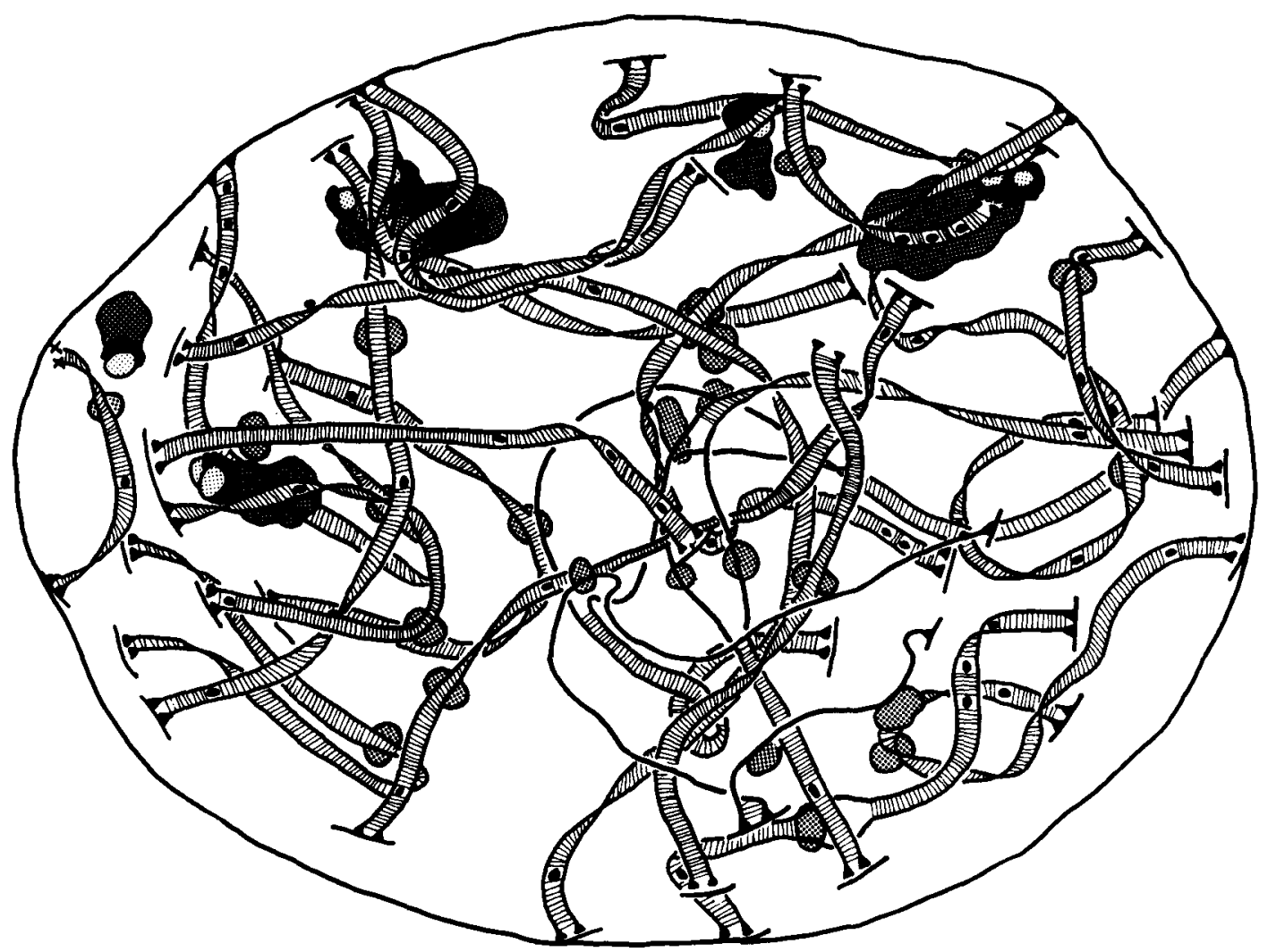

Figure 29. A complete reconstruction of an early pachytene nucleus (nucleus number 63 ). The attachment sites of the telomeres are evenly distributed throughout the nuclear envelope. In the left hand side of the reconstruction, the short arm of an acrocentric bivalent is free in the nucleoplasm (indicated by crosses). Two bivalents have unpaired middle segments each entrapping a completely paired bivalent. (Separate drawings of these interlockings are given in Figures 32 and 33.) In the lower half of the nucleus the unpaired lateral components of the secondary constriction of bivalent 9 can be seen. The $X$ and $Y$ chromosomes are terminally paired but have not yet condensed into a discrete $\mathrm{XY}$ body. 

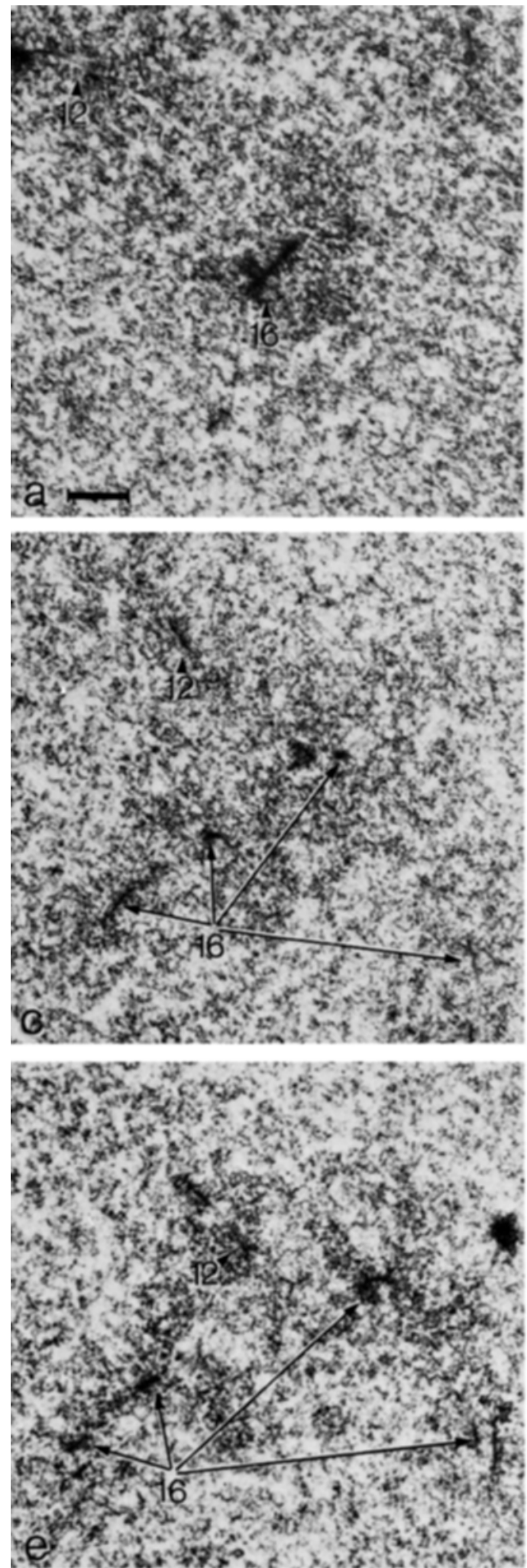
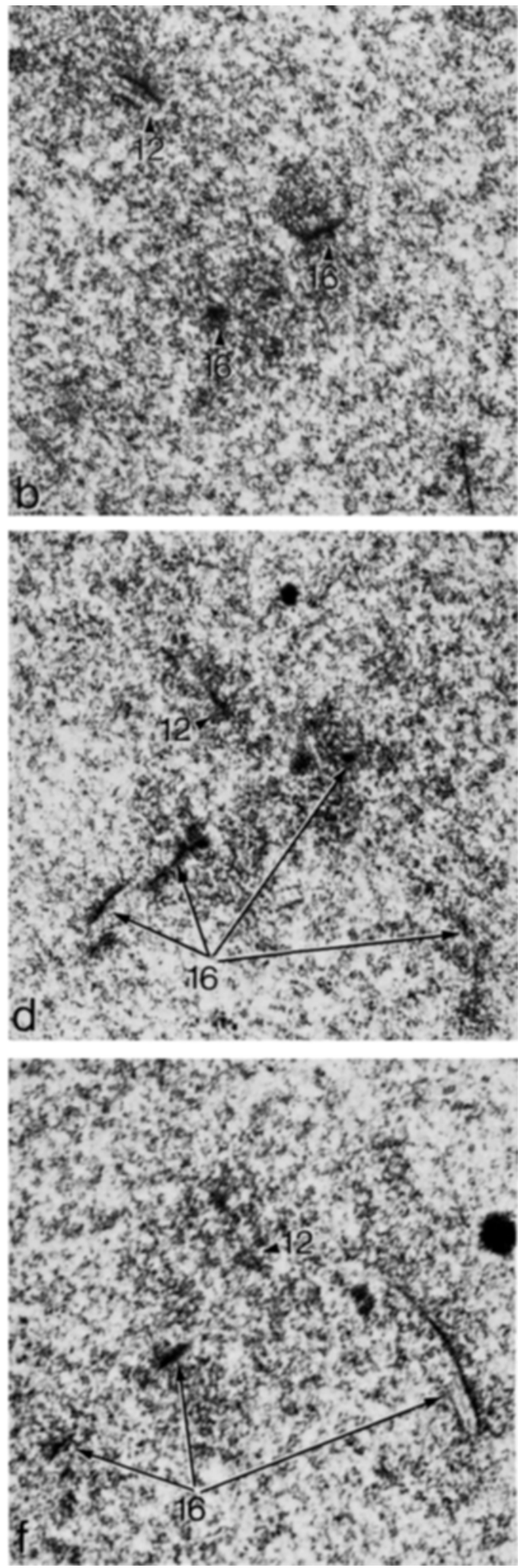

Figure 30. Electron micrographs of twelve consecutive sections showing the interlocking of bivalent 12 between the homologues of bivalent 16 in an early pachytene nucleus (nucleus number 63, Figures 29 and 32). A reconstruction of these twelve sections is shown in Figure 31. 

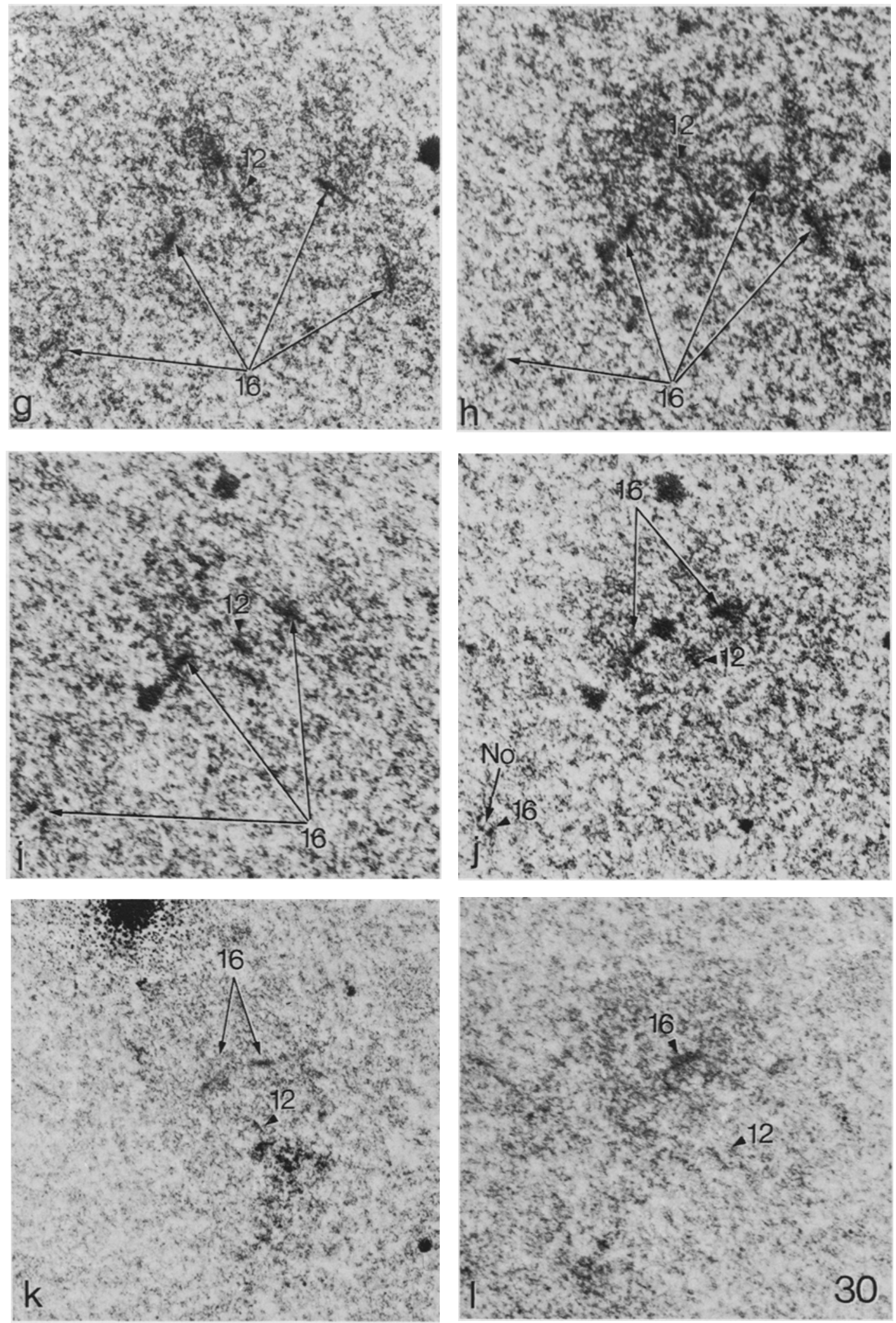


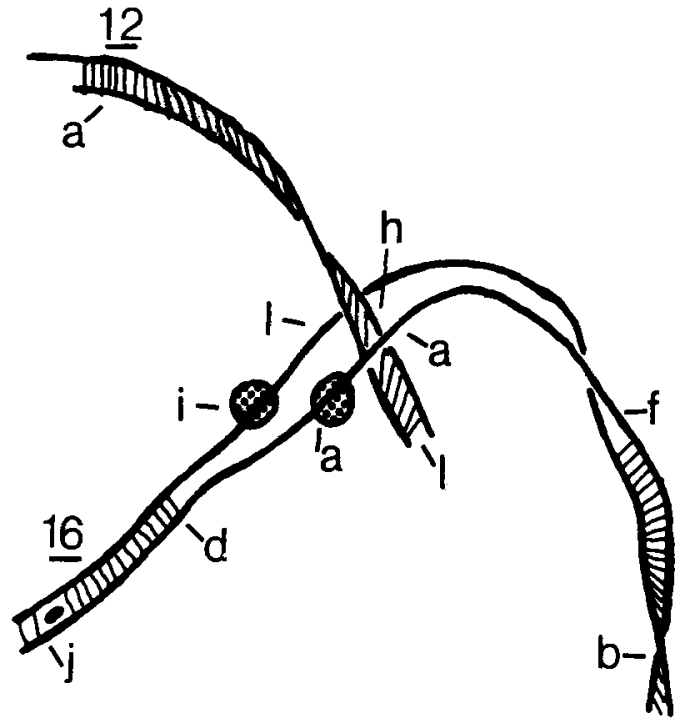

Figure 31. Reconstruction of the interlocking shown in Figure 30.

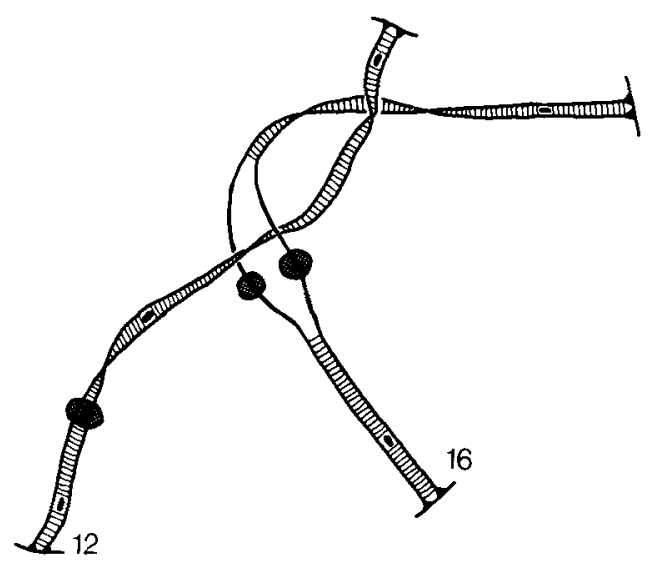

Figure 32. Reconstruction of the interlocking of bjvalent 12 between the homologues of bivalent 16 in nucleus 63 .

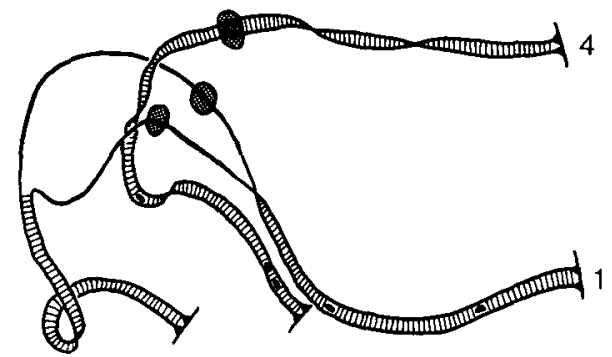

Figure 33. Reconstruction of the interlocking of bivalent 4 between the homologues of bivalent 1 in nucleus 63 . three substages of zygotene and early pachytene respectively (Table VI). The difference between late zygotene and early pachytene is significant at the $0.1 \%$ probability level.

The data in Tables II and $\mathrm{V}$ demonstrate a considerable variation in total length of synaptonemal complex per nucleus at late zygotene as well as at early pachytene. This variation is accompanied by a comparable but uncorrelated variation in the total number of recombination nodules per nucleus (Figure 40).

\subsubsection{Number of recombination nodules per bivalent}

The mean numbers of recombination nodules per bivalent at late zygotene and early pachytene are listed in Table I and IV, Table I also including the extrapolated number of nodules per bivalent at the end of zygotene. Plotting the mean synaptonemal complex lengths of a bivalent against the mean number of its observed recombination nodules in Figure 41, gives correlation between these two parameters (correlation coefficient 0.76 ) for late zygotene even though larger deviations are evident for some of

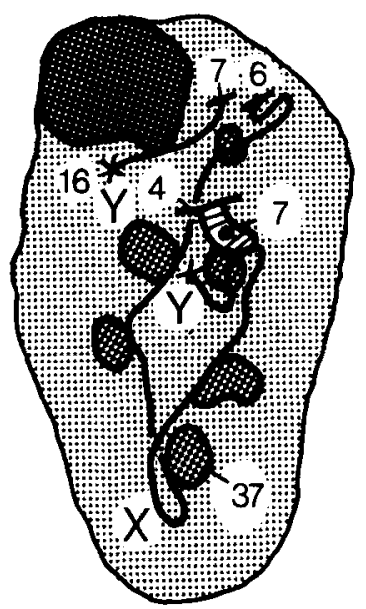

Figure 34. Reconstruction of a broken lateral component of the $Y$ chromosome from an early pachytene nucleus (nucleus number 60 ). The two free ends are denoted by crosses. One of the fragments is associated with a nucleolus-like structure and the other is paired with the $\mathrm{X}$ chromosome by a synaptonemal complex. The numbers are the section numbers. 
the bivalents (e.g., bivalents 6,7 and 8 ). The correlation between synaptonemal complex length and number of nodules per bivalent at early pachytene is high (correlation coefficient 0.96 ). The slopes of the regression lines at late zygotene and early pachytene are close being 0.46 at late zygotene and 0.38 at early pachytene.

The high correlation between synaptonemal complex length and number of recombination nodules at these two stages is also illustrated by the data in Table VII. At late zygotene there is on the average one nodule per $1.7 \mu \mathrm{m}$ of synaptonemal complex compared to $2.9 \mu \mathrm{m}$ of complex per nodule at early pachytene. A comparison of the individual bivalent arms gives a more differentiated picture. Thus, in the acrocentric bivalents the short arms at early pachytene seldom contain recombination nodules. Also the

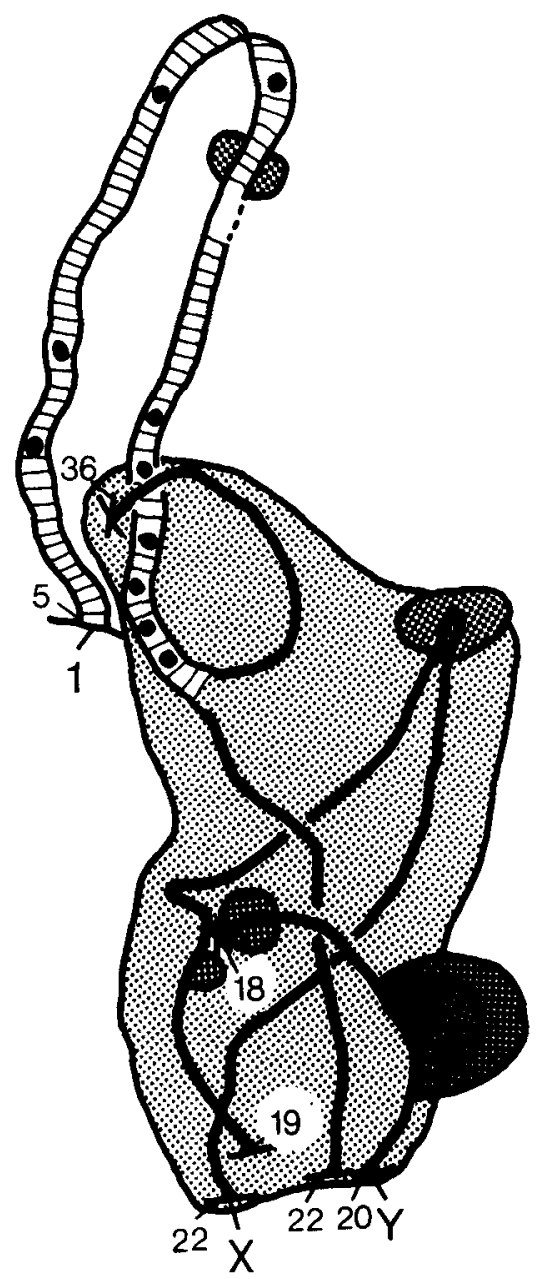

short arm of bivalent 5 has a lower number of recombination nodules at early pachytene than expected, but is should be noted that the data from this arm is from only 14 nuclei.

\subsubsection{Number of recombination nodules in the $X Y$ bivalent}

Pairing and synaptonemal complex formation in the homologous segments of the XY bivalent were found in 50 percent of the late zygotene nuclei, the paired segment containing 1 or 2 recombination nodules (Tables VIII and IX). The $\mathrm{X}$ and $\mathrm{Y}$ chromosomes were paired with a synaptonemal complex in all early pachytene nuclei. Of the $22 \mathrm{XY}$ bivalents examined at this stage, 6 were devoid of nodules, 13 were associated with one, and 3 with two nodules. As illustrated in Table VIII, the synaptonemal complex length per recombination nodule for the XY bivalent is 1.0 at late zygotene as well as at early pachytene compared to mean values of 1.7 and 3.0 for the autosomal bivalents at these stages.

\subsubsection{Bivalents without recombination nodules}

Nineteen autosomal bivalents with completed synaptonemal complexes and six $\mathrm{XY}$ bivalents were entirely devoid of nodules (Table $X)$. Three of the autosomal bivalents were from late zygotene nuclei. As shown in Table $\mathrm{X}$ a

Figure 35. Reconstruction of the broken lateral components of the $\mathrm{Y}$ chromosome and chromosome 1 in an early pachytene nucleus (nucleus number 58 ). One of the broken ends of the $Y$ chromosome (in section 18 ) is associated with the knob-like structure which marks the position of the centromere region of the $Y$ chromosome. The absence of a synaptonemal complex between this fragment of the $\mathrm{Y}$ chromosome and the $\mathrm{X}$ chromosome indicates that the homologous region of the $\mathrm{Y}$ chromosome (the short arm) has been lost. The reconstruction of bivalent 1 suggests that one of the homologues has a terminal deletion of the long arm. The unpaired regions of both homologues are embedded in the chromatin of the XY body. The deleted arm of chromosome 1 is not attached to the nuclear envelope and is $1.1 \mu \mathrm{m}$ shorter than the other arm. 
Table VI

Number of nuclei analyzed, total synaptonemal complex length, total complement length, number of recombination nodules, and synaptonemal complex length per recombination nodule.

\begin{tabular}{lccccc}
\hline Stage & $\begin{array}{c}\text { Number } \\
\text { of } \\
\text { nuclei }\end{array}$ & $\begin{array}{c}\text { Total SC } \\
\text { length } \\
(\mu \mathrm{m})\end{array}$ & $\begin{array}{c}\text { Total } \\
\text { complement } \\
\text { length }(\mu \mathrm{m})\end{array}$ & $\begin{array}{c}\text { Number } \\
\text { of } \\
\text { nodules }\end{array}$ & $\begin{array}{c}\text { SC length } \\
\text { per } \\
\text { nodule }\end{array}$ \\
\hline Early zygotene & 2 & 44.1 & - & 21 & 2.3 \\
Mid zygotene & 2 & 145.1 & - & 60 & 2.5 \\
Late zygotene & 10 & 169.0 & 234.7 & 101 & 1.7 \\
Early pachytene & 21 & $204.5^{\text {a) }}$ & $207.2^{\text {a) }}$ & 75 & 3.0 \\
Pachytene & 22 & 231 & 231 & 0 & - \\
\hline
\end{tabular}

a) The difference between the two figures is due to the two cases of interlocking.

b) Data from HOLM and RASMUSSEN (20).

complete lack of nodules is prevalent among the shorter bivalents, in particular the acrocentric bivalents of which 13 were without nodules.

\subsubsection{Distribution of recombination nodules along the bivalents}

The normalization procedure performed on each chromosome arm as outlined in section 2.4. was carried out in order to compensate for the internuclear variation in arm length before analyzing the distribution of recombination nodules along the individual bivalents. The number of nodules per bivalent segment for all nuclei at late zygotene and early pachytene is presented as histograms in Figure 42, each segment corresponding to $0.67 \mu \mathrm{m}$ of synaptonemal complex. The histograms in the left hand column compile the data from late zygotene, and those to the right the early pachytene data. The nodules appear to be evenly distributed over the chromosome arms in the paired regions of the late zygotene bivalents as well as in the fully paired early pachytene bivalents although the number of nodules per segment is considered insufficient for a reliable statistical analysis. One exception to this general trend is encountered in the short arms of the acrocentric bivalents which generally lack recombination' nodules. Recombination nodules are also less frequent at and on either side of the centromere. The synaptonemal complex length per recombination nodule is 5.2 in the two cen- tromeric segments at early pachytene, compared to a mean value of 3.0 for the autosomes, the corresponding figures for late zygotene being 3.4 and 1.7 (excluding the short arms of the acrocentric bivalents). This difference is particularly prominent in centromeric segments which include the secondary constrictions of bivalents 1,9 and 16 at early pachytene where only four nodules were found in the 21 reconstructed nuclei.

Recombination nodules tend to be more frequent in the terminal or subterminal segments of most bivalents in late zygotene as well as in early pachytene. The synaptonemal complex length per nodule is in the terminal segment 1.0 for late zygotene and 2.7 for early pachytene compared to mean values of 1.7 and 3.0 (not including the terminal segments of the short arms of the acrocentric bivalents).

\subsubsection{Nodule-nodule, nodule-telomere and nodule-centromere distances}

A more sensitive analysis of the nodule distribution on bivalent arms can be performed on the basis of the distribution of nodule-nodule, nodule-telomere and nodule-centromere distances. In order to analyze whether these distances reflect a random distribution of nodules at late zygotene and early pachytene, all distances between adjacent nodules, between each telomere and the nearest nodule, and between 


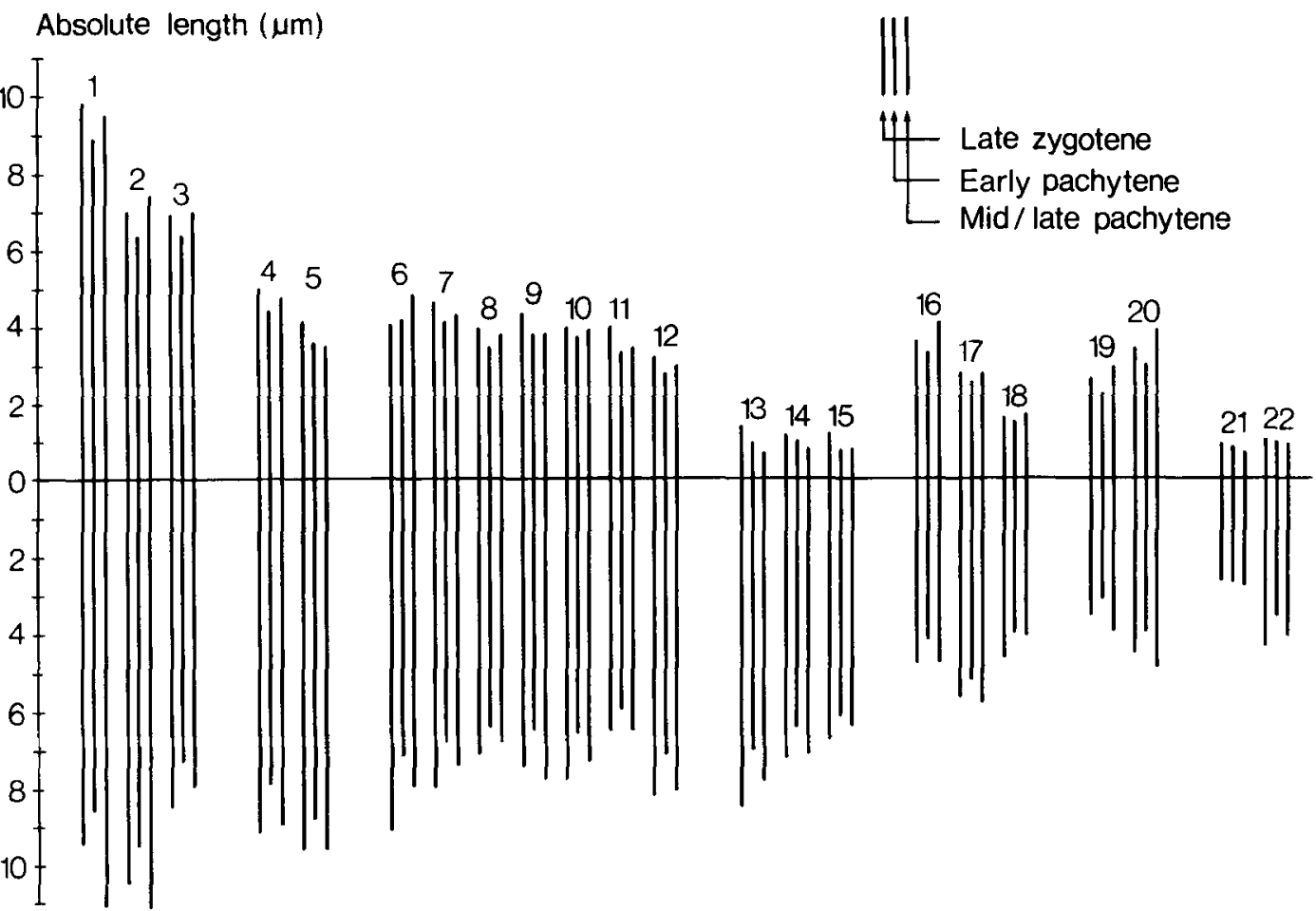

Figure 36. Idiograms of the human karyotype in late zygotene, early pachytene, and mid-late pachytene. The data for mid-late pachytene have been presented previously (20). The bivalents in early pachytene are slightly shorter than the ones in late zygotene and mid-late pachytene, see Tables I, II, IV, V. As described in section 3.3.2., bivalents 4 and 5 from mid-late pachytene should be interchanged.
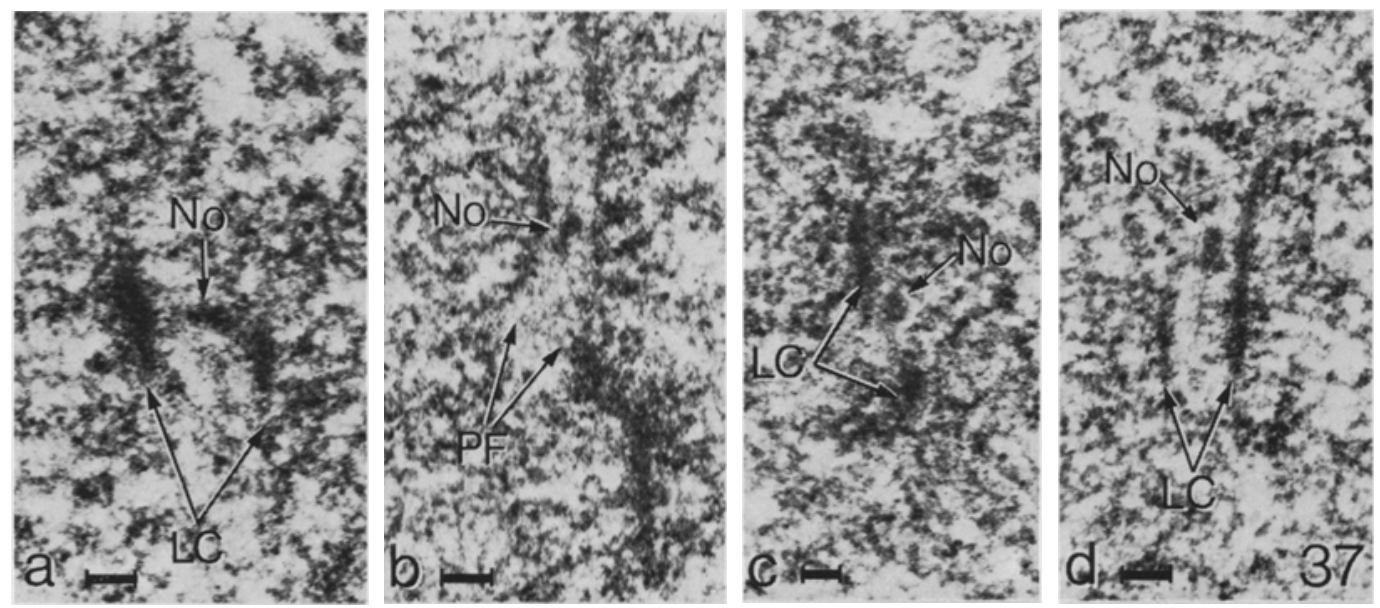

Figure 37. Four electron micrographs illustrating the ultrastructure of recombination nodules at early zygotene. Figure 37a shows two grossly aligned homologous chromosomes. The distance between the two lateral components (LC) is $200 \mathrm{~nm}$ and the central region of the synaptonemal complex is not yet fully organized. A structure resembling a recombination nodule (No) is connected to one of the lateral components. Figure $37 \mathrm{~b}$ shows a recombination nodule close to the pairing fork (PF) of two homologous chromosomes. Oblique and longitudinal sections of synaptonemal complexes with recombination nodules are illustrated in Figures $37 \mathrm{c}$ and $\mathrm{d}$. The recombination nodule shown in Figure $37 \mathrm{c}$ is positioned lateral to the plane defined by the two lateral components. $(\mathrm{Bar}=0.1 \mu \mathrm{m})$ 

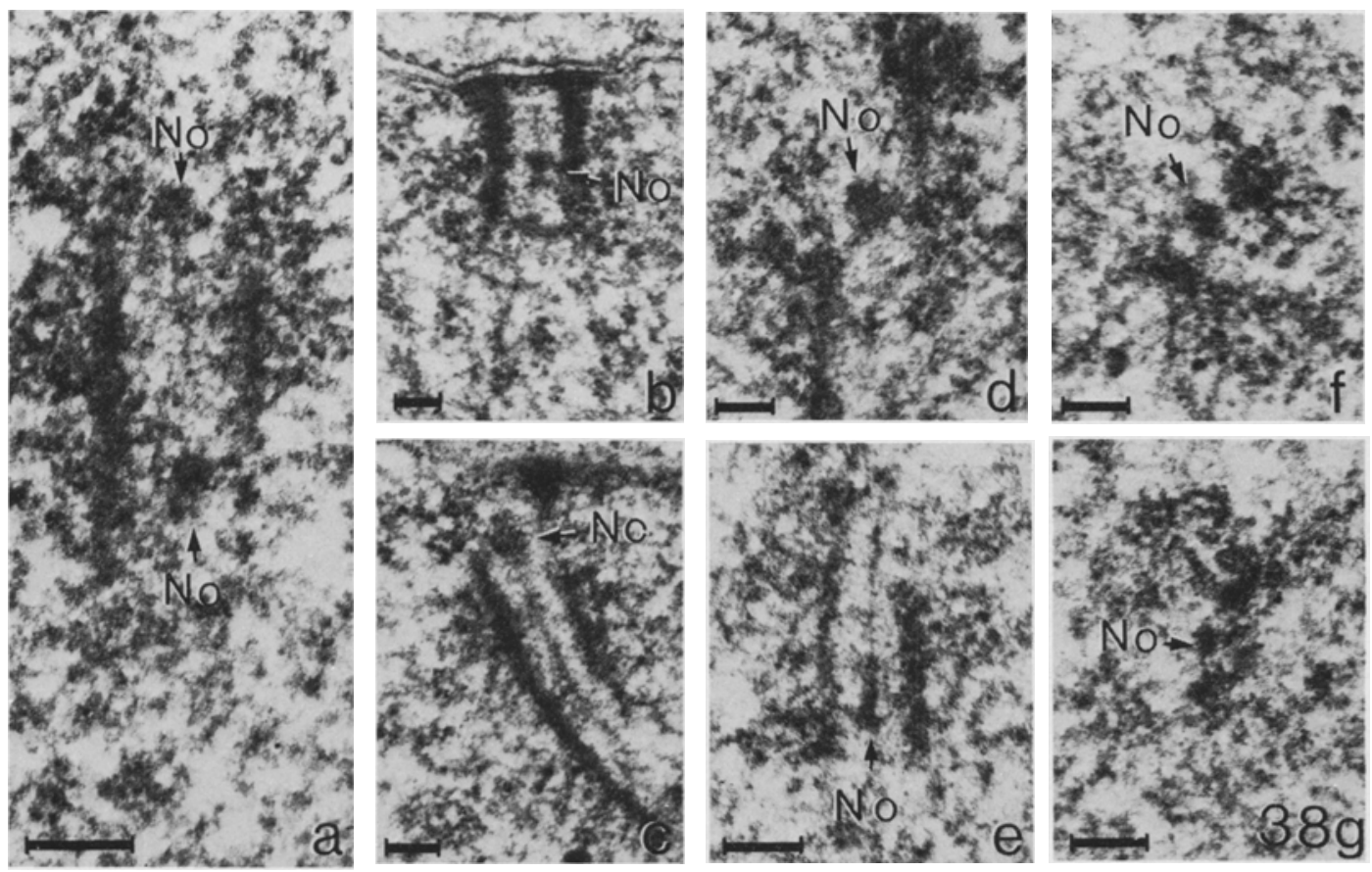

Figure 38. Ultrastructure of recombination nodules at late zygotene. Longitudinal sections of synaptonemal complexes with nodules ( $\mathrm{No}$ ) are shown in Figures $38 \mathrm{a}, \mathrm{d}$, and e. Figures $38 \mathrm{~b}$ and $\mathrm{c}$ illustrate recombination nodules lying close to the telomere of the bivalent. The transverse sections of the synaptonemal complex seen in Figures $38 \mathrm{f}$ and $\mathrm{g}$ show recombination nodules lying close to the plane defined by the two lateral components. Note the fibrillar connections between the lateral components and the recombination nodules. ( $\mathrm{Bar}=0.1 \mu \mathrm{m}$ )

each centromere and the nearest nodule were measured. The distances for late zygotene and early pachytene were divided into $0.1 \mu \mathrm{m}$ classes and the relative frequencies were calculated for each class as illustrated in Figure 43. For comparison a theoretical distribution of nodule-nodule, nodule-telomere and nodule-centromere distances (shown by the dots in Figure 43) was designed in the following way: For early pachytene the mean absolute lengths and centromere indices of the 22 autosomal bivalents were fed into a Honeywell Bull $\mathrm{H} 6000 \mathrm{com}$ puter which was programmed to position the nodules at random along the bivalents. The mean diameter of the recombination nodule was defined to be $80 \mathrm{~nm}$ which thus equals the minimum center to center distance of two individual nodules. The minimum distance from the center of a nodule to a telomere or a centromere is accordingly $40 \mathrm{~nm}$. The computer was programmed to position one nodule at a time and in those cases where the nodule position generated by the computer was closer than 80 $\mathrm{nm}$ to a previously positioned nodule or closer than $40 \mathrm{~nm}$ to a telomere or a centromere a new position was generated. The program was run 3000 times (which equals 3000 "average" nuclei) and the nodule-nodule, nodule-telomere and nodule-centromere distances were pooled separately, divided into $0.1 \mu \mathrm{m}$ classes and the relative frequencies calculated for each class. These ditributions thus illustrate the type of distance distribution which will result if recombination nodules associate with the synaptonemal complex at random.

The theoretical distance distributions for late zygotene were obtained in a similar way using the mean values for the length of the synaptonemal complex of the bivalents, mean centromere indices and mean number of nodules 

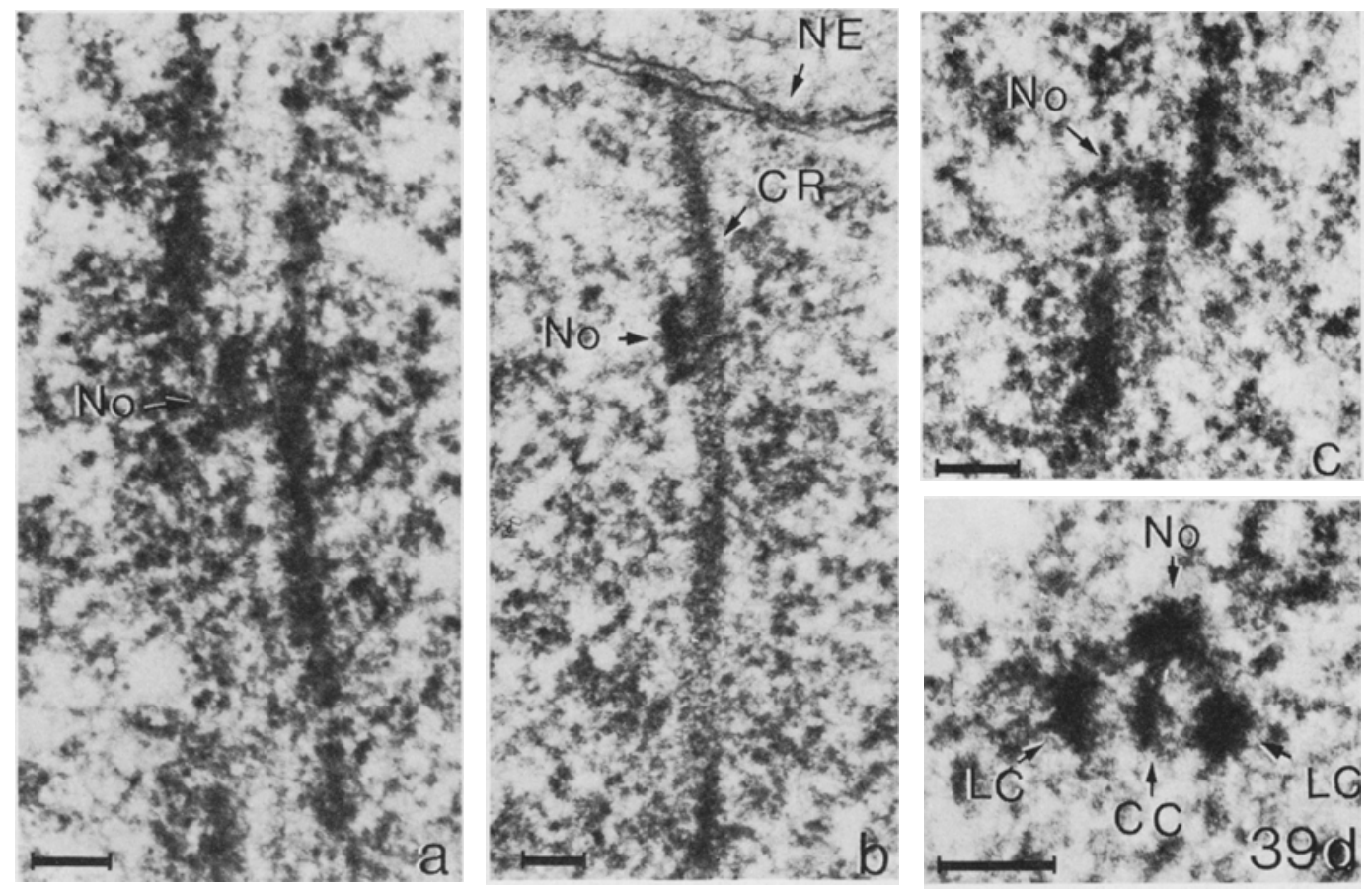

Figure 39. Ultrastructure of recombination nodules at early pachytene. Longitudinal sections of synaptonemal complexes with recombination nodules (No) are shown in Figures 39a-c and a transverse section in Figure 39d. Only the central region (CR) of the synaptonemal complex is present in Figure 39b. The recombination nodule is intimately associated with the central region and is positioned lateral to this. Figure $39 \mathrm{~d}$ also demonstrates a recombination nodule located outside the synaptonemal complex and connected to the lateral component (LC) as well as the central component $(\mathrm{CC})$. NE, nuclear envelope $(\mathrm{Bar}=0.1 \mu \mathrm{m})$
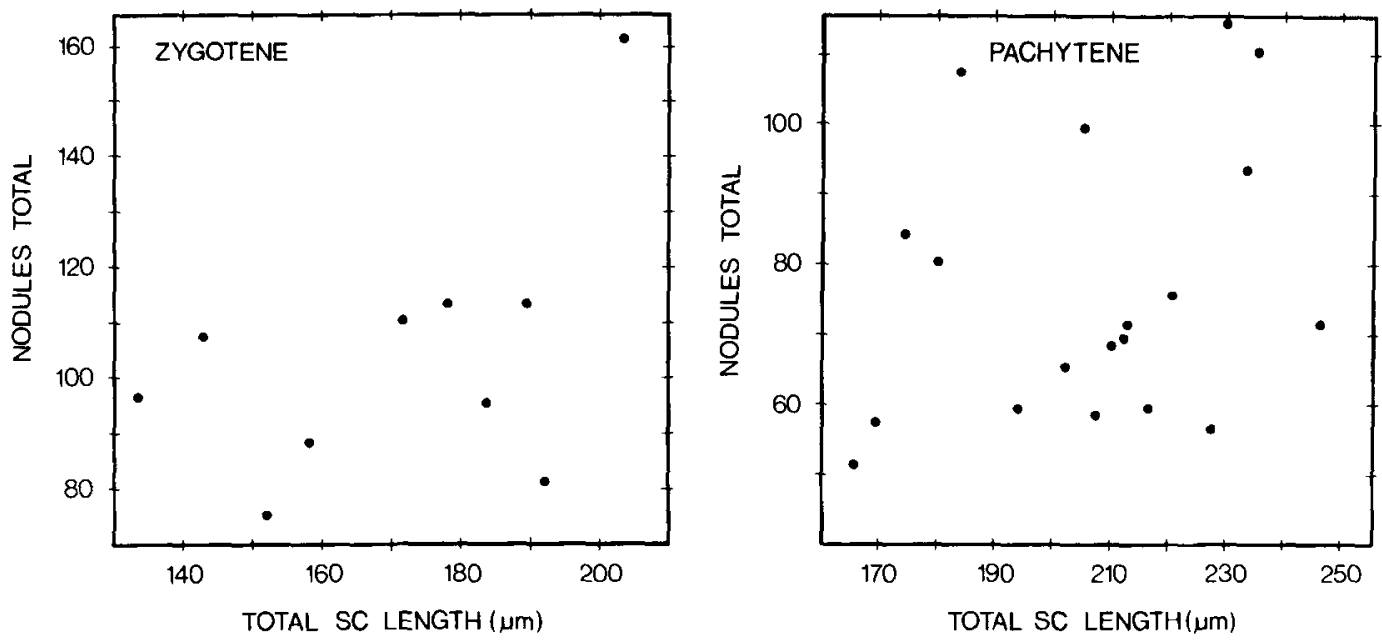

Figure 40. The total length of the autosomal synaptonemal complexes in 10 late zygotene nuclei and 21 early pachytene nuclei is plotted against the total number of autosomal recombination nodules found in the nucleus. No correlation is observed. 

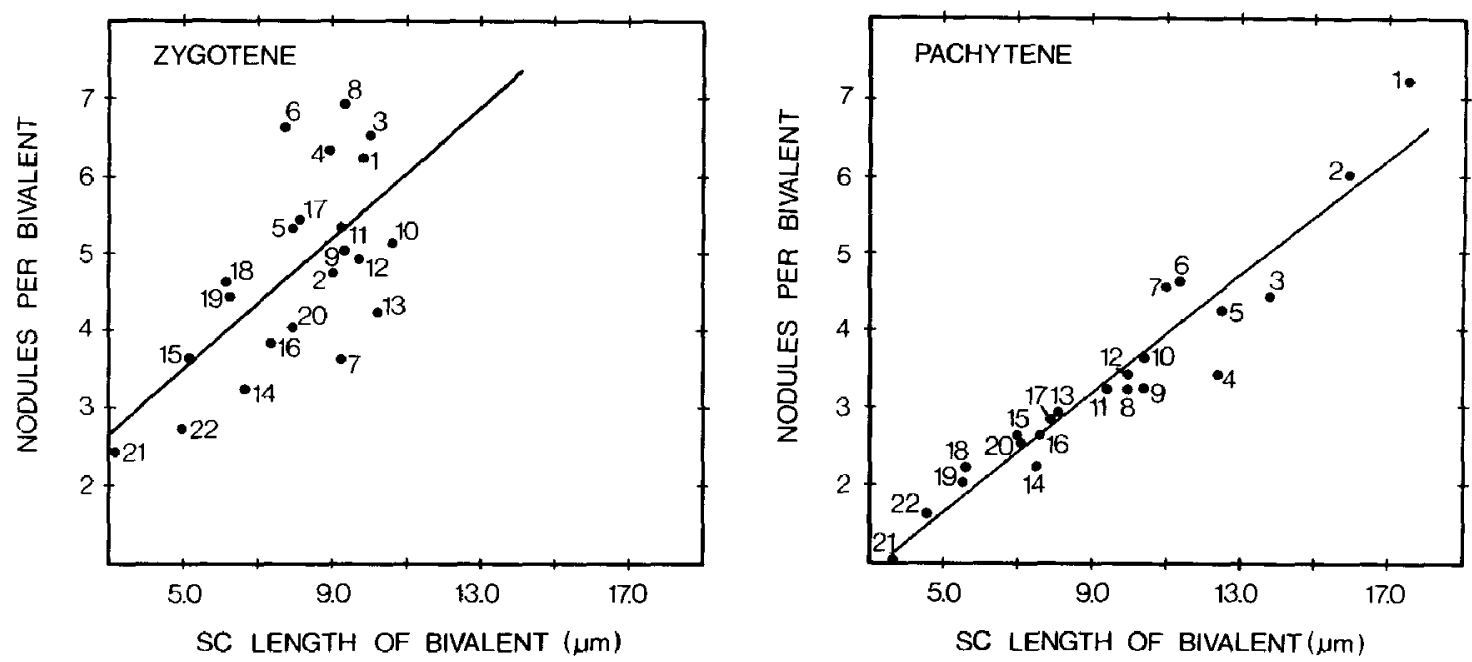

Figure 41 . The mean length of the synaptonemal complexes for the individual bivalents in 10 zygotene and 21 early pachytene nuclei is plotted against the mean number of recombination nodules per bivalent. The correlation coefficient for late zygotene is 0.76 and for early pachytene 0.96 .

\section{Table VII}

Synaptonemal complex length $(\mu \mathrm{m})$ per recombination nodule for the long and the short arms of the 22 autosomal bivalents. The data are the mean values of 10 zygotene and 21 early pachytene nuclei respectively.

\begin{tabular}{|c|c|c|c|c|c|c|}
\hline \multirow{2}{*}{$\begin{array}{l}\text { Bivalent } \\
\text { number }\end{array}$} & \multicolumn{6}{|c|}{ Synaptonemal complex $(\mu \mathrm{m})$ per recombination nodule } \\
\hline & $\begin{array}{l}\text { Long arm } \\
\text { Zygotene }\end{array}$ & $\begin{array}{l}\text { Long arm } \\
\text { Pachytene }\end{array}$ & $\begin{array}{l}\text { Short arm } \\
\text { Zygotene }\end{array}$ & $\begin{array}{l}\text { Short arm } \\
\text { Pachytene }\end{array}$ & $\begin{array}{c}\text { Entire } \\
\text { bivalent } \\
\text { Zygotene }\end{array}$ & $\begin{array}{c}\text { Entire } \\
\text { bivalent } \\
\text { Pachytene }\end{array}$ \\
\hline 1 & 1.8 & 2.4 & 1.4 & 2.2 & 1.6 & 2.3 \\
\hline 2 & 2.0 & 2.5 & 1.9 & 3.2 & 1.9 & 2.7 \\
\hline 3 & 1.6 & 3.0 & 1.5 & 3.2 & 1.6 & 3.1 \\
\hline 4 & 1.9 & 3.6 & 1.5 & 3.7 & 1.7 & 3.7 \\
\hline 5 & 1.4 & 2.6 & 1.6 & $5.8^{\mathrm{a})}$ & 1.5 & 2.8 \\
\hline 6 & 1.4 & 2.1 & 1.0 & 3.5 & 1.2 & 2.5 \\
\hline 7 & 2.2 & 2.2 & 2.6 & 3.2 & 2.5 & 2.5 \\
\hline 8 & 1.5 & 2.7 & 1.1 & 3.9 & 1.3 & 3.1 \\
\hline 9 & 1.9 & 2.9 & 1.8 & 3.9 & 1.8 & 3.1 \\
\hline 10 & 2.8 & 3.3 & 1.5 & 2.2 & 2.1 & 2.8 \\
\hline 11 & 2.0 & 2.8 & 1.4 & 3.0 & 1.7 & 2.8 \\
\hline 12 & 2.5 & 3.2 & 1.4 & 2.8 & 2.0 & 3.0 \\
\hline 13 & 1.8 & 2.5 & 5.0 & 19.0 & 1.9 & 2.8 \\
\hline 14 & 1.7 & 3.1 & - & - & 1.8 & 3.5 \\
\hline 15 & 1.4 & 2.5 & - & 8.0 & 1.4 & 2.7 \\
\hline 16 & 2.2 & 3.2 & 1.7 & 2.4 & 1.9 & 2.8 \\
\hline 17 & 1.9 & 2.9 & 1.0 & 2.6 & 1.5 & 2.8 \\
\hline 18 & 1.4 & 2.7 & 1.2 & 2.1 & 1.4 & 2.5 \\
\hline 19 & 1.4 & 2.7 & 1.4 & 3.0 & 1.4 & 2.8 \\
\hline 20 & 1.9 & 3.1 & 2.1 & 2.4 & 2.0 & 2.8 \\
\hline 21 & 1.1 & 2.7 & 6.0 & 17.0 & 1.3 & 3.5 \\
\hline 22 & 1.6 & 2.2 & 7.0 & $15.0^{\text {a) }}$ & 1.8 & 2.3 \\
\hline Mean & 1.8 & 2.8 & $1.5^{\mathrm{b})}$ & $3.1^{\mathrm{b})}$ & 1.7 & 2.9 \\
\hline Stand. dev. & 0.4 & 0.4 & 0.4 & 0.9 & 0.3 & 0.4 \\
\hline
\end{tabular}

a) 14 nuclei from normal males

b) The short arms for the acrocentric bivalents are not included. 
Table VIII

Absolute length of the $X$ and $Y$ chromosomes, synaptonemal complex length of their paired segment, and synaptonemal complex length per recombination nodule.

\begin{tabular}{lcccccccc}
\hline Stage & $\begin{array}{c}\text { Number } \\
\text { of } \\
\text { nuclei }\end{array}$ & $\begin{array}{c}\text { Length } \\
\text { of } \mathrm{X} \\
(\mu \mathrm{m})\end{array}$ & $\begin{array}{c}\text { Standard } \\
\text { deviation }\end{array}$ & $\begin{array}{c}\text { Length } \\
\text { of } \mathrm{Y} \\
(\mu \mathrm{m})\end{array}$ & $\begin{array}{c}\text { Standard } \\
\text { deviation }\end{array}$ & $\begin{array}{c}\text { SC length } \\
(\mu \mathrm{m}) \text { of } \\
\text { paired } \\
\text { segment }\end{array}$ & $\begin{array}{c}\text { Standard SC length } \\
\text { devion }\end{array}$ & $\begin{array}{c}(\mu \mathrm{m}) \text { per } \\
\text { nodule }\end{array}$ \\
\hline Late zygotene & 10 & 11.8 & 1.9 & 4.3 & 0.7 & 0.6 & 0.1 & 1.0 \\
Early pachytene & 22 & 10.5 & 1.7 & 3.6 & 0.7 & 0.9 & 0.4 & 1.0 \\
\hline
\end{tabular}

per nucleus. In most bivalents, however, the centromere region was unpaired (see Figure 12) and the unpaired regions were "excluded" by joining the mean paired lengths of the two arms of each partially paired bivalent. The joined pairing forks were then used as a centromere. This approximation is considered to have only a minor effect on the theoretical distribution resulting in a slight underrepresentation of the very long distances (distances longer than the mean paired length of a bivalent arm) and consequently a slight overrepresentation of all other distances.

A comparison between the observed and theoretical distribution of nodule-nodule distances in late zygotene clearly shows that nodules are not positioned at random with respect to each other. Twenty-seven percent of the nodule-nodule distances are in the interval from 0.2-0.4 $\mu \mathrm{m}$ whereas only 16 percent are expected if nodules are positioned randomly. In the range from $1.0-2.0 \mu \mathrm{m}$, the reverse pattern is observed, the observed distribution revealing fewer distances than expected. Finally, it should be noted that a critical minimum nodule-nodule distance is not revealed in the pre-

\section{Table IX}

Number of XY bivalents with 0,1 , and 2 recombination nodules.

\begin{tabular}{llcc}
\hline Stage & \multicolumn{3}{c}{ Number of bivalents with } \\
& 0 & $\begin{array}{c}1 \\
\text { nodules }\end{array}$ & 2 \\
\hline Late zygotene & $5^{\text {a) }}$ & 4 & 1 \\
Early pachytene & 6 & 13 & 3 \\
\hline
\end{tabular}

a) $X$ and $Y$ not paired
Table X

Bivalents with completed synaptonemal complex and without recombination nodules.

\begin{tabular}{ccc}
\hline $\begin{array}{c}\text { Bivalent } \\
\text { number }\end{array}$ & $\begin{array}{c}\text { Number of } \\
\text { nuclei at } \\
\text { late zygotene }\end{array}$ & $\begin{array}{c}\text { Number of } \\
\text { nuclei at } \\
\text { early pachytene }\end{array}$ \\
\hline 4 & - & 2 \\
5 & - & 1 \\
7 & - & 1 \\
13 & - & 2 \\
14 & - & 1 \\
15 & - & 2 \\
18 & - & 1 \\
20 & - & 1 \\
21 & 2 & 5 \\
22 & 1 & 3 \\
$X Y$ & - & 6 \\
\hline
\end{tabular}

sent analysis, the shortest distances being equal to the diameter of the nodules.

At early pachytene the observed distribution of nodule-nodule distances is in good agreement with the expected distribution for all distances above $0.6 \mu \mathrm{m}$. The high number of distances between 0.4 and $0.5 \mu \mathrm{m}$ probably reflects a nonsignificant variation due to the relatively low number of nuclei, whereas the pronounced decrease in the frequency of the shorter distances in the range between 0.1 and $0.4 \mu \mathrm{m}$ reflects a real decrease. Only $10 \%$ of all distances is present in this interval compared to an expected value of $18 \%$. It appears as if this deficit of shorter distances is distributed evenly among the longer distances (see Figure 42d).

The expected and observed distributions for the nodule-telomere distances also exhibit considerable differences. At late zygotene the dis- 

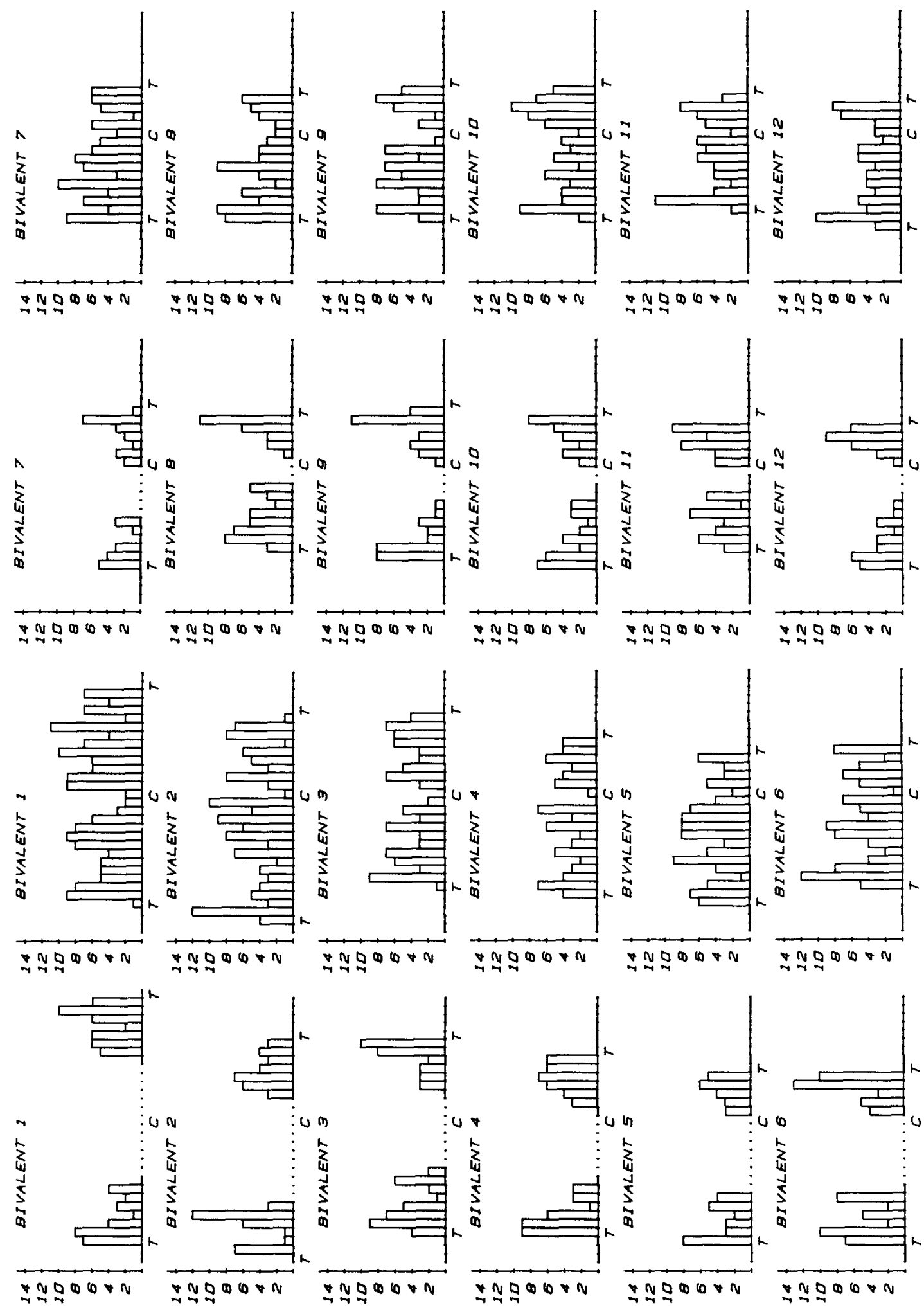

Figure 42. Histograms illustrating the distribution of recombination nodules along the individual bivalents at late zygotene (left column) and early pachytene (right column). The mean lengths of the bivalents are shown on the $\mathrm{X}$-axis with $\mathrm{T}$ and $\mathrm{C}$ denoting the position of the telomeres and the centromere, respectively. The short arm is positioned to the right and the long arm to the left, each divided into $0.67 \mu \mathrm{m}$ intervals. The Y-axis shows the number of nodules found in each segment in the 10 late zygotene nuclei and the 21 early pachytene nuclei.

The distribution of nodules at late zygotene is only given for the intervals where 5 or more of the 10 bivalents are 

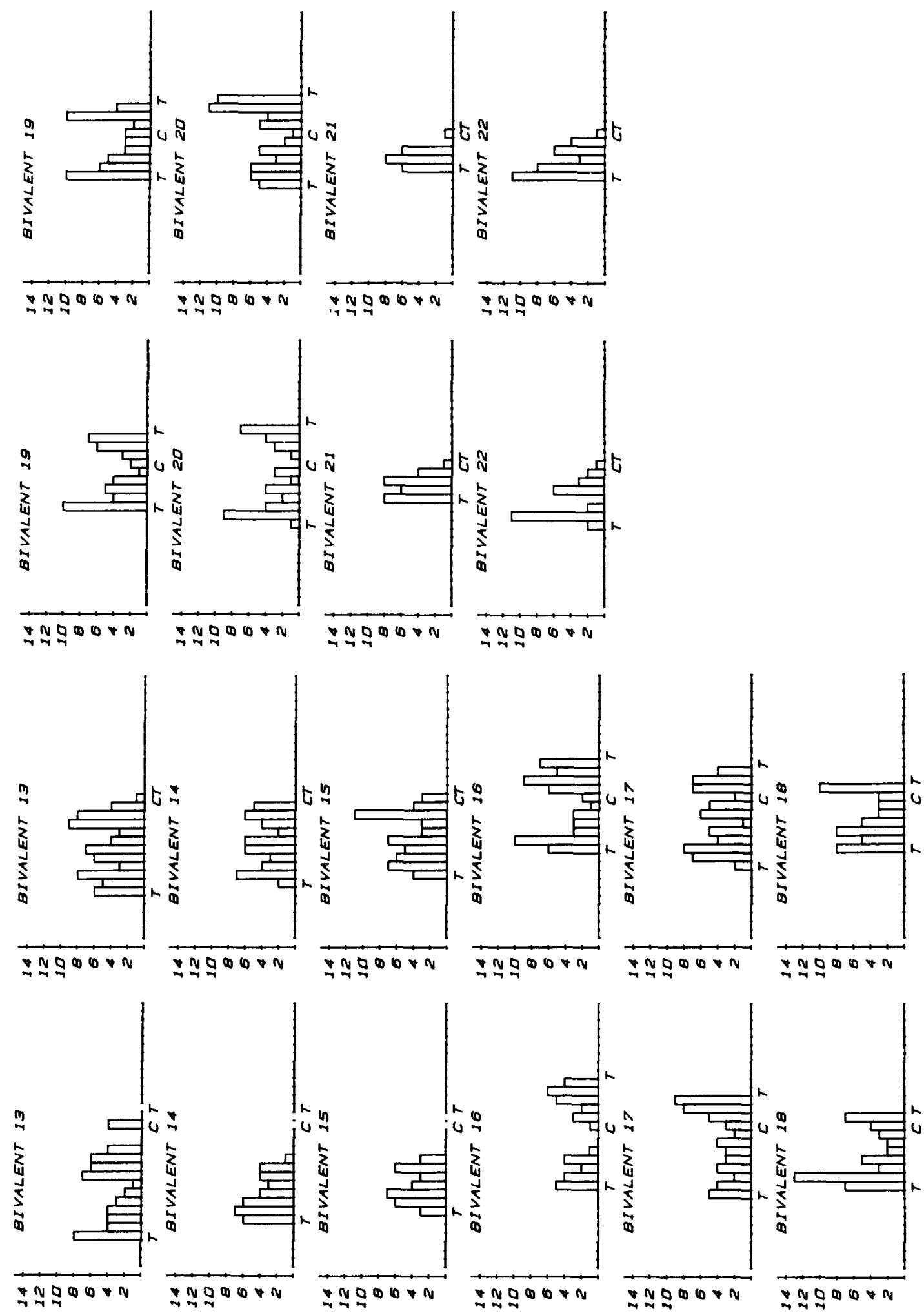

paired. In these intervals the number of nodules per segment has been corrected for the degree of pairing, thus giving the number of nodules expected, when the segments in all nuclei have completed synaptonemal complex formation. The number of nodules in a given segment was divided by the number of nuclei where the segment is paired and the value was then multiplied by 10 . The distribution of nodules in the short arms of bivalents 5 and 22 in pachytene is corrected in an analogous way after leaving out the 7 nuclei with the $t(5,22)$ translocation. 

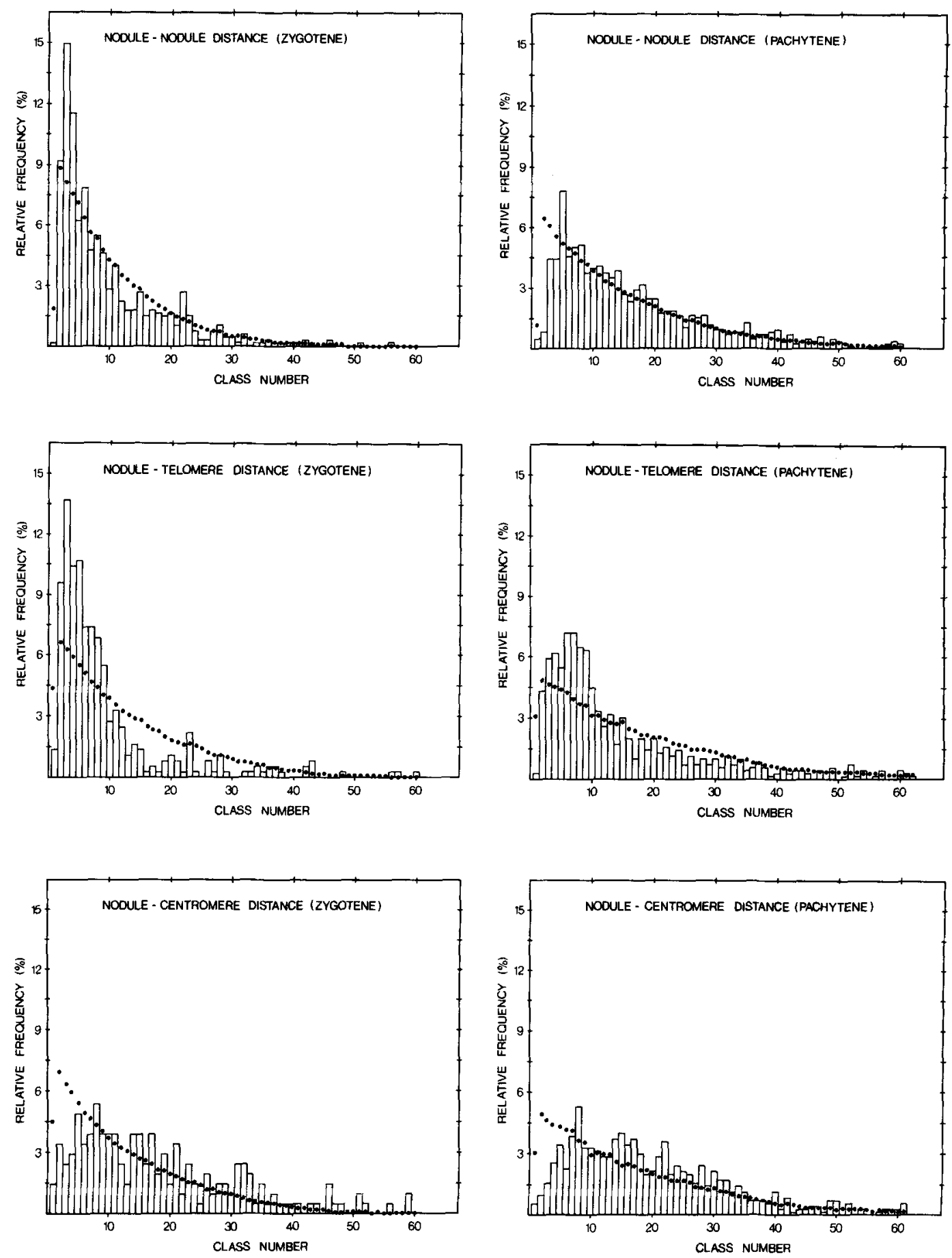

Figure 43. Histograms showing the distribution of nodule-nodule, nodule-telomere, and nodule-centromere distances expressed as relative frequencies of distances in $0.1 \mu \mathrm{m}$ classes. $(n=677,365,205$ and 859, 696, 700 for the three types of distances at zygotene and pachytene respectively). The dotted lines are theoretical destributions assuming a random distribution of recombination nodules on bivalent arms. ( $n=201,989 ; 110,011 ; 110,011$ and 125,$905 ; 99,095 ; 99,095$ for the three types of distances at zygotene and pachytene respectively). For further details see section 3.7.7. 
tances from 0.1 to $0.9 \mu \mathrm{m}$ represent $71 \%$ of all distances compared to an expected value of $43 \%$, whereas the reverse pattern is evident for the distances above $0.9 \mu \mathrm{m}$. A similar but less pronounced deviation between the observed and the expected distribution is evident in early pachytene where the distances in the range from 0.1 to $0.9 \mu \mathrm{m}$ account for $49 \%$ of all distances, while only $34 \%$ is expected were the nodules positioned randomly with respect to the telomere. These results confirm the observed prevalence of nodules in the telomere region revealed by the nodule distribution along the bivalent illustrated in the histograms in Figure 41 and discussed in section 3.7.6.

The observed distribution of nodule-centromere distances clearly differs from the distribution of nodule-nodule distances as well as from the distribution of nodule-telomere distances. When compared to the theoretical random distribution a deficit of shorter distances is observed in the range from 0.1 to 1.0 $\mu \mathrm{m}$ while distances longer than $1.0 \mu \mathrm{m}$ are in excess. It should be noted that the distribution of nodule-centromere distances appears very similar in late zygotene and in early pachytene. These results are in agreement with the low number of recombination nodules in the centromere region revealed by the analysis of the distribution of nodules along the bivalents (see section 3.7.6.).

\section{DISCUSSION}

\subsection{Chromosome pairing}

\subsubsection{General aspects}

The individual phases of chromosome pairing and synaptonemal complex formation in human spermatocyte nuclei are essentially identical to the sequence of events described in a number of organisms $(12,14,15,19,32,40,52,53)$. The organization of lateral components preferentially starts from the telomere and is followed by an association of the lateral component of the telomere region to the nuclear envelope, the attachment sites being distributed evenly over the inner nuclear membrane. Subsequently, the attachment sites become concentrated within a limited region of the nuclear envelope giving rise to a typical chromosome bouquet.
The combined effect of these events is an increased probability for a juxtaposition of homologous telomere regions thereby initiating the intimate pairing with synaptonemal complex formation. In contrast to the situation in for example Sordaria (53), Lilium (19) and maize (14) but in harmony with that of Bombyx (40) synaptonemal complex formation is almost exclusively initiated from the attached telomere regions and in late zygotene synaptonemal complex formation is a rule progressing from both ends of the bivalents. Chromosome pairing is highly asynchronous within a nucleus and in many zygotene nuclei completely paired bivalents with continuous synaptonemal complexes and chromosomes with incomplete lateral components were present concurrently. The analysis of chromosome pairing in 10 late zygotene nuclei (see Figure 12) seems to indicate that the shorter, nonacrocentric chromosomes are the first to pair possibly because the telomere movements on the nuclear membrane which is a prerequisite for the alignment of homologous telomeres are less impeded by the physical size of the chromosomes than those of the longer chromosomes. A similar intranuclear asynchrony with an early pairing of shorter chromosomes has been described in Sordaria (53). The effect of physical restrictions to chromosome movements is in particular pronounced for the acrocentric chromosomes where the short arms carry the nucleolus organizing regions and generally a nucleolus. These short arms are as a rule the last to pair and it appears that pairing of these arms is seldom initiated from their attachment sites but is the result of pairing initiated at the opposite end and progressing across the centromere region (Figure 12). Late pairing of nucleolus organizing regions has previously been demonstrated for maize (14).

Whether the presence of the differential segments on the $\mathrm{X}$ and $\mathrm{Y}$ chromosomes imposes similar constraint on the pairing of these chromosomes is unknown, but late pairing of sex chromosomes may be common since a similar delay in pairing and synaptonemal complex formation was reported for Chinese hamster (36).

It is the general impression from the present study that the pairing by the synaptonemal 
complex is highly precise, only involving homologous chromosomes and with a precise matching of homologous regions along the synaptonemal complex of the individual bivalent. This observation has gained further support by the study of a balanced translocation, where at early pachytene a translocation quadrivalent is invariably formed. At later stages of pachytene nonhomologous pairing was noted on two occasions (22).

It can accordingly be concluded that in human spermatocytes the stage of homologous pairing coincides with the stage where recombination nodules are present. The frequent absence of pairing with a synaptonemal complex in the secondary constrictions of bivalents 1 and 9 may imply that these regions do not fullfil the requirement for homology.

In situ hybridization studies have demonstrated the enrichment with highly repetitive satellite DNA's of these segments (16), and they are known to display considerable morphological variability. As has been argued for repeated ribosomal DNA sequences in maize (14) it is accordingly very unlikely that repetitive DNA plays any role in chromosome pairing in human spermatocytes. Furthermore it has been demonstrated that the DNA break-repair activities believed to signify recombinational events during pachytene are absent in satellite DNA of human spermatocytes (23).

\subsubsection{Interlocking}

The presence of irregularities such as interlockings and chromosome or bivalent breaks in 8 out of 10 late zygotene nuclei is in striking contrast to the highly regular chromosome pairing at pachytene seen in human spermatocytes (20) as well as in many other organisms (see for example 1, 3, 11, 12, 15, 40, 53). In the human spermatocytes analyzed here, $60 \%$ of the late zygotene nuclei contains one or more intact or resolving interlocking compared to values of $4.8 \%$ for early pachytene and $9.5 \%$ for mid-late pachytene. It thus appears that interlockings regularly occur during chromosome pairing in zygotene but that most of the interlockings disappear between late zygotene and early pachytene. Although the possibility that these nuclei degenerate before pachytene cannot be ruled out, the high frequency of irregularities speaks against an elimination by nuclear degeneration.

This contention is supported by the investigation of diploid Bombyx oocytes where interlockings were frequent at late zygotene but absent at pachytene (40). Three dimensional reconstructions of late zygotene nuclei of other organisms have not revealed interlockings (15, 53). In Lilium (19), indications of interlockings were, however, found in the form of closely associated nonhomologous chromosomes with discontinuous lateral components.

Interlocked chromosomes and bivalents have been reported from diplotene to metaphase $I$ in light microscopical studies but never at frequencies comparable to those of late zygotene nuclei in Bombyx oocytes and human spermatocytes $(2,9,31$, see 5 for review). This discrepancy may in part be attributed to the fact that the preservation of an interlocking until diplotene-metaphase I depends upon the presence of a chiasma on both sides of the region of interlocking but may also be due to a mechanism which can resolve the interlockings.

It has repeatedly been postulated that in some organisms such as wheat (7), Locusta (2) and maize $(29,30)$ the primary alignment of homologous chromosomes occurs during or immediately after the last premeiotic mitosis, i.e., prior to leptotene, and that this premeiotic alignment of the homologous may prevent interlocking as well as multivalent formation in polyploid organisms (43). This is certainly not the case in those organisms where leptotene and zygotene nuclei have been reconstructed. A number of investigations including the present one have unequivocally demonstrated that homologous chromosomes are either distributed in different regions of the nucleus prior to the initiation of pairing at leptotene $(14,19$, $40,48)$ or even located in separate nuclei $(28$, $49,53)$.

\subsubsection{Correction of interlocking}

There are three possible ways by which interlockings can resolve: 1) by release of a bivalent from the nuclear envelope followed by a withdrawal of the interlocked bivalent through the 
unpaired segment of the interlocking bivalent. (This possibility will only apply to bivalent interlockings.) 2) by dissolution of the synaptonemal complex to the attachment site on the nuclear envelope followed by movements of the interlocked bivalent or chromosomes relative to the interlocking bivalent until they are untangled. Subsequently, unpaired lateral components are re-paired by reformation of synaptonemal complexes. (This type of correction may only be possible in bivalent regions where crossing over has not or not yet occurred.) 3) by breakage and rejoining of one or both of the interlocked chromosomes or bivalents.

The first of these possibilities, that the telomeres of a bivalent may detach from the nuclear envelope in order to become released from an interlocking has been inferred from observations in Lilium (19) but lacks observational support in human spermatocytes as well as in Bombyx oocytes (40).

In the present study strong evidence has been obtained in favor of a resolution of interlockings by breakage and rejoining of chromosomes or bivalents. Most of the breaks of the lateral components and synaptonemal complexes observed at late zygotene were in chromosomes or bivalents which clearly appear to have been interlocked by other bivalents (see for example Figures 14, 17 and 18) and in one case, one of the fragments of a broken chromosome was still located between an unpaired segment of another bivalent (Figure 17). The general absence of breaks at later stages (20) then indicates that the late zygotene breaks are repaired by rejoining of the two fragments.

Evidence in favor of the second alternative, resolution of interlockings by dissolution and reassembly of the synaptonemal complex, has been obtained in diploid Bombyx oocytes (40) where crossing over is lacking. In addition, it has recently been demonstrated that in tri- and tetraploid oocytes of Bombyx mori (42, and unpublished observations) correction or modification of chromosome pairing takes place by dissolvment and reassembly of synaptonemal complexes. The reorganizations of trivalents into bivalents and univalents as well as that of quadrivalents into bivalents clearly show that the formation of synaptonemal complexes is followed by a turnover of the complexes which tends to optimize a pairing in the form of bivalents. This supports the idea that also interlockings may be corrected by dissolution and reassembly of synaptonemal complexes in organisms where crossing over is lacking or at stages prior to crossing over.

This assumption gains further support by the fact that in tetraploid Bombyx males where crossing over takes place reorganization of quadrivalents into bivalents is rare or absent. Light microscopical analysis of tetraploid spermatocyte nuclei at metaphase I has demonstrated that less than the expected $56(4 n / 2)$ bivalents are present in the metaphase plate (46). By analogy, correction of interlockings by disintegration and reformation of synaptonemal complexes may not be possible if the complex is stabilized by crossovers in the segments between the nuclear envelope and the site of the interlocking.

The dynamic nature of pairing has also been demonstrated in human spermatocytes. In a translocation heterozygote, a quadrivalent is invariably present at early pachytene while at mid pachytene, two separate bivalents were found in one of the four reconstructed nuclei (22). It is therefore possible that some of the interlockings in human spermatocytes are resolved by dissolution and reformation of synaptonemal complexes. The majority of interlockings in human spermatocytes appear, however, to be resolved by breakage and rejoining of chromosomes which may suggest that crossing over has occurred in the paired segments of the interlocking bivalents. In this context it is of interest that recombination nodules are associated with the synaptonemal complex already at early zygotene.

Although general conclusions cannot at present be made concerning the frequency of interlockings in other organisms, the present study and the investigations on Bombyx (40) do suggest that chromosome pairing a zygotene does not proceed as unimpeded as commonly believed. Likewise, it is not possible to evaluate the extent of chromosome and bivalent breakage at late zygotene in other organisms. If breaks are regular features of the zygotene chromosome pairing it is conceivable that 
breakage and rejoining is confined to specific regions of the DNA.

It has been proposed by $\operatorname{KING}(25,26)$ in his synaptomere-zygosome theory for synaptonemal complex formation that the lateral components of the complex are composed of transcriptionally inactive polynucleotide segments the synaptomeres - which are scattered along each chromatid and which by folding of the DNA come into proximity thereby establishing the continuity of the lateral components. It is furthermore assumed that corresponding synaptomeres from the two sister chromatids of each chromosome are associated side by side. A similar model for lateral component organization has been suggested by vON WETTSTEIN (50) although he assumes that corresponding DNA segments of the two sister chromatids are incorporated into the lateral components alternately. These models provide a simple explanation for the sometimes overlooked but important feature of homologous chromosomes, namely that they are always of the same length whether they are completely paired with a synaptonemal complex, present as partially paired bivalents, or even as univalents $(19,42,53)$. The internuclear variation in the length of a given chromosome which is common in most organisms $(3,12,15,20,40,53)$ is thus not reflected by a comparable variation in the intranuclear lengths of the two homologues.

If this concept of lateral component organization is correct, the resolution of interlockings (as well as untangling of intertwined chromosomes) by breakage and rejoining of chromosomes or bivalents would be greatly facilitated if the breakage of lateral components occureed in the polynucleotide segments (the synaptomeres) of both sister chromatids leaving the long DNA loops outside the lateral component intact. The breakage may either be a simple break caused by the physical tension created by chromosome movements during the pairing or be an enzymatically controlled process induced when two nonhomologous lateral components are pulled into close proximity. In those nuclei where more than one break have occurred the formation of chromosome rearrangements would be facilitated if rejoining of fragments from different chromosomes is possible.

\subsection{Recombination nodules \\ 4.2.1. Morphology}

In human spermatocytes recombination nodules exhibit substantial variation in size, shape and density, as well as in their position with respect to the central region of the synaptonemal complex. The variation is continuous and a classification into well-defined categories is not possible. It is the impression, however, that nodules at early pachytene are larger, denser and more frequently positioned adjacent to the central region of the complex than those of early zygotene nuclei. Comparable intranuclear variation in the morphology of the nodules has been described in Ascomycetes and Basidiomycetes $(3,15,53)$. In Neurospora, for example, the length of the nodules ranges from 55 to $210 \mathrm{~nm}$ and the width from 20 to $90 \mathrm{~nm}$. The length and width exhibit a very low linear correlation and the distributions of length and width are nearly normal distributions although an indication of bimodality in the length distribution was noted (15). In Drosophila, most recombination nodules are roughly spherical with diameters ranging from 40 to $180 \mathrm{~nm}$, the smaller ones being present in the central region, while the larger ones were reported to be located adjacent to the complex (4). In a personal communication to GILLIES (15) CARPENTER recently reported that also ellipsoidal nodules are present at early pachytene in Drosophila. Similar size differences have been reported in Chlamydomonas (45), where smaller nodules resembling those of fungi were common, while only two large nodules of the Drosophila type were present in each nucleus. In rat spermatocytes which also contain two types of nodules, the smaller ones were located in the central region, while the more frequent, larger ones were positioned adjacent to the central region (33).

\subsubsection{Number of recombination nodules}

The present study has demonstrated that recombination nodules are associated with the central region of the synaptonemal complex already at early zygotene. The number of nodules increases during zygotene reaching a maximum at late zygotene. Between late zygotene and early pachytene a $50 \%$ reduction of the number 
of nodules occurs followed by a complete (or almost complete) loss of nodules at mid pachytene (20). Upon reexamination of one early pachytene nucleus previously investigated (20) (with complete pairing af all bivalents and without a chromosome bouquet) 21 nodules were revealed. In the remaining 21 mid-late pachytene nuclei 5-10 nodule-like structures were discerned in each nucleus upon reexamination.

The presence of recombination nodules during zygotene has been found in Sordaria (53), Neurospora (15) and in Schizophyllum (unpublished observations). In the two ascomycetes the number of nodules remained constant from mid zygotene to diplotene where nodules were present in the retained fragments of the synaptonemal complex. In Schizophyllum, the stage dependent variation in the number of nodules in comparable to that revealed in human spermatocytes. In nuclei with nearly complete pairing assumed to be at the zygotene-pachytene transition 22 nodules were present per nucleus while at later stages only 13 nodules were found per nucleus (3). Also in Drosophila nodules appear to be transient structures, but the maximum number of spherical nodules was present at mid pachytene (4). In rat, MOENS (33) reported that nodules were only present in pachytene nuclei at stage VII of the seminiferous epithelium cycle while earlier pachytene stages lacked well-defined nodules. Finally, KUNDU and BOGDANOV (27) reported the presence of nodules in retained fragments of the synaptonemal complex at diplotene in Ascaris.

As mentioned previously, the original proposal by CARPENTER (4), that recombination nodules are somehow involved in recombination of linked genes, is supported by the correlation between the number and distribution of these structures and the frequency and distribution of crossing over in Drosophila (4), yeast (1) and Neurospora (15). Likewise, ZICKLER (53) demonstrated a high correlation between the number of nodules and the number of chiasmata.

The results obtained in the present study are more complex, and it is not possible to correlate the results with genetic data since the precise length of the genetic map in the human male is unknown. Only information on the number and distribution of chiasmata at diakinesis is available. HULTEN (24) reported an average of 50 chiasmata per diakinesis nucleus and showed that distal and terminal chiasmata were much more frequent than medial ones among nonacrocentric bivalents, the latter predominantly possessing proximal chiasmata. She also demonstrated a striking similarity between chiasma distributions in bivalents with the same number of chiasmata. Finally, it was noted that the short arms of the acrocentric bivalents were virtually devoid of chiasmata while a chiasma was present between the sex chromosomes in $63 \%$ of the nuclei. The discrepancy between the mean number of nodules (75) at early pachytene and the mean number of chiasmata (50) may be attributed to the inherent difficulties in the light microscopical analysis to resolve two or more closely positioned chiasmata or it may be due to a terminalization of chiasmata. The observation that terminal chiasmata are frequent at diakinesis while the telomere region at early pachytene is only slightly enriched in nodules is in support of the latter possibility. If terminalization occurs any comparisons of the distributions of nodules and chiasmata will be biased, the degree being dependent upon the extent of terminalization. However, the number of chiasmata and the number of nodules in the $\mathrm{XY}$ bivalent exhibit a fairly good correlation, $27 \%$ of the early pachytene sex bivalents being devoid of nodules compared to $37 \%$ precociously separated $\mathrm{X}$ and $\mathrm{Y}$ chromosomes (thereby indicating the absence of a chiasma) (24).

As in other organisms, the ultrastructural counterpart of chiasmata in human spermatocytes appears to be short stretches of synaptonemal complex retained between the homologous chromosomes. The diplotene stage has not yet been analyzed but reconstructions of metaphase I bivalents have revealed such fragments embedded in the condensed chromatin of the bivalents (21). These fragments were all devoid of recombination nodules. It thus appears that the elimination of recombination nodules at early-mid pachytene is permanent. The very long duration of the pachytene stage in human males amounting to 16 days (17) may ac- 
count for this precocious disappearance of recombination nodules. If the nodules present at early pachytene are related to crossing over, two explanations for their subsequent disappearance may be put forward. If the nodules contain the enzymatic and structural machinery for recombination, degradation of nodules may occur immediately after the recombination event is completed. Alternatively, it may be hypothesized that the homologous DNA regions which have undergone crossover or gene conversion, together with the associated nodule, move from the central region into the surrounding chromatin during early-mid pachytene.

If recombination nodules are involved in genetic recombination (with or without exchange of flanking markers) it is possible that they contain the enzymatic and structural machinery required for recombination. A likely constituent of the nodules is a protein comparable to the gene 32 protein of bacteriophage T4. This protein with its DNA binding capacity and affinity for membranes has been shown by Mosig, BERgQVIST and Bock (37) to interact with DNA polymerase, ligase and recombination nucleases thereby facilitating the coordinated action of these enzymes. Proteins, similar to the gene 32 protein, have been isolated from lipoprotein fractions of Lilium microsporocytes and mammalian spermatocyte nuclei by HoTTA and STERN, who in a number of biochemical experiments also have demonstrated the presence of the enzymes required for DNA nicking and repair during meiotic prophase (44). In this context it is of interest that FIIL and MoENS (8) proposed that the synaptonemal complex may have properties in common with the nuclear membrane and nuclear pores.

It has been demonstrated in several ascomycetes and in Drosophila that about $50 \%$ of the gene conversion events are associated with exchange of flanking markers. It is considered that both reciprocal and nonreciprocal recombination events are generated by a unitary mechanism (18, 34). In Schizophyllum (3) and in human spermatocytes the number of nodules is reduced by $50 \%$ from late zygotene to early pachytene. If this decrease is related to the $1: 1$ ratio of nonreciprocal to reciprocal recombi- nation events, it can be imagined that only those nodules which participate in a crossover remain associated with the synaptonemal complex until the end of early pachytene. It is, however, also possible that the observed decrease may be attributed to fusion of juxtapositioned nodules as indicated by the increase in size of the nodules in human spermatocytes and by the observation that many nodules at early pachytene are dumbbell shaped.

The study of 21 early pachytene nuclei has revealed 19 autosomal bivalents and 6 XY bivalents without nodules. If this also implies the absence of crossing over in these bivalents nondisjunction is likely to occur at anaphase I. Lack of recombination nodules is especially prevalent among the shorter bivalents and in particular in the acrocentrics and the $X Y$ bivalent. Therefore, first division nondisjunction in human spermatocytes would be expected to involve such chromosomes more frequently.

\subsubsection{Distribution of recombination nodules}

The analysis of the nodule distribution along the bivalents and its possible correlation with that expected from genetic data are in all cases based on the assumption that the nodules remain fixed (i.e., are unable to move along the synaptonemal complex) once associated with the central region. The same assumption is made without direct evidence in the following discussion.

The mean number of nodules per unit length of the bivalents was found in the present study to be similar throughout the bivalents. The centromere region, the secondary constrictions, and the short arms of the acrocentric bivalents had lower numbers of nodules than expected from a random distribution. The telomere regions and the $X Y$ bivalent had nodules in excess. A low number of nodules in centromere regions has also been reported in Drosophila (4), in Schizophyllum (3) and in Neurospora (15). In the latter case, the position of the centromere could not be established directly but was estimated by comparison with mitotic chromosomes. At early pachytene the telomere region in Neurospora is only slightly enriched in nodules in contrast to Sordaria where ZICKLER (53) has demonstrated the presence of a nodule 
in at least one arm of each bivalent $0.5-1.0 \mu \mathrm{m}$ from the telomere.

It is thus evident that recombination nodules are not positioned entirely at random along the bivalents and species specific differences are apparent. This raises the question as to whether the deviation from randomness in the result of differences in affinity between nodules and the individual regions of the bivalents or whether the nonrandom distribution is generated by the combined effects of differences in the rate of chromosome pairing and synaptonemal complex formation, and variations in the number of nodules available during zygotene.

In human spermatocytes, structures resembling recombination nodules are occasionally seen during zygotene either associated with unpaired lateral components, with precursor material for the central region, or (in one case only, see Figure 20) with a short synaptonemal complex fragment between nonhomologously paired chromosome regions. An association between recombination nodules and precursor material for central regions has been described in Neottiella (51) and provides an unequivocal example of a nonspecific binding of a recombination nodule to the central region of the complex. The present observations clearly demonstrate that recombination nodules may be incorporated into the central region during the formation of the synaptonemal complex, i.e., in the pairing fork, although nodules also associate with a completed complex as illustrated by the decrease in synaptonemal complex length per nodule from early to late zygotene.

The observations described above substantiate the possibility that the distribution of nodules at late zygotene primarily is a function of the intranuclear rate of synaptonemal complex formation rather than of differential binding capacity of individual regions of the bivalents. The delayed pairing of the short arms of acrocentric bivalents and of the secondary constrictions may thus account for the lack of nodules in these regions.

\subsubsection{Nodule-nodule distances and interference}

The present study has demonstrated that the $50 \%$ reduction of the number of nodules from late zygotene to early pachytene is accom- panied by a significant change in the distribution of distances between nodules. At late zygotene nodules are more frequently clustered than would be expected on the basis of a random distribution, while at early pachytene the reverse pattern is observed.

In Drosophila, CARPENTER (4) argued that the distribution of recombination nodules was compatible with the phenomenon of positive interference. A similar analysis in human spermatocytes is more difficult due to the fact that the physical distances over which interference may operate are unknown. Furthermore, the possibility that the nodules may also be involved in gene conversion without exchange of flanking markers introduces another parameter in the analysis of the distribution of nodule-nodule distances. It has been shown in several organisms $(18,34)$ that only crossovers show positive interference, while gene conversions (without flanking marker exchange) in adjacent intervals, as well as crossovers and gene conversions, occur independently.

It is likely that the number and distribution of recombination nodules at zygotene, and the change in these parameters from zygotene to pachytene, are somehow causally related to crossover interference, but additional work is obviously required to elucidate the complex interlationships between recombination nodules, crossing over, gene conversion and crossover interference.

\section{ACKNOWLEDGEMENTS}

We are indebted to Prof. D. voN WetTSTEIN for his encouragement and many helpful discussions during the course of the work and for his valuable criticisms of the manuscript. It is a great pleasure to thank JEAN SAGE for her skilled assistance in sectioning the large amount of material used in this study and for photographing several of the series. The outstanding technical assistance of ANN-SOFI STEINHOLZ, NinA Rasmussen, LeNa KongSRUd and INGE SOMMER is gratefully acknowledged. Finally, we thank cand. stat. L. SEJERSEN for assistance in the statistical analysis of the results. The work was supported by grant 202-76-1 BIO DK from the Commission of the European Communities. 


\section{REFERENCES}

1. BeYERS, B. \& L. GoETSCH: Electron microscopic observations on the meiotic karyotype of diploid and tetraploid Saccharomyces cereviciae. Proc. Nat. Acad. Sci. USA 72, 5056-5060 (1975)

2. BuUs, M. E. \& S. A. Henderson: Induced bivalent interlocking and the course of meiotic chromosome synapsis. Nature New Biol. 234, 243-246 (1971)

3. Carmi, P., P. B. Holm, Y. Koltin, S. W. RasmusSEN, J. SAGE, \& D. ZCKLLR: The pachytene karyotype of Schizophyllum commune analyzed by three dimensional reconstructions of synaptonemal complexes. Carlsberg Res. Commun. 43, $117-132$ (1978)

4 CARPENTER, A. T. C.: Electron microscopy of meiosis in Drosophila melanogaster females: II: The recombination nodule - a recombination associated structure at pachytene? Proc. Nat. Acad. Sci. USA 72, 3186-3189 (1975)

5. Darlington, C. D.: Recent advances in cytology. 2nd ed. Churchill, London $671 \mathrm{pp}$ (1937)

6. Dover, G. A. \& R. Ruley: The effect of spindle inhibitors applied before meiosis on meiotic chromosome pairing. J. Cell. Sci. 12, 143-161 (1973)

7. Feldman, M.: The effect of chromosomes 5B, 5D, and 5A on chromosome pairing in Triticum aestivum. Proc. Nat. Acad. Sci. USA 55, 14471453 (1966)

8. FilL, A. \& P. B. MoEns: The development, structure and function of modified synaptonemal complexes in mosquito oocytes. Chromosoma (Berl.) 36, 119-130 (1972)

9. Gairdner, A. E. \& C. D. Darlington: Ring-formation in diploid and polyploid Campanula persicifolia. Genetica 13, 113-150 (1931)

10. GASSNER, G.: Synaptinemal complexes in the achiasmatic spermatogenesis of Bolbe nigra Giglio-Tos (Mantoidea). Chromosoma (Berl.) 26, 22-34 (1969)

11. GILLIES, C. B.: Reconstruction of the Neurospora crassa pachytene karyotype from serial sections of synaptonemal complexes. Chromosoma (Berl.) 36, 119-130(1972)

12. Gillies, C. B.: Ultrastructural analysis of maize pachytene karyotype by three dimensional reconstructions of the synaptonemal complexes. Chromosoma (Berl.) 43, 145-176 (1973)

13. Gillies, C. B.: Synaptonemal complex and chromosome structure. Ann. Rev. Genet. 9, 91-109 (1975)

14. Gillies, C. B.: An ultrastructural analysis of chromosomal pairing in maize. Compt. Rend. Trav. Lab. Carlsberg 40, 135-161 (1975)
15. Gillies, C. B.: The relationship between synaptonemal complexes, recombination nodules and crossing over in Neurospora crassa bivalents and translocation quadrivalents. Genetics, in press

16. Gosden, J. R., A. R. Mitchell, R. A. BuckLAND, R. P. Clayton \& H. J. EVANS: The location of four human satellite DNAs on human chromosomes. Exptl. Cell Res. 92, 148-158 (1975)

17. Heller, C. G. \& Y. Clermont: Kinetics of the germinal epithelium in man. Recent. Prog. Horm. Res. 20, 545-575 (1964)

18. Holliday, R.: Recombination and meiosis. Phil. Trans. R. Soc. London B 277, 359-370 (1977)

19. Holm, P. B.: Three-dimensional reconstruction of chromosome pairing during the zygotene stage of meiosis in Lilium longiflorum (Thunb.) Carlsberg Res. Commun. 42, 103-151 (1977)

20. Holm, P. B. \& S. W. Rasmussen: Human meiosis I. The human pachytene karyotype analyzed by three dimensional reconstructions of the synaptonemal complex. Carlsberg Res. Commun. 42, 283-323 (1977)

21. Holm, P. B. \& S. W. RasmusSen: Three-dimensional reconstructions of meiotic chromosomes in human spermatocytes. In: A. de la Chapelle \& M. Sorsa eds., Chromosomes Today 6, 83-93 (1977)

22. Holm, P. B. \& S. W. Rasmussen: Human meiosis III. Electron microscopical analysis of chromosome pairing in an individual with a balanced translocation 46, XY, $t(5 p-; 22 p+)$. Carlsberg Res. Commun. 43---(1978)

23. HOTTA, Y. \& H. STERN: Absence of satellite DNA synthesis during meiotic prophase in mouse and human spermatocytes. Chromosoma (Berl.) in press

24. Hultén, M.: Chiasma distribution at diakinesis in the normal human male. Hereditas 76, 55-78 (1974)

25. KING, R. C.: The synaptomere-zygosome theory of synaptonemal complex formation. Amer. Zool. 8, 822 (1968)

26. KJNG, R. C.: The meiotic behaviour of the Drosophila oocyte. Int. Rev. Cyt. 28, 125-168 (1970)

27. Kundu, S. C. \& Yu. F. Bogdanov: Ultrastructural studies of late meiotic prophase nuclei of spermatocytes in Ascaris suum. Chromosoma (Berl.) in press

28. LU, B. C. \& N. B. RadU: Meiosis in Coprinus II. Chromosome pairing and the lampbrush diplotene stage of meiotic prophase. Chromosoma (Berl.) 29, 305-316 (1970)

29. Maguire, M. P.: A new model for homologous chromosome pairing. Caryologia 27, 349-357 (1974) 
30. MAguire, M. P.: Homologous chromosome pairing. Phil. Trans. R. Soc. London B 277, 245-258 (1977)

31. MAther, K.: Meiosis in Lilium. Cytologia 6, 354-380 (1935)

32. MoEns, P. B.: Quantitative electron microscopy of chromosome organization at meiotic prophase. Cold. Spr. Harb. Symp. quant. Biol. 38, 99-107 (1973)

33. MoEns, P. B.: Structure and distribution of synaptonemal complex crossover nodes in rat spermatocytes. Can. J. Genet. Cytol, in press

34. Mortimer, R. K. \& S. Fogel: Genetical interference and gene conversion. In: Mechanisms in Recombination. R. F. Grell, ed., Plenum Press New York \& London, 263-275 (1974)

35. Moses, M. J.: Synaptinemal complex. Ann. Rev. Genet. 2, 363-412 (1968)

36. Moses, M. J.: Synaptonemal complex karyotyping in spermatocytes of the Chinese hamster (Cricetulus griseus). II. Morphology of the XY pair in spread preparations. Chromosoma (Berl.) 60, 127-137 (1977)

37. Mosig, G., W. Bergquist, \& S. Bock: Multiple interactions of a DNA-binding protein in vivo. III. Phage T4 gene-32 mutations differentially affect insertion-type recombination and membrane properties. Genetics 86, 5-23 (1977)

38. Niebuhr, E.: Cytologic observations in 35 individuals with a $5 p^{-}$karyotype. Hum. Genet. 42 , 143-156 (1978)

39. Paris Conference (1971): Standardization in human cytogenetics. Cytogenetics 11, 313-362 (1972)

40. RASMUSSEN, S. W.: The meiotic prophase in Bombyx mori females analyzed by three-dimensional reconstructions of synaptonemal complexes. Chromosoma (Berl.) 54, 245-293 (1976)

41. RASMUSSEN, $S$. W.: The transformation of the synaptonemal complex into the "elimination chromatin" of Bombyx mori oocytes. Chromosoma (Berl.) 60, 205-221 (1977)
42. Rasmussen, S. W.: Chromosome pairing in triploid females of Bombyx mori analyzed by three dimensional reconstructions of synaptonemal complexes. Carlsberg Res. Commun. 42, 163-197 (1977)

43. RILEY, R. \& C. N. LAW: Genetic variation in chromosome pairing. Adv. Genet. 13, 57-114 (1965)

44. Stern, H. \& Y. HotTA: Regulatory mechanisms in meiotic crossing over. Ann. Rev. Plant Physiol. $29,415-436$ (1978)

45. StoRms, R. \& P. J. HASTINGs: A fine structure analysis of meiotic pairing in Chlamydomonas reinhardi. Exptl. Cell Res. 104, 39-46 (1977)

46. TAZIMA, Y.: The genetics of the silkworm. Logos Press \& Academic Press, London 253 pp (1964)

47. TRES, L. L.: Extensive pairing of the XY bivalent in mouse spermatocytes as visualized by wholemount electron microscopy. J. Cell Sci. 25, 1-15 (1977)

48. WALTERS, M. S.: Evidence on the time of chromosome pairing from the preleptotene spiral stage in Lilium longiflorum "Croft". Chromosoma (Berl.) 29, 375-418 (1970)

49. WestergaARD, M. \& D. von Wettstein: Studies on the mechanism of crossing-over IV. The molecular organization of the synaptinemal complex in Neottiella (Cooke) Saccardo (Ascomycetes). Compt. Rend. Trav. Lab. Carlsberg 37, 239-268 (1970)

50. WetTstein, D. vON: The synaptinemal complex and four-strand crossing over. Proc. Nat. Acad. Sci. USA 68, 851-855 (1971)

51. WestergaArd, M. \& D. von Wettstein: The synaptonemal complex. Ann. Rev. Genet. 6, 71-110(1972)

52. WetTSTEIN, D. von: The assembly of the synaptinemal complex. Phil. Trans. R. Soc. London B 277, 235-243 (1977)

53. ZiCKLER, D.: Development of the synaptonemal complex and the "recombination nodules" during meiotic prophase in the seven different bivalents of the fungus Sordaria macrospora Auersw. Chromosoma (Berl.) 61, 289-316 (1977) 